UC-700

Issued: April 1995

Authentication of Reprocessing Plant

Safeguards Data through Correlation Analysis

Tom L. Burr

Lawrence E. Wangen

Mark F. Mullen 



\section{DISCLAIMER}

This report was prepared as an account of work sponsored by an agency of the United States Government. Neither the United States Government nor any agency thereof, nor any of their employees, make any warranty, express or implied, or assumes any legal liability or responsibility for the accuracy, completeness, or usefulness of any information, apparatus, product, or process disclosed, or represents that its use would not infringe privately owned rights. Reference herein to any specific commercial product, process, or service by trade name, trademark, manufacturer, or otherwise does not necessarily constitute or imply its endorsement, recommendation, or favoring by the United States Government or any agency thereof. The views and opinions of authors expressed herein do not necessarily state or reflect those of the United States Government or any agency thereof. 


\section{DISCLAIMER}

Portions of this document may be illegible in electronic image products. Images are produced from the best available original document. 


\section{CONTENTS}

\section{CHAPTER 1. INTRODUCTION, SUMMARY, AND RECOMMENDATIONS}

I. OVERVIEW OF METHODS CONSIDERED ........................................2

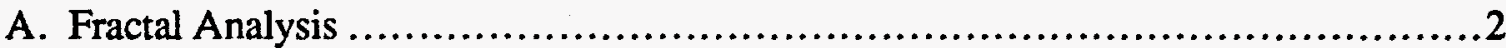

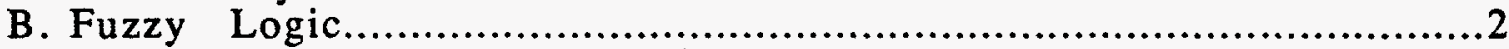

C. Process Fault Detection and Diagnosis Methods ........................................ 3

D. Nonlinear Time Series Analysis.............................................. 4

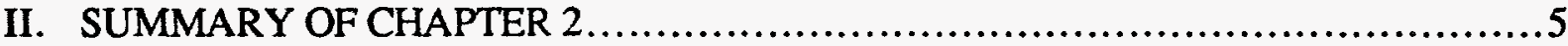

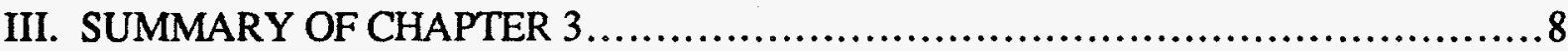

IV. CONCLUSIONS AND RECOMMENDATIONS...........................................

A. Conclusions Regarding Fault Detection ...........................................9

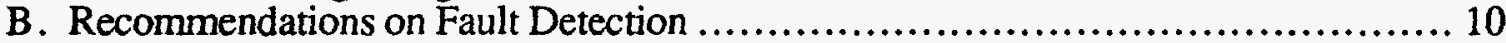

C. Conclusions Regarding Nonlinear Time Series Analysis ......................... 10

D. Recommendations Regarding Nonlinear Time Series Analysis .................. 10

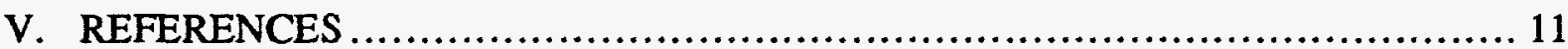

\section{CHAPTER 2. ON FAULT DETECTION AND DIAGNOSIS AND ITS POTENTIAL APPLICABILITY TO SAFEGUARDS IN THE NUCLEAR FUEL CYCLE}

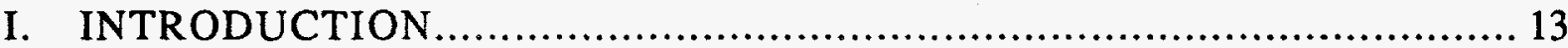

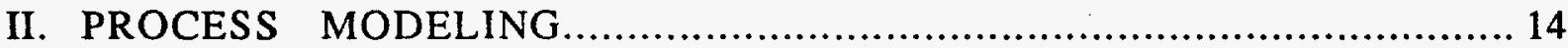

III. GENERATION OF RESIDUALS FOR FAULT DETECTION.............................. 15

A. Model-based Fault Detection....................................................... 16

1. Observer Methods .................................................................. 17

2. Parameter Identification Methods.......................................... 17

3. Parity Space Approach .................................................. 18

B. Measurement-based Fault Detection ........................................ 18

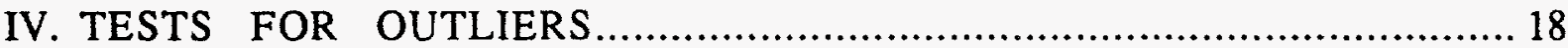

A. Outlier Tests Based on the Gaussian Distribution................................. 19

B. Some Results from the Fault Detection Literature ................................ 20

C. Some Multivariate Outlier Detection Methods and Statistical Literature ............... 24

1. Outliers Using Principal Components Analysis......................................... 25

2. Literature Example from a Reprocessing Plant......................................... 27

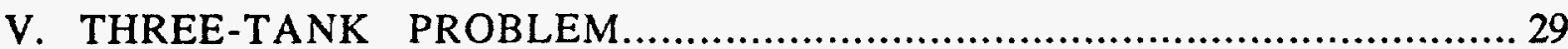

A. The Three-Tank Model .................................................................... 29

B. Model Predictions and Uncertainty Under Normal (No Fault) Operations............... 32

C. Diversion Scenarios................................................................. 35

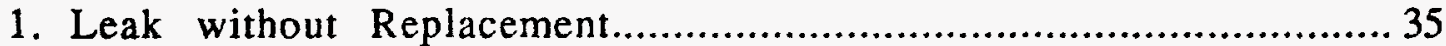

2. Leak with Replacement ................................................... 39

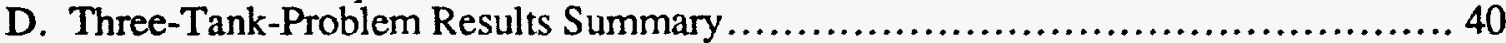

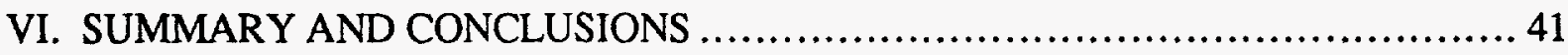

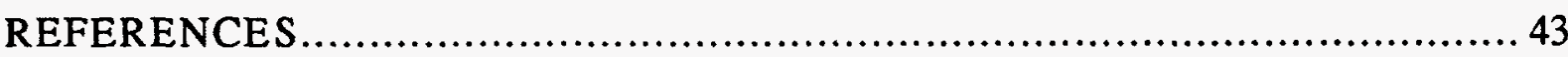




\section{CONTENTS (cont.)}

CHAPTER 3. NONLINEAR TIME SERIES ANALYSIS APPLIED TO ANOMALY DETECTION IN NEAR-REAL-TIME ACCOUNTING DATA

I. INTRODUCTION. 45

II. NONLINEAR TIME SERIES 49

III. TRADITIONAL METHODS. 56

IV. DATA ANALYSIS ....................................................... 58

V. ADDITIONAL ESTIMATION PROCEDURES........................................ 76

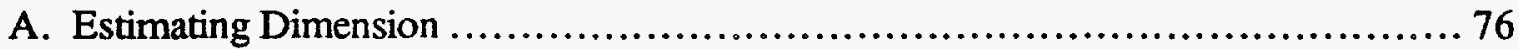

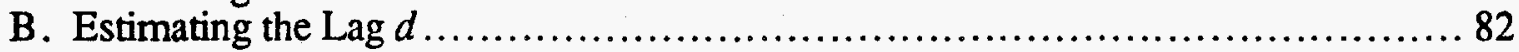

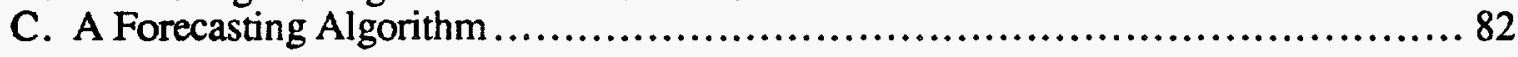

VI. COMPARISON OF NONLINEAR AND LINEAR PREDICTION RESULTS.......... 83

VII. SUMMARY AND PLANS FOR FUTURE RESEARCH...................... 88

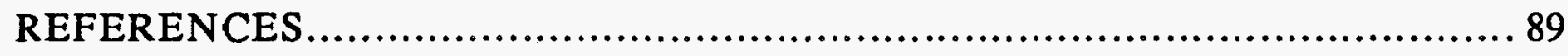




\title{
AUTHENTICATION OF REPROCESSING PLANT SAFEGUARDS DATA THROUGH CORRELATION ANALYSIS
}

\author{
by
}

Tom L. Burr, Lawrence E. Wangen, and Mark F. Mullen

\begin{abstract}
This report investigates the feasibility and benefits of two new approaches to the analysis of safeguards data from reprocessing plants. Both approaches involve some level of plant modeling. All models involve some form of mass balance, either applied in the usual way that leads to material balances for individual process vessels at discrete times or applied by accounting for pipe flow rates that leads to material balances for individual process vessels at continuous times. In the first case, material balances are computed after each tank-to-tank transfer. In the second case, material balances can be computed at any desired time. The two approaches can be described as follows. The first approach considers the application of a new multivariate sequential test. The test statistic is a scalar, but the monitored residual is a vector. The second approach considers the application of recent nonlinear time series methods for the purpose of empirically building a model for the expected magnitude of a material balance or other scalar variable. Although the report restricts attention to monitoring scalar time series, the methodology can be extended to vector time series.
\end{abstract}




\section{CHAPTER 1}

\section{INTRODUCTION, SUMMARY, AND RECOMMENDATIONS}

The objective of Task D.89 was to investigate the feasibility and potential benefits of a variety of new approaches to the analysis of safeguards data from reprocessing plants. The intention was to identify one or more methods that might be used to facilitate safeguards by taking advantage of inherent correlations or relationships between different subsets of the data.

Among the methods initially identified as potential candidates for investigation were fuzzy logic, fractal analysis, expert systems, the theory of zero knowledge proofs, and process fault detection and diagnosis. Following our initial literature study, nonlinear time series analysis was added for consideration.

For process control and other reasons, there is a strong trend towards increasing automation of chemical processing plants, including reprocessing plants. As a part of this trend, the quantity of data potentially available for safeguards will be far greater in future reprocessing plants than in current plants. This data consists of control data and physical and chemical measurements of process inputs and outputs during plant operations. This data also includes traditional inventories and transfers of nuclear materials. Not only will more parameters be monitored, but data collection will be more frequent than in the past. Although it has not been decided if this process data will be available to the International Atomic Energy Agency (IAEA), the IAEA might be able to use it to assist with safeguards, if workable methods of authenticating and analyzing the data can be developed.

The data authentication and analysis methods must be tailored to the characteristics of the facilities and the collected data. Safeguards data from future reprocessing plants may include IAEA measurements, operator accounting measurements, process control data, and data from other sources, such as fuel fabricator's data and reactor burnup data. Some of the operator data will be verified directly by the IAEA through independent measurements. Data that are not directly verified might be indirectly verified through the theoretical or empirical relationships (correlations) that exist between different data elements. For example, relationships will exist among concentration, density, acidity, and temperature; between volumes/weights transferred out of tank A and into tank B; and among fuel fabricator's data, burnup, and measured concentrations of plutonium and uranium.

The results of this assessment study are intended to assist the planning for implementation of safeguards in future large-scale reprocessing plants and to provide guidance regarding follow-up research and development efforts related to the use of safeguards data at these plants. In Sec. IV of Chapter 1, we will make some recommendations regarding follow-up efforts. 


\section{OVERVIEW OF METHODS CONSIDERED}

In the first part of this study, we reviewed the literature. We covered fractal analysis, fuzzy logic, process fault detection and diagnosis, and nonlinear time series analysis. Highlights of that literature review are summarized below.

\section{A. Fractal Analysis}

A portion of the literature on fractal analysis that might be applied to reprocessing plant safeguards data was reviewed. The key concept is that time series and fractals have a connection. Near-real-time process measurement and modeling in future reprocessing plants will produce many examples of time series, including lower-level process parameters as well as the more traditional material unaccounted for (MUF), adjusted running book inventory (ARBI), and other high-level indices. We expect many more observations in these time series than in safeguards time series that have been used. This abundance of data provides an opportunity to use a variety of time series analysis techniques that have not previously been applied to safeguards data. Fractal analysis is one such technique.

Our initial conclusion is that fractal analysis of time series is mainly a descriptive technique for providing a measure of the "fractal dimension" and other descriptors of the series. Changes in the fractal dimension could be used to detect changes in the correlation structure of a time series and, thus, might indicate certain types of anomalies of interest for safeguards. However, it is not clear that the fractal dimension or any of the various other measures suggested by fractal analysis will be an improvement to classical methods of time series analysis, such as the autocorrelation function, or alternatives proposed for safeguards applications, for example Page's test. The relative merits of some of these measures are discussed in Chapter 3.

Using fractal methods, a possible approach would be to apply fractal analysis to the noise remaining (residuals) after fitting some model to the original time series data rather than applying the method to the original data. The intention would be to use fractal analysis to help detect and classify structure (anomalous behavior not accounted for by the fitted model) in the residuals.

\section{B . Fuzzy Logic}

Fuzzy set theory provides a formalism for representing uncertainty and differs from the probabilistic methods commonly used in safeguards. Although there have been various claims about the usefulness and applicability of fuzzy logic, its major appeal seems to be its ability to handle "linguistic uncertainty" and "linguistic variables" in a fairly natural way. Linguistic uncertainty is the kind of uncertainty that is commonly described in words, for example, "small value of MUF," "fairly large value," "larger than normal value," and so forth. In this example, 
MUF would be a linguistic variable because its values are natural language words rather than the more conventional real numbers. One of the features of fuzzy set theory is that it provides recipes for performing calculations with linguistic variables.

In principle, there are many ways in which this idea could be applied to safeguards. However, for most of these potential applications, it is not clear whether fuzzy approaches have any real advantages when compared to more familiar safeguards tools. For example, if we are interested in analyzing MUF or a sequence of MUFs, the familiar quantitative approaches are undoubtedly superior because they use the available information more fully. As mentioned above, the comparative advantage of fuzzy logic seems to reside in its ability to capture expert knowledge, as expressed in terms of linguistic variables. At this point, the most interesting possibility seems to be anomaly detection (fuzzy pattern recognition or fuzzy classification). In this context, fuzzy set concepts may be applicable in conjunction with expert systems, neural networks, or process models.

Because process control is an inexact science, there has been considerable interest in the use of fuzzy methods for process control. Many of the widely advertised applications are in this category. This application may have value for the plant operator, but it is not clear how it could be used for NRTA and international safeguards where we are interested in detecting diversion rather than in controlling a process using inexact methods.

\section{Process Fault Detection and Diagnosis Methods}

Although somewhat removed from the traditional safeguards arena, the chemical industry, for many years, has been considering process control issues that are directly applicable to reprocessing plant safeguards. The chemical engineering literature contains a significant amount of recent work in the area of "process fault detection and diagnosis." The following quote from a 1990 survey paper ${ }^{1-1}$ indicates the relevance of this topic to the International Atomic Energy Agency's (IAEA's) safeguarding of nuclear materials at a future reprocessing plant:

Automatic control systems are becoming more and more complex ... . Consequently, there is a growing demand for fault tolerance which can be achieved not only by improving the individual reliability's of the functional units but also by an efficient fault detection, isolation and accommodation concept .... A fault is understood as any kind of malfunction in the actual dynamic system, the plant, that leads to an unacceptable anomaly in the overall system performance. Such malfunctions may occur either in the sensors (instruments), or actuators, or in the components of the process ... . Over the last two decades, the basic research on fault detection and isolation [FDI] has gained increasing consideration worldwide. This development was (and still is) mainly stimulated by the abovementioned trend of automation towards greater complexity and the corresponding growing demand for higher availability and security of control systems ... . In the course of this development a novel philosophy for the FDI 
methodology has emerged and is increasingly discussed. It is based on the use of analytical (i.e., functional) rather than physical redundancy. This implies that the inherent redundancy contained in the static and dynamic relationships among the system inputs and the measured outputs is exploited .... The appeal of the analytical redundancy approach lies in the fact that the existing redundancy can simply be evaluated by information processing under well-featured operating conditions (i.e., in the operation center) without the need of additive physical instrumentation in the plant. However, there is a price to pay for this benefit which arises from the need of the mathematical model....

Clearly, this is the same concept of inherent correlations in complex safeguards data that safeguards experts have discussed for many years, not only in the context of reprocessing plant safeguards but also in connection with the fuel cycle approach to safeguards mentioned in Paragraph 81 (c) of INFCIRC/153.1-2

As the preceding quotation indicates, a major difficulty in applying this methodology lies in formulating a mathematical model of the plant. At present, there is no guarantee that tractable, realistic models can be developed for a plant as complex as a large-scale reprocessing plant. Various approaches can be used to develop a mathematical model of a process. These include fundamental models based on a physical/chemical description of the process and empirical methods such as neural networks, pattern recognition, regression, and lumped-parameter differential equations. Knowledge-based models, i.e., expert systems, have also been proposed. The chemical engineering literature documents a variety of process engineering models that are potentially applicable within the context of reprocessing plant safeguards. Because others have been or are investigating neural network and expert system approaches, for this task we chose to focus our efforts on fault detection and diagnosis as discussed in the chemical engineering literature. Thus, Chapter 2 provides a detailed description of our investigations into the methods of fault detection and diagnosis.

\section{Nonlinear Time Series Analysis}

In addition to process fault detection and diagnosis, we decided to focus our effort on nonlinear time series analysis. This choice was based on two main considerations:

(1) nonlinear time series analysis is less developed than linear time series analysis, so it needs further investigation; and

(2) fractal analysis and fuzzy controller methods are subsets of nonlinear time series analysis, so the approach we take provides a general framework for analysis of any scalar time series.

The following sections include a summary of Chapter 2 and Chapter 3 followed by a brief section on our conclusions and recommendations. 


\section{SUMMARY OF CHAPTER 2}

A fault or abnormal condition occurs when some state of the process, e.g., temperature, pressure, or mass of plutonium, is outside acceptable limits. Thus, fault detection is a part of process control and is based on the availability of redundant process information. The methods consist of two general categories based on the kind of redundant information possessed about a process. Both categories assume the availability of measurement data. "Measurement-based" methods rely on redundant measurements. We include in measurement-based methods those using historical data as "target" values or set-points against which new measurements are compared. "Analytical-based" methods involve redundant information obtained from a mathematical or knowledge-based model (expert system) of the process. We illustrate the concept in which model estimates are compared with measurements to determine the presence or absence of a fault with some desired degree of confidence.

If a process is operating with no faults, we expect that all of the redundant information will be consistent except for the unavoidable presence of modeling and measurement errors. Fault detection methods are usually designed to estimate residuals, i.e., deviations between what is expected and what is observed, based on this redundant information. That is, measured values are compared with model predictions of the same variable. Observers compare modelbased estimates with measured observations of process states. Parameter identification methods refer to the on-line estimation of the mathematical parameters, which cannot be directly observed, of the process model and their subsequent evaluation to provide information about the present status of the process. A third method, the consistency (parity space) approach, uses a combination of measurements and consistency relationships, such as materials balance equations, chosen to provide a set of reliable comparisons. The traditional MUF is of this type.

Having compared the redundant data and obtained the residuals, the next problem in fault detection is determining when the residuals are "large" enough to indicate occurrence of a process fault. Thus, testing residuals, e.g., outlier testing in the statistical sense, is a major concern of fault detection and diagnosis. After a decision that a fault has occurred (fault detection), appropriate action for process control requires isolation of the location and determination of the cause of the fault, i.e., fault diagnosis, and finally some corrective action. For safeguards, appropriate action might be to investigate whether a diversion has occurred, if so, to determine where and how it occurred, and to identify appropriate follow-up action. Obviously fault diagnosis is more difficult than fault detection because it requires more detailed knowledge of the processes and more numerous and specific measurements. 
Many different outlier tests for fault detection have been proposed in the literature. They include maximum likelihood ratio, sequential likelihood ratio, Bayesian, and the univariate $z$ or student $t$ tests. Multivariate residuals with multinormal distributions can be individually evaluated by univariate tests, if appropriate, or by multivariate tests, such as the Mahalanobis distance. When a univariate test is applied to multiple variables, the critical values should be adjusted to account for the multiple tests, if the same overall significance level is desired. For example, if 10 uncorrelated variables are to be examined and an overall significance level of 5\% is desired, the critical value of $5 \%$ is divided by 10 so that each test has an alpha of $0.5 \%$. Several proposed tests are discussed in Chapter 2. The main distinctions are univariate versus multivariate and the use of an appropriate covariance matrix.

Often these calculations use estimates of the mean and covariance. If these estimates were obtained from data that contained faults, the outliers may be difficult to identify because they are "masked," that is, the estimates of mean and covariance are distorted by the outliers. Masking can be particularly severe when there are several unrelated outliers or faults in the sample data set. This problem motivates us to use robust estimates of the mean and covariance which are obtained in such a way as to negate the effect of the outliers. Using robust estimates, we can calculate robust distances that provide sensitive methods for detecting faults.

When the data sets are composed of many variables that are highly correlated, principal components (PC) analysis can be applied to data reduction and interpretation. The correlations provide redundancies that sometimes can be useful for detecting faults. Graphical identification of faults is a possibility when dimensionality reduction methods such as PC analysis are used. Several measures are available for detecting faults from a PC model of the data, which will be discussed in Chapter 2. In a recent paper, ${ }^{1-3}$ analytical data from spent nuclear fuel reprocessing in Germany was interpreted by principal components analysis. Sixteen variables, including a measure of burnup, plutonium and uranium isotope concentrations, and isotopic ratios were reported for ten different reprocessing batches. We have applied some of the fault detection tests to a PC analysis of these data. For these data 3 PCs explained $92 \%$ and 4 PCs explained $97 \%$ of the (normalized) variance. Neither normalized $z$-scores or Mahalanobis distances indicated any outliers for this data set, a conclusion in agreement with that of the publication.

We simulated a three-tank system containing nitric acid, plutonium, and uranium. The dynamics are described by a system of coupled differential equations based on total mass balances for each tank and on individual mass balances for each chemical species.

[Time rate of change of mass $]=[$ Mass in $]-[$ Mass out $]$. 
For given input flows, initial tank volumes and initial concentrations of nitric acid, plutonium and uranium, the differential equations are solved to give the outputs, i.e., the volumes and concentrations in the tanks at various times. The density of each tank solution is determined from empirical relationships between density and concentrations of nitric acid, plutonium, and uranium. These variables are the model predictions that are compared to "measured" values obtained by adding measurement uncertainty to the known correct values to give residuals that are tested for fault detection.

Two diversion scenarios were investigated at several different leak rates: 1) a steady leak from the second tank without replacement and 2) the same leak but the lost solution is replaced with water. In practice, the model would not know about the leak and thus would make erroneous predictions because it assumes normal operations. The true conditions, i.e., the loss because of leakage, are reflected in the measured data. Results of fault detection tests for the $0.5-\mathrm{L} / \mathrm{h}$ leak, which was easily detected, are summarized. The concentrations of plutonium and uranium and the density were detected as outliers in the second tank under the leak-with-waterreplacement scenario, whereas only volume was detected as a fault for the leak-withoutreplacement scenario. Replacing the removed volume with water diluted the concentrations enough to make a large difference in all concentration variables as well as in the density, which is based on concentrations. This demonstrates the value of measuring more variables for process fault detection. The Mahalanobis distances (a distance based on all variables taken together) are shown for three different leak rates. In all cases the values are larger, indicating more statistical significance, for the leak with replacement scenario. Only the $0.5-\mathrm{L} / \mathrm{h}$ rate is significant at the 5\% level using the Mahalanobis distances.

For the three-tank problem, univariate tests on individual variables and on individual principal components were equally effective at detecting losses of material. Because the principal components are linear combinations of individual variables, they might be expected to provide more sensitive detection of outliers or faults for situations where two or more correlated variables are affected by a fault. However, the correlations were not strong enough that we observed this effect in the present system. Multivariate tests based on the Mahalanobis distances were never as sensitive as the univariate tests, probably because this measure is diminished somewhat by those variables not affected by a leak. The leak-with-replacement scenario was detected with slightly more sensitivity than was the leak without replacement. This is undoubtedly because replacing the lost volume with water affects four variables (the density and the concentrations of nitric acid, plutonium, and uranium) rather than just one. 


\section{SUMMARY OF CHAPTER 3}

In Chapter 3 we consider univariate time series based on an assumption that long sequences of data (several hundred values) may be available in future automated reprocessing plants. The data could be MUF values, shipper-receiver differences between individual tanks, or other statistics arising from safeguards, process control, or measurement control. The most well-known analysis tool for univariate time series was popularized by Box and Jenkins in the 1970 s and is now known as Box-Jenkins time series analysis. This method of analysis is briefly reviewed in Chapter 3, where it is pointed out that Box-Jenkins analysis assumes that the expected value of each observation is a linear function of some subset of all previous observations and errors. We then introduce nonlinear analysis tools and compare them with linear Box-Jenkins methods for analyzing time series.

In recent years (see Chapter 3 references) there has been considerable interest in the curious behavior exhibited by certain nonlinear maps that produce random-appearing sequences or that produce unusual geometric objects known as fractals. We considered the applicability of this literature to safeguards. Because of the unavoidable presence of measurement errors, time series of interest to safeguards will always have a random, non-deterministic component. Unfortunately, the presence of this random component in the time series limits the usefulness of many of the deterministic techniques that have been suggested for analyzing nonlinear maps. Random error can completely change the behavior of a deterministic map. For example, the fractal dimension of the attractor (set of possible values after many iterations of the nonlinear map) of certain nonlinear maps is non-integral in the absence of random noise but is integral in the presence of random noise. We include test results of one method of estimating fractal dimension. Based on our limited experience, we do not recommend using estimated fractal dimension for safeguards.

Our current recommendation is to include techniques that can test for nonlinearity in a package of evaluation methods for time series. If tests do not indicate nonlinearity, there is no need to apply nonlinear estimation methods. If tests indicate nonlinearity, we recommend using nonlinear techniques for estimating the expected value of an observation in the time series sequence. Presumably, if a time series sequence fails tests for linearity, this expected value will be a nonlinear function of some subset of the previous observations.

In computer simulations we have experimented with several nonlinear estimation methods using both linear and nonlinear simulated data sets. An assumption of our present implementations is that the same functional dependence between an observation and some subset of the previous observations must hold throughout the entire time series. If this assumption is not valid, the time series must be divided into subsets where the assumption is satisfactory. This 
requires detailed knowledge of the process that is generating the sequence. Except for this potentially serious problem, the implementation of our procedures is straightforward.

Methods for estimating the number of previous observations that influence the present observations have also been implemented. In the linear time series literature, this number is called the lag, and in the nonlinear time series literature, it is called the dimension. We believe that empirical estimates of the lag may add to our understanding of the processes generating the data. In many cases, we have a good idea of a value for the lag. For example, in ordinary MUF sequences, a value of one is often a good first approximation for the lag because the ending inventory for $M U F_{j-1}$ is the beginning inventory for $M U F_{j}$. Therefore, if we ignore the effects of systematic errors, the lag is one. It should be informative to monitor estimates of the lag regardless of whether we think we know it.

Our main emphasis in Chapter 3 is to improve our ability to detect anomalies by using the best possible technique for predicting future values of the time series. By best possible, we mean that the standard error of residuals (difference between predicted and measured values) is minimized. This maximizes our sensitivity for detecting an anomaly, such as a loss of material. Our simulations suggest that, if the true functional form of the time sequence is linear, little or nothing is lost by applying nonlinear methods. However, if the true time series is nonlinear, large errors can result from using linear methods: indicating that nonlinear methods should be used. Thus, there may be a general advantage to using nonlinear methods.

Finally, nonlinear modeling may improve our understanding of processes generating the data through detection of unexpected functional dependencies. Thus, for reasons other than anomaly detection, we may wish to analyze time sequences containing many elements using nonlinear methods should they become available in the future.

\section{CONCLUSIONS AND RECOMMENDATIONS}

\section{A. Conclusions Regarding Fault Detection}

Developments in this field appear to offer significant benefits both for the plant operator and for the IAEA. The research is well thought out and rigorous and has a solid chemical engineering and statistical basis. It should have a positive impact on the future efficiency and safety of chemical processing plants. As with most theoretical $R \& D$, a scarcity of good examples of practical applications is the main limitation of the published work. Our understanding however, based on contacts associated with the chemical industry, is that many progressive industries are developing their own in-house, proprietary process control and fault detection methods.

It is most difficult to develop the mathematical model for implementation of model-based process fault detection for large reprocessing plants. This is despite the fact that many chemical 
systems can be modeled using either fundamental principles or empirical models. We believe that the most useful systems will involve a combination of qualitative (e.g., expert systems or fuzzy logic) and quantitative (mathematical models of unit processes) approaches. Simulation models, such as the facility simulation model, FACSIM, being developed at Los Alamos for the simulation of nuclear facilities, may have application as a modeling tool for fault detection.

\section{B . Recommendations on Fault Detection}

As mentioned above, a limitation of the published work on fault detection is a lack of demonstrated applications in operating plants. Using this methodology on data from an operating reprocessing plant would be a logical next step for this $R \& D$ and is our main recommendation. A joint project between researchers and operating engineers at a reprocessing facility would be desirable.

Another area of considerable interest is to further evaluate the three-tank system under different fault scenarios and to add more complicated unit processes, e.g., pulsed columns, to this system. We would attempt to use data from reprocessing to validate the approach.

Finally, we recommend that the utility of advanced multivariate time series be assessed. It is common to reduce multivariate time series to univariate time series by using simple linear combinations of the individual variables. However, more powerful and sophisticated methods exist and can be applied to the problem. It could be useful for safeguards to identify cases where analysis of time series as multivariate data is advantageous over performing the analysis on linear combinations of the univariate observations. Again, analysis of actual operating plant data should be part of such an assessment.

\section{Conclusions Regarding Nonlinear Time Series Analysis}

In our summary of Chapter 3 we recommended using tests for nonlinearity followed by the application of nonlinear estimation methods, if the tests for nonlinearity suggested a nonlinear time series. In theory, there should be little difficulty implementing the nonlinear methods. However, the assumption that a long sequence of observations is being generated by the same process is a practical difficulty. The presence of outliers caused by anomalous data or a shift in the operating conditions can unduly influence the nonlinear estimators and result in spurious alarms or anomalies. Problems of this nature are inherent in any attempt to use historical data to build rules for how we expect data from the process to behave.

\section{Recommendations Regarding Nonlinear Time Series Analysis}

In view of the emphasis in Chapter 3 , we have one main recommendation and a related recommendation. 
With respect to nonlinear time series analysis, our main recommendation is to apply the nonlinear testing and estimation techniques described in this report to actual safeguards data. The purpose would be to determine if the sensitivity to anomaly detection could be improved using the nonlinear methods. Long sequences, of length 200 or more, are needed for application of these methods. The data would be tested for nonlinearity, and, if nonlinearity were detected, we would apply the nonlinear estimation techniques to minimize the error variance of our estimates.

The second recommendation, associated with the main recommendation, is to use computer codes, developed for this project, to estimate and monitor both the correlation dimension and the lag of a time series. The correlation dimension is a qualitative tool that can be used to monitor changes in the behavior of a time series. The lag can be used as a quantitative tool for monitoring changes in the behavior of a time series. Based on knowing the process that generated the data, we expect to know the true lag for any time series of interest to safeguards. Therefore, if estimates of the lag suddenly change, or begin to disagree with the known lag, a change in the time series is indicated.

Finally, the nonlinear techniques are presently applicable only to univariate time series. Therefore, an additional recommendation is to investigate the use of a nonlinear analog of the cross-correlation function, used for multivariate time series, for multivariate safeguards data.

\section{REFERENCES}

1-1. Paul M. Frank, "Fault Diagnosis in Dynamic Systems Using Analytical and Knowledgebased Redundancy-A Survey and Some New Results," Automatica 26, 459-474 (1990).

1-2. "The Structure and Content of Agreements Between the Agency and States Required in Connection with the Treaty on the Non-Proliferation of Nuclear Weapons," International Atomic Energy Agency document INFCIRC/153 (June 1972).

1-3. S. Schoof, "Spent Nuclear Fuel Reprocessing: Chemometrical Treatment of Input Analytical Data," ESARDA Bulletin 18, 6-11 (1990). 


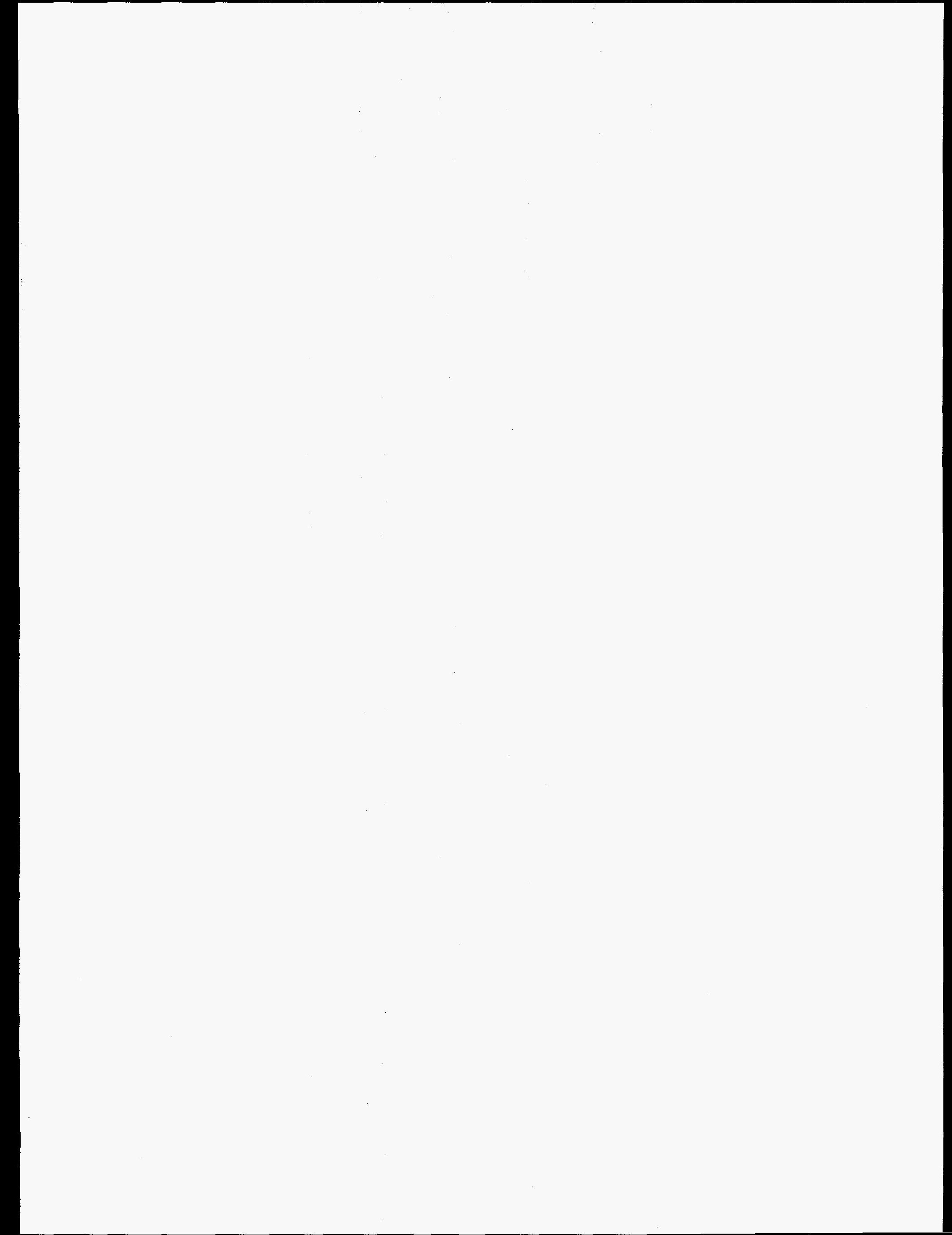




\section{CHAPTER 2}

\section{ON FAULT DETECTION AND DIAGNOSIS AND ITS POTENTIAL APPLICABILITY TO SAFEGUARDS IN THE NUCLEAR FUEL CYCLE}

\section{INTRODUCTION}

In recent years chemical processing plants have become more automated and there is little doubt that this trend will continue. Real-time process control for improved process efficiency and safety are foremost among various good reasons to automate. For automation and process control, timely information about the physical and chemical states of processes is needed. This information is used to detect and correct deviations from steady state that cause less than optimal operation and to alert personnel should unsafe conditions develop.

These factors have motivated the emergence of a new discipline in the automation and control field. Practitioners of this discipline are developing new and improved methods for the detection, isolation, and identification of deviations from nominal or desired process operating conditions. Such deviations may be called faults, disturbances, anomalies, or failures. The methods involve comparing data from process measurements with redundant information either from other process measurements or from process models to detect and identify faults so that appropriate action can be taken.

It is anticipated that these ongoing developments may provide opportunities for near-realtime accounting (NRTA) of nuclear materials. Diversion of special nuclear materials for unlawful purposes would be similar to a fault, such as a leak, in the above discussion. Within a materials balance area (MBA), in addition to process input data, sensor measurements will provide data on numerous variables, some of which may be redundant or correlated. Over many measurement cycles, a fairly large set of measurement data would be acquired. Under normal operating conditions this data is expected to have recognizable patterns that would change should a fault occur. Departure patterns would vary depending on the nature of the fault. Possibly materials diversion could be identified as specific types of fault patterns.

Thus, under the sponsorship of ISPO, Los Alamos National Laboratory is investigating the potential for using these data and fault detection methods for safeguards. Our purpose is to investigate methods of fault detection and diagnosis being developed and reported in the chemical processing literature and to evaluate their applicability to safeguards. Our focus is mainly on multivariate approaches that take advantage of data collected from multiple sensors. The work reported in this chapter was performed in two phases. The first phase was a literature review focusing on developments in fault detection and diagnosis from the chemical engineering 
literature and on the detection of multivariate outliers from the applied statistics literature. The literature review is also intended to provide a brief tutorial of the field to indicate the "state of the art." The second phase involved using a simulation model to predict process variables in a three-tank system for which measured values are also available (by simulation). Both predicted and measured values contain uncertainty. The differences between model-predicted and measured values, called residuals, are analyzed to provide statistical information under normal, nofault, operations. These can be contrasted with residuals observed when faults occur. The residuals from two different diversion scenarios are tested by univariate and multivariate fault detection and diagnosis methods.

In the following sections we provide a brief discussion of methods used to model processes, a review of methods used to generate the data tested for fault detection and diagnosis, and a discussion of some of the methods used to test these data to signal if a fault has occurred. In this chapter we are concerned with the analysis of multivariate data sets without regard to time series aspects.*

\section{PROCESS MODELING}

Fault detection and diagnosis depends on the evaluation of the consistency of redundant process information. Redundancy can be obtained either from replicate measurements of the same variables or from a process model that predicts measured variables. When redundancy is provided by a process model, it is necessary to develop a mathematical model of the process that can provide timely predictions based on process inputs. A diagnostic process model implies that we are interested in not only detecting a fault but also in determining its cause and, thus, enabling a solution. To be useful for diagnosis, a process model must contain knowledge about the fundamental characteristics of the process.

Three general approaches can be used separately or in combination to model processes for fault detection and diagnosis. These are theoretical models based on the fundamental relationships between process input and output variables, empirical models based on experimentally determined relationships between inputs and outputs, and knowledge models or expert systems based on systems of rules relating inputs and outputs.

Theoretical models are founded on the fundamental laws of conservation of mass, energy, and momentum, which are applied to definable subsystems of the process. For chemical systems these laws are usually expressed as systems of ordinary or partial differential

\footnotetext{
*The following chapter (III) investigates the potential for using nonlinear time series methods to treat sequences of univariate materials balance data for safeguards purposes.
} 
equations involving mass or energy balances for the subsystem. This approach is used to model the three-tank problem later in this chapter. Theoretical models are often augmented by empirical equations relating different variables of the system. For example, in the three-tank problem, an experimentally determined relationship between density and the concentrations of nitric acid, plutonium, and uranium is used to obtain a model prediction for density that can be compared to measured values for fault detection. Such relationships between different constituents of the system are called constitutive relationships.

Empirical models are based on the experimental determination of relationships between input and output data. This determination is called process identification in the chemical engineering literature and can be applied to any operating process. Developing the model involves actively determining outputs while varying inputs in a controlled manner to obtain a sufficiently large data set to cover normal operations or, alternatively, the more passive procedure of recording inputs and outputs over a sufficiently long time to obtain similar data. Numerical techniques are then used to develop models relating the experimentally determined dependent (output) data to the independent (input) data. Classical techniques, such as multiple regression, or currently popular techniques, such as neural nets, can be used to develop the relationships.

Knowledge-based models, expert systems, are founded on the use of process knowledge for logically assisting in process control, and fault detection and isolation. Expert systems are based on qualitative and approximate relationships rather than on quantitative relationships. Most papers on expert systems for fault diagnosis suggest that the expert system be used in combination with available quantitative information. That is, the expert system uses all relevant information, including that obtained from a theoretical or empirical process model, for fault diagnosis. This information would include control actions, measurements, and model predictions. Process modeling approaches are not the subject of this report and will not be discussed further except as required for understanding the related material.

\section{GENERATION OF RESIDUALS FOR FAULT DETECTION}

A fault (anomaly, abnormal condition, outlier) occurs when some process variable, e.g., temperature, pressure, or mass of plutonium, is outside of acceptable limits. Such a condition can only be observed by making measurements on a process and comparing them with other, redundant, process information. Frank ${ }^{1}$ places fault detection methods into two general categories defined by the kind of redundant information we possess about the process. These methods are based on either analytical or physical redundancy. Analytical redundancy refers to 
process information obtained from a mathematical or knowledge-based model of the process that is compared with redundant information obtained from measurements. ${ }^{*}$ In contrast, physical redundancy compares the results from redundant measurements. That is, redundant process information is acquired either directly from multiple measurements of the same or related process variables or from duplicate estimates of the same process variables obtained by measurements and model predictions. The data from instruments can be either from sensors or controls. If from controls, it is most generally used as input to a process model of some type.

In the following discussion we will refer to these two general categories of fault detection methods as either model- or measurement-based. An advantage of the model-based approach is that it systematically uses the knowledge that we possess about the process. But if we do not have sufficient knowledge to build a model, the model-based approach may not be practical. Some people consider modeling errors to be the main difficulty in the use of model-based methods for fault detection. ${ }^{2-1}$ An advantage of the measurement-based approach is that it lets the process teach us normal, i.e., controlled, process operation and thus may be applicable to processes that cannot be easily modeled, at least mathematically. Over time a measurementbased approach could provide information needed to develop a model of the process.

\section{A. Model-based Fault Detection}

Model-based fault detection methods require a process model capable of adequately describing the normal operation of the process. Probably the most usual model-based approach involves a comparison of the model-based estimates of state variables with measurements of the same state variables. The state-space representation provides a convenient formalism for representing this system by a pair of equations: the state equation and the measurement equation. The linear, time-varying, discrete forms of these relationships are

State Equation: $\mathbf{x}(t)=\mathbf{A}(t-1) \mathbf{x}(t-1)+\mathbf{B}(t-1) \mathbf{u}(t-1)+\mathbf{L}(t-1) \zeta(t-1)$

Measurement Equation: $\mathbf{y}(t-1)=\mathbf{C}(t-1) \mathbf{x}(t-1)+\mathbf{r}(t-1)$

The vectors $\mathbf{x}, \mathbf{u}, \zeta, \mathbf{y}$, and $\mathbf{r}$ are the $N \times 1, M \times 1, N \times 1, P \times 1$ and $P \times 1$ vectors corresponding to state, input or control, plant noise, measurement or output, and measurement noise respectively. The matrices $\mathbf{A}, \mathbf{B}, \mathbf{C}$, and $\mathbf{L}$ are known and are determined during model development, expressing how the states and controls at time $(t-1)$ relate to the states at time $t$

\footnotetext{
* Traditional materials accounting uses a simple form of analytical redundancy based on a conservation of mass (material balance) model. When the model is correct, the measured masses (inventory, input and output) are used to calculate a residual, the MUF or ID, which is tested to determine if it is significantly different from zero.
} 
and how the states relate to the output measurements. In a chemical system the state variables may be masses, temperatures, pressures, volumes, concentrations, or densities, for example, and they may or may not be directly measurable. In many cases $\mathbf{C}$ is the identity matrix. That is, the measurements are direct, but noisy, observations of the state variables. In some cases, however, the state variables are related to the measured variables through some functionality, e.g., light absorption coefficients in the case of concentrations determined by light absorption spectroscopy. In these cases, $\mathbf{C}$ has a form different from the identity matrix. Temperature and total pressure are examples of state variables that may be directly measured and, in many cases, concentration and solution volume are state variables that are obtained by indirect measurements. For example, in a process tank, the volume is usually determined indirectly from a measurement of liquid level in the tank and a previously determined calibration relating liquid level to tank volume. As written, Eqs. (2-1) and (2-2) are applicable to a system under normal or controlled operating conditions, i.e., no faults.

Two recent review articles $2-1,2-2$ are concerned with model-based fault detection and diagnosis. Several procedures are discussed for redundant variables that must be tested for occurrence of a fault. Three of these procedures are designated as observer, parameter estimation, and parity space methods.

1. Observer Methods. Observer methods involve a comparison of model-based predictions of state variables with measured observations (the observers) of process state variables or variables related to process state variables, such as in Eqs. (2-1) and (2-2). We compare model predictions with measurements using, for example, a Kalman filter approach. The difference or residual, ${ }^{*} \mathbf{r}(t)=\mathbf{y}(t)-\mathbf{C}(t) \mathbf{x}(t)$, from Eq.(2-2) is calculated using the Kalman filter estimate of the state variable, $\mathbf{x}(t)$. If the process is operating normally, i.e., with no faults or anomalies, this residual will be "small" or within an expected range. If, since the most recent measurement, a detectable process fault or sensor failure has occurred, the residual will be "large" enough to fail an appropriate statistical significance test."*

2. Parameter Identification Methods. Parameter identification methods refer to the on-line estimation of mathematical parameters of the process model and their subsequent

\footnotetext{
*This residual difference between the measured and Kalman-filter-based estimate is called the "innovation" in filtering applications.

** If a process fault occurs, the predicted value differs from the measured value because the normal process model does not model faults and thus an incorrect value is predicted. In contrast, if a sensor fails, the predicted value differs from the measured value because of an erroneous measurement. In either case the residual is larger than expected.
} 
comparison with a set of reference parameters developed during normal operations. This approach provides information about the present status of the process, which may not be directly observable or measurable. One parameter identification method models the input-output process behavior by a lumped parameter system of differential equations. Model parameters estimated under current operating conditions are compared with those obtained under normal process operating conditions and a vector of parameter residuals is calculated and tested for faults.

3. Parity Space Approach. Another method, called the parity space approach, uses a selected combination of input and output measurements to make consistency checks based on known relationships between measured variables. Analytical redundancy can involve direct relations between measured variables, e.g., materials balance equations, as well as dynamic difference relationships between inputs and outputs, as in Eqs. (2-1) and (2-2). MUFs are a simple example of a materials balance consistency relation. The materials balance approach is discussed in more detail in a later section.

\section{B . Measurement-based Fault Detection}

Conceptually, measurement-based fault detection methods involve comparing redundant measurements with each other or with target set points and deciding if they signal a fault. Thus, as for model-based fault detection, a significant aspect of measurement-based fault detection entails analyzing residuals and testing for faults.

\section{TESTS FOR OUTLIERS}

After estimating the residuals, it remains to evaluate them by one or more approaches to determine if a fault has occurred. For univariate tests, a number of standard tests are used for this purpose, and we will mention some of them to provide some intuition about the procedures used. Because process faults are outliers in the statistical sense, these tests are identical to statistical methods for detection of outliers. ${ }^{*}$

Before starting, we note that the statistical literature on outliers is vast and it is not our intention to discuss all, or even most, aspects of outliers. Interested readers are invited to peruse this subject for themselves. The text by Barnett and Lewis ${ }^{2-3}$ is a good reference. We

\footnotetext{
*In this work, our primary concern is with multivariate outliers. In the literature, multivariate outlier tests are generally based on extensions of univariate tests. We note that the multivariate data or residuals we expect to acquire from future chemical reprocessing plants will be stochastic because of measurement and other sources of uncertainty, and the variables are likely to be correlated.
} 
will briefly discuss some aspects of outlier detection that are relevant to fault detection in nuclear reprocessing plants from the perspective of nuclear safeguards and NRTA. In this chapter we do not address the issue of statistical methods used for the detection of diversions based on sequences of material unaccounted for (MUFs), which gets into the subject of time series analysis. Chapter III considers this issue.

The fault residual evaluation methods discussed in recent reviews ${ }^{2-1,2-2}$ emphasize identification and isolation of faults as well as fault detection. Fault diagnosis includes identification and isolation, which are more difficult to achieve than is fault detection alone. Fault diagnosis, identification, and isolation require more detailed knowledge of the processes and more numerous and specific measurements. For example, in a three-tank process, a fault, possibly indicative of diversion, could be detected by as few as two measurements: one at the input and one at the output. However, identification of its cause would require additional information, e.g., were the measurements valid? was there some unaccounted holdup in the system? Furthermore, isolation of the fault to a specific place in the process would require additional measurements at locations between the input and output points of the three tanks. Our primary intention here is to discuss fault detection rather than fault identification; however, when a method is applicable to identification, we will mention that fact. The degree of process detail contained in the process model will determine diagnostic capabilities.

\section{A. Outlier Tests Based on the Gaussian Distribution}

The simplest tests are based on the assumption that, under normal no-fault operating conditions, the residuals are solely a result of random noise. The residuals are assumed to follow the Gaussian or normal distribution with mean zero. The variance may be known or estimated from previous data. A fault is indicated if any component of the residual vector lies outside a selected significance value. The test statistic for a univariate residual $r$ that is expected to be zero, $\mathbf{H}_{0}: E(r)=0$ (the alternative hypothesis is $\mathbf{H}_{1}: E(r) \neq 0$ ), is either

$$
z(\alpha)=\frac{r}{\sigma / \sqrt{N}} \text { or } t_{s}(\alpha)=\frac{r}{s / \sqrt{N}}
$$

depending on whether the standard deviation is known or estimated. $N$ is the number of samples used to calculate $r$ and $E$ denotes expected value. The critical values to which these test statistics are to be compared come from the normal or student distributions, respectively. The user specifies what significance level ( $\alpha$ value) will be used to signal a fault. Tests can also be 
based on the $z^{2}$ or $t^{2}$ statistics, which have $\chi_{1}^{2}$ and $F_{1, N-1}$ distributions, respectively. Tests based on the chi-squared distribution are common for testing multivariate residuals.

Multivariate analogs of these simple tests are easily implemented when the assumptions are applicable. For multivariate normal distributions, tests can be used for individual residuals and their $\alpha$ 's adjusted to account for the multiple tests if we wish to maintain the same overall significance level for detecting a fault. The Bonferroni method ${ }^{2-4}$ uses critical values based on $\alpha / p$ where $p$ is the number of individual $z$ or $t$ values being tested. For example, if 10 independent residuals were tested and an overall significance level of 5\% were desired, the individual test values would be compared to a critical value based on a $0.5 \%$ significance level. If the residuals are correlated, this adjustment overcorrects.

Although the assumption of random uncorrelated errors in different measurements may be valid, the multiple residuals calculated from model estimates of different state variables and their corresponding measurements are usually correlated. (See, for example, Eq. (2-8) in Kao, et al.,2-5 or Eq. (2-10) below.) This can be because of materials constraint relations; because some of the state variables are deterministically related, e.g., density and concentration; or because plant noise affects several state variables in a correlated manner. In addition, measurement errors may include a systematic component, for example, from calibration. Thus, for multivariate residuals, one-at-a-time tests based on the assumption of independence are not generally adequate or very powerful, and outlier tests that compensate for the correlations are preferred for multiple-measurement situations. Some of these latter test methods will be discussed later after a discussion on fault detection tests from the literature. ${ }^{2-1,2-2,2-6}$

\section{B . Some Results from the Fault Detection Literature}

The likelihood ratio test ${ }^{2-2}$ is an observer-based method and an extension of the outlier test from Eq. (2-3) that is designed to identify the cause of the fault. This univariate test assumes that the process state variables are perturbed by random white noise, and it requires that the probability distribution functions of the residuals be known for each type of fault, $f_{i}$. This is an observer-based method because the residual vector being tested is the difference between the state estimate of $\mathbf{y}(t)$ and the measured value, i.e., $\mathbf{r}(t)=\mathbf{y}(t)-\mathbf{C}(t) \mathbf{x}(t)$, as mentioned already. Individual elements, $r_{j}$ of $\mathbf{r}(t)$, are used with the alternative hypotheses, $\mathbf{H}_{0}$ :no fault has occurred and $\mathbf{H}_{i}$ : fault $f_{i}$ has occurred. The likelihood ratios, $\left(L_{i j}\right)$, for each $r_{j}$ and each possible fault $f_{i}$ are calculated by Eq. (2-4).

$$
L_{i j}=P\left(r_{j} \mid \mathbf{H}_{i}: f_{i}\right) / \mathrm{P}\left(r_{j} \mid \mathbf{H}_{0}\right) .
$$


The $P$ 's are the probabilities of observing the $r_{j}$ assuming that $f_{i}$ has and has not occurred. The $L_{i j}$ giving the maximum ratio identifies the fault causing the residual. A threshold likelihood value can be assigned for each $f_{i}$, and any $L_{i j}$ exceeding that threshold indicates that the fault may have occurred.

Another test similar to the above requires a different process model for each fault. A residual can then be calculated for each model and used to calculate the likelihoods as in Eq. (2-4). The most likely fault condition is the one giving the largest likelihood. This approach requires a high degree of process knowledge for the development of different models for each type of fault.

The sequential likelihood ratio test 6 is a test that uses the time evolution of the residuals and is popular when time is a factor as will be the usual situation for NRTA safeguards.

$$
L_{i}=\frac{P\left[r_{1}, r_{2}, \ldots, r_{t} \mid \mathbf{H}_{i}\right]}{P\left[r_{1}, r_{2}, \ldots, r_{t} \mid \mathbf{H}_{0}\right]}
$$

Here the $r_{j}$ are scalar residuals corresponding to the measurement at time $t_{j}$ and the $P$ 's are the probabilities of observing the given residuals under fault $f_{i}$ and the no fault condition.

A Bayesian approach for fault detection can be used when probabilities for the occurrences of various kinds of faults are known or can be reasonably approximated.

The materials balance constraint problem has been extensively discussed in the chemical engineering literature where it is called "data reconciliation." As mentioned above, it falls under the "parity space" approach to fault detection. A readable discussion ${ }^{2-7}$ was published in 1985 and the following is based primarily on that article. The problem is one of estimating state variables given a set of measurements of those or related variables when there are linear constraints. With no sensor failures, the measurement model, as in Eq. (2-2), is assumed to be

$$
\mathbf{y}=\mathbf{C x}+\mathbf{e}
$$

where $\mathbf{y}$ is a vector of measured estimates related to the vector of state variables $\mathbf{x}$ through the known matrix $\mathbf{C}$ and $\mathbf{e}$ is a vector of measurement errors assumed to be multivariate normal with mean zero and known covariance matrix $\Sigma$. We will consider a case where $\mathbf{C}$ is the identity matrix,

$$
\mathbf{y}=\mathbf{I x}+\mathbf{e}
$$


The state variables are subject to a set of linear materials balance constraints of the form

$$
\mathbf{G x}=0
$$

We can estimate $\mathbf{x}$ with Eq. (2-7) [and therefore $\mathbf{y}$ with Eq.(2-6)] by constrained weighted least squares

$$
\hat{\mathbf{y}}=\hat{\mathbf{x}}=\left[I-\Sigma \mathbf{G}^{\prime}\left(\mathbf{G} \Sigma \mathbf{G}^{\prime}\right)^{-1} \mathbf{G}\right] \mathbf{y}
$$

And the residual differences between predicted and measured values are

$$
\begin{aligned}
\mathbf{r} & =\mathbf{y}-\hat{\mathbf{y}}=\mathbf{y}-\left[\mathbf{I}-\Sigma \mathbf{G}^{\prime}\left(\mathbf{G} \Sigma \mathbf{G}^{\prime}\right)^{-1} \mathbf{G}\right] \mathbf{y} \\
& =\Sigma \mathbf{G}^{\prime}\left(\mathbf{G} \Sigma \mathbf{G}^{\prime}\right)^{-1} \mathbf{G} \mathbf{y} .
\end{aligned}
$$

Even though the measurement errors e were assumed to be independent or uncorrelated, the residuals $\mathbf{r}$ are not because of $\mathbf{G}$, which constrains the solution. The covariance of $\mathbf{r}$ is

$$
\operatorname{Cov}(\mathbf{r})=\Sigma \mathbf{G}^{\prime}\left(\mathbf{G} \Sigma \mathbf{G}^{\prime}\right)^{-1} \mathbf{G} \Sigma
$$

Tamhane and $\mathrm{Mah}^{2-7}$ described three types of tests that could be used to test outliers under the above model: the global test, the nodal test, and the measurement test.

The global test is a multivariate test based on a chi-squared statistic. According to Eq. (2-7), the expected value of $\mathbf{x}$ is $E[G y]=0$, where the state vector $\mathbf{x}$ has been replaced by its measured approximation $y$ using Eq. (2-6a). Thus, under the null hypothesis of no faults

\footnotetext{
* As an example, consider a single tank with two inflow streams ( $x_{1}$ and $\left.x_{2}\right)$ and two outflow streams ( $x_{3}$ and $x_{4}$ ) with each stream having three chemical constituents and no chemical reactions occuring in the system. We know that at steady state mass balance requires that the inflow and the outflow must sum to zero for each constituent. Thus, we have an equation of the following form for each constituent, $m_{i} ; \Sigma_{j} . m_{i, j} x_{j}=0$ where the sum on $j$ is over the 4 flow streams with outflow stream given negative signs and the $m_{i, j}$ are the mole fractions of constituent $i$ in flow stream $j$. Equation 7 for this system is
}

$$
\left(\begin{array}{llll}
m_{11} & m_{12} & m_{13} & m_{14} \\
m_{21} & m_{22} & m_{23} & m_{24} \\
m_{31} & m_{32} & m_{33} & m_{34}
\end{array}\right)\left(\begin{array}{l}
x_{1} \\
x_{2} \\
x_{3} \\
x_{4}
\end{array}\right)=\left(\begin{array}{l}
0 \\
0 \\
0
\end{array}\right)
$$

The $G$ matrix is composed of the $m_{i j}$, and the flow rates constitute the state vector $\mathbf{x}$. 
$\left(\mathrm{H}_{0}: \mathrm{E}[\mathrm{Gy}]=0\right), \mathrm{Gy}$ has a multivariate normal distribution with a mean of 0 and a covariance matrix of $\mathbf{G} \Sigma \mathbf{G}^{\prime}$. The test statistic

$$
X_{P}^{2}=(\mathbf{G y})^{\prime}\left(\mathbf{G} \Sigma \mathbf{G}^{\prime}\right)^{-1}(\mathbf{G y})
$$

is chi-squared with $P$ degrees of freedom, $P$ being the number of measurements making up $\mathbf{y}$. For example, if we have measurements and constraints for three different chemical components in a process at a specific location, $P=3$. Various techniques can be used with the global test to attempt to identify the fault. For example, in one procedure, the effect on the test statistic of leaving out one measurement is evaluated for each measurement. The measurement found to have the greatest effect on the chi-squared test statistic is said to be the fault location. The Nodal Test could also be implemented.

The Nodal Test is performed on each individual imbalance in $\mathbf{e}=\mathbf{G y}$, as follows:

$$
z_{i}=\frac{\mathrm{e}_{i}}{\operatorname{diag}\left(\mathbf{G \Sigma G ^ { \prime }}\right)^{1 / 2}}
$$

In the absence of faults, each $z_{i}$ is normally distributed with mean 0 and variance of 1 . It is compared with a critical value selected to give an overall $\alpha$ of the desired sensitivity. If $\alpha$ is corrected or adjusted for the $P$ multiple tests, this test is similar to the Bonferroni method mentioned earlier.

The Measurement Test was proposed by Mah and Tamhane ${ }^{2-7}$ because, assuming a correct materials balance model, faults were presumed to result from differences between individual measurements and their predicted values. The individual residuals from Eq. (2-9) are divided by the appropriate variances from Eq. (2-10) as follows.

$$
z_{i}=\frac{r_{i}}{\operatorname{Cov}(\mathbf{r})_{i i}}
$$

where $\operatorname{Cov}(\mathbf{r})_{i i}$ is the diagonal element of $\operatorname{Cov}(\mathbf{r})$ in Eq. (2-10). A fault is present in the $i$ th measurement if $z_{i}$ exceeds the selected critical value. This test is also similar to the Bonferroni test with appropriately adjusted critical values.

These tests for multivariate outliers are either global in that they compare some overall measure of a residual vector or they are specific in that they test individual elements of a residual vector. The various univariate tests differ by the specific error term tested and the assumptions made about the covariance structure. 
C. Some Multivariate Outlier Detection Methods and the Statistical Literature

Beckman and Cook included a brief discussion on multivariate outliers in their general discussion on outliers. ${ }^{2-8}$ For the detection of a single multivariate outlier from multivariate normal samples, the generalized distances from the mean (also referred to as statistical and Mahalanobis distances) are

$$
M d_{i}=\left(\mathbf{y}_{i}-\overline{\mathbf{y}}\right)^{\prime} \Sigma^{-1}\left(\mathbf{y}_{i}-\overline{\mathbf{y}}\right)
$$

if the covariance is known or

$$
M d_{i}=\left(\mathbf{y}_{i}-\overline{\mathbf{y}}\right)^{\prime} \mathbf{S}^{-1}\left(\mathbf{y}_{i}-\overline{\mathbf{y}}\right)
$$

if it is independently estimated. The maximum $M d_{i}$ is compared to critical values from either the chi-squared or weighted $F$ distribution to determine if the corresponding $\mathbf{y}_{i}$ may be an outlier. When several outliers are suspected, computationally intensive iterative approaches can be used to identify the multiple outliers. In these methods a test for $k$ outliers involves comparing the generalized distance when $k$ observations are omitted to the generalized distance when all observations are included.

Stapanian, et al., studied the properties of two popular tests for multivariate outliers, the generalized distance and Mardia's multivariate kurtosis, ${ }^{2-9}$ Eqs. (2-14) and (2-15) respectively. They calculated critical values for both tests for up to 25 dimensions and 500 observations.

$$
m v k=\frac{1}{N} \sum_{i=1}^{N} M d_{i}^{2}
$$

Mardia's multivariate kurtosis is a measure over all the observations and is compared to critical values, which are dependent on $N$ and $P$. Tables for selected values of $N$ and $P$ are provided in Ref. 2-9. The authors conclude that the kurtosis test is better for multiple outliers while the generalized distance metric is superior for single outliers. They caution that, for the kurtosis test, the assumption that all $\mathrm{k}$ outliers, $k>1$, come from the same distribution is probably not valid and thus interpretation of the power of the kurtosis test is limited. They further caution that the kurtosis test was designed for multivariate normal samples and could result in the improper identification of outliers for non-normal distributions.

A major difficulty with the above procedures for multiple outliers is the practical requirement of estimating $\mu$ and $\Sigma$. Because these often must be estimated from the observations, outliers can be masked when sample means and covariance matrices are used. This consideration motivates use of robust estimates for $\mu$ and $\Sigma$. Such robust estimates are designated as 
measures of location and of dispersion to avoid confusion with the sample mean and covariance matrix as conventionally defined. One recently suggested robust estimation method is based on the minimum volume ellipsoid. ${ }^{2-10}$ For location, the center of the minimum volume ellipsoid containing half of the observations is used. For dispersion, the covariance of the same subset of observations is used multiplied by a correction factor found to be necessary for consistency with multivariate normal distributions. Algorithms and equations for these calculations have been published. $2-10$ Using robust estimates for the mean and covariance in Eq. (2-14), a robust distance $\left(r d_{i}\right)$ is calculated that provides a sensitive method for detecting outliers. This procedure requires a fairly large number of observations $N$ compared to variables $P$. Rousseeuw and van Zomeren ${ }^{2-10}$ suggest that this ratio should be greater than five, a value consistent with other empirical results in multivariate analysis.

\section{Outliers Using Principal Components Analysis. Gnanadesikan $2-11$ distin-} guishes two categories of statistical analysis of multivariate problems as 1) analysis of internal structure, e.g., principal components analysis, cluster analysis, and multidimensional scaling and 2) analysis of external structure, such as multiple regression and multivariate analysis of variance. Each of these categories gives multivariate residuals. Principal components analysis* is often used as a dimensionality reduction method when correlated variables are present. For data vectors containing $P$ elements, it may be assumed that the components corresponding to the $Q(Q \leq P)$ largest eigenvalues explain the important internal structure of the data. The lack of fit lof after implementation of $Q$ principal components, for an individual observation $i$ is

$$
(\text { lof })_{i}^{2}=\sum_{j=Q+1}^{P} t_{i, j}^{2},
$$

where $t_{i, j}$ is the score for observation $i$ on principal component $j$. If we assumed that $Q$ principal components sufficiently modeled or reconstructed the data matrix $\mathbf{Y}$ then large values of $d_{i}^{2}$ are indicative of outlier observations.

\footnotetext{
${ }^{*}$ Consistent with our notation, we denote a set of $N$ objects or individuals for which we have observed values of $P$ variables by the $N \times P$ matrix $Y$. For convenience we assume the columns of $Y$ have been mean centered. A complete principal component analysis of $\mathrm{Y}$ is the matrix decomposition $\mathrm{Y}=\mathrm{TV}^{\prime}$ where $\mathrm{V}$ is a $P \times Q$ matrix whose columns are the orthonormal eigenvectors of $Y^{\prime} Y$ with eigenvalues $\lambda_{q}$, and $T$ is the $N \times Q$ matrix of principal component scores, and $\mathbf{T}^{\top} \mathbf{T}=\operatorname{diag}\left(\lambda_{q}\right)$ is the covariance of $\mathrm{T}$. Note that $Q$ is less than or equal to $P$ depending on the rank of $Y$. Because of the orthonormal property of $V, T=Y V$. Computationally the complete principal components analysis can be conveniently calculated using the singular value decomposition (SVD) of either $\mathbf{Y}$ or $\mathbf{Y}^{\prime} \mathbf{Y}$. For the latter, $\operatorname{SVD}\left(\mathbf{Y}^{\prime} \mathbf{Y}\right)=\mathbf{V} \operatorname{diag}\left(\lambda_{q}\right) \mathbf{V}^{\prime}$. Thus in the reconstruction of $\mathbf{Y}$ from the eigenvectors, first the scores corresponding to the desired principal components are calculated using $\mathbf{T}^{*}=\mathbf{Y}$ $V^{*}$, where the * denotes that only the desired eigenvectors are included in $\mathbf{V}$. Second the reconstructed data, $\mathbf{Y}$, are calculated using these scores by $\mathbf{Y}=\mathbf{T}\left(\mathbf{V}^{*}\right)^{\prime}$.
} 
Another approach, 2-12 analogous to the leverage concept from multiple linear regression, that provides a measure of the influence of an observation on the principal components analysis is defined by

$$
h_{i}-\frac{1}{N}=\sum_{j=1}^{P} \frac{t_{i, j}^{2}}{\lambda_{j}},
$$

where $\lambda_{j}$ is the $j$ th eigenvalue. This is equivalent to summing the square of $z$-scores corresponding to each principal component for each observation. The $h_{i}$ can be expressed as a sum of two terms corresponding to the components considered to be "important" to the internal structure and those components assumed not important. ${ }^{2-12}$ Components not important to the internal data structure are those presumed to be modeling only random noise. Thus

$$
h_{i}-\frac{1}{N}=\sum_{j=1}^{Q} \frac{t_{i, j}^{2}}{\lambda_{j}}+\sum_{j=Q}^{P} \frac{t_{i, j}^{2}}{\lambda_{j}}=h_{1 i}+h_{2 i}
$$

The $h_{1 i}$ and $h_{2 i}$ are generalized or Mahalanobis distances from the means of the projections of $\mathbf{Y}$ into the two orthogonal spaces modeled by the corresponding sets of principal components. Thus a large $h_{1 i}$ indicates an observation that is an outlier compared to the systematic structure of $\mathbf{Y}$, and a large $h_{2 i}$ indicates a sample that is an outlier with respect to the noise or random structure in $\mathbf{Y}$.

Another influence measure discussed by Naes $2-12$ is

$$
(\text { Inf })_{i, j}=-\mathbf{t}_{i, j} N \sum_{\substack{k=1 \\ k \neq j}}^{P} \frac{\mathbf{V}_{k} \mathbf{t}_{i, k}}{\lambda_{k}-\lambda_{j}}
$$

(Inf $)_{i, j}$ is a $P$ element vector, giving the influence of observation $i$ on each of the $P$ elements of the $j$ th eigenvector. $V_{k}$ is the $k$ th eigenvector of $Y^{\prime} \mathbf{Y}$. According to Naes ${ }^{2-12}$ there is a problem in using this measure because it is not scaled and no test statistic is available. He used visual inspection of the norms of $(\mathbf{I n f})_{i, j}$ together with the leverage measures to identify outliers.

Another approach used with principal components analysis is presented in a paper on multivariate process control by Jackson. $2-13$ Jackson suggests rescaling the eigenvectors by the eigenvalues so that each eigenvector has unit variance.

$$
\mathbf{W}=\mathbf{V} \operatorname{diag}(\lambda)^{-1 / 2}
$$


This has the effect of putting the scores for each principal component on the same scale so that each set of scores now has a mean of zero and a variance of one. This modified score matrix, similar to the above $\mathbf{T}$ matrix, is calculated according to Eq. (2-21).

$$
\mathbf{T}^{\mathbf{z}}=\mathbf{Y} \mathbf{W}^{\prime}
$$

where we have used the superscript $\mathbf{z}$ to denote that the scores have been scaled. Now the $t_{i, j}^{z}$ are the scores for observation $i$ on scaled principal component $j$ and can be tested directly against the $\mathrm{z}$ scores from the normal distribution to determine if the particular principal component score may be an outlier. In addition, the Mahalanobis distances are easily calculated directly from the scores. For observation $i$ this is,

$$
M d_{i}=\mathbf{t}_{i}^{\mathrm{z}}\left(\mathbf{t}_{i}^{\mathrm{z}}\right)^{\prime}
$$

Note that this approach is essentially the same as that represented by Eq. (2-17). Equation (2-22) provides a convenient metric for testing the scores after each additional principal component has been added to the model to determine what observations were "fit" by the additional component.

Given a set of observations, one approach would be to develop a principal components model using only the $Q$ principal components needed to fit "good" observations, i.e., those with no faults and only random noise. Eigenvectors corresponding to "small" eigenvalues would be assumed to contain only random noise. Future observations, $y$, could be tested based on how well they are fit by the principal components model developed for no fault models. Jackson suggests an evaluation procedure for future observations that considers the total residual not explained by the principal components model $(\text { lof })_{i}^{2}$ from Eq. (2-16), $M d_{i}$ from Eq. (2$22)$, and the individual $t_{i j}$ from Eq. (2-21) in sequence. Note that $y$ is the observed data vector appropriately mean centered and scaled and $\hat{\mathbf{y}}$ is its estimate from the principal components model composed of $Q$ principal components.

In addition to these approaches, other techniques can be used during the model development phases of this procedure that take advantage of numeric as well as graphical interpretation of the data to ensure that the models developed do not inadvertently include outliers. For example, visualization by use of two- or three-dimensional plots of the principal component scores can be useful in determining data structure and in identifying outliers.

2. Literature Example from a Reprocessing Plant. In a recent paper ${ }^{2-14}$ analytical data from spent nuclear fuel processing was interpreted by principal components analysis. 
A total of 16 variables, including a measure of burnup, plutonium and uranium isotope concentrations, and isotopic ratios, were reported for 10 different reprocessing batches. These data (Table I in Ref. 2-14) were used as the $Y$ matrix for a principal components analysis. Based on eigenvector and score plots and eigenvalues, the author concluded that all of the samples belonged to the set, i.e., there were no outliers. We have applied some of the above tests to these same data. Because the scales of the distinct variables differed by several orders of magnitude, each variable was mean centered and normalized by its standard deviation prior to principal components analysis. For these data 3 principal components explained $92 \%$ and 4 principal components explained $97 \%$ of the (normalized) variance. Thus, only a 3- or 4component model would be needed to adequately describe these observations.

The Mahalanobis distances, $M d_{i}$ in Eq. (2-22), were calculated as additional principal components were added to the principal component model and are listed in Table 2-I. The critical values, $\alpha=0.05$, based on the scaled $F$ statistic are in the last row. The ratios of these distances to the critical values are plotted in Fig. 2-1 for the first three principal components. Because none of these ratios are close to 1.0 , there are no outliers, regardless of the number of principal components used to model the data. This conclusion is consistent with that reported in Ref. 2-14. The normalized $t_{i, j}$ scores from Eq. (2-21) are plotted in Fig. 2-2 as PC 2 or PC 3 versus PC 1. Recall that these are in the form of $z$ scores and thus can be evaluated as the number of standard deviations from the mean. Only one of the scores (Sample 1 with PC 2) is larger than two sigma, $t_{1,2}=2.24$. This is to be expected from the $M d_{i}$ values. Normally one would not even look at the $t_{i, j}$ scores with these $M d_{i}$ values. Future observations could be subjected to the same model to test for outliers.

Table 2-I. Mahalanobis Distances for the Reprocessing Data as Principal Components are Added

\begin{tabular}{clllll}
\hline & \multicolumn{5}{c}{ Number of Principal components $P$} \\
\cline { 2 - 6 } Observation & 1 & \multicolumn{5}{c}{3} & \multicolumn{1}{c}{ C } & \multicolumn{1}{c}{5} \\
\hline 1 & 0.36 & 5.38 & 5.61 & 5.99 & 6.38 \\
2 & 2.08 & 2.33 & 2.92 & 2.94 & 4.69 \\
3 & 0.05 & 0.09 & 1.82 & 8.03 & 8.05 \\
4 & 0.24 & 0.41 & 1.57 & 1.59 & 1.73 \\
5 & 1.17 & 1.60 & 1.60 & 1.66 & 1.73 \\
6 & 0.06 & 0.07 & 0.76 & 1.32 & 1.45 \\
7 & 1.91 & 3.04 & 3.18 & 3.19 & 3.28 \\
8 & 0.13 & 1.63 & 3.21 & 4.07 & 5.94 \\
9 & 2.81 & 3.26 & 3.26 & 3.82 & 4.97 \\
10 & 0.20 & 0.20 & 3.06 & 3.39 & 6.77 \\
Critical Value, & 4.96 & 9.11 & 13.91 & 19.89 & 27.75 \\
$\alpha=5 \%$ & & & & & \\
\hline
\end{tabular}




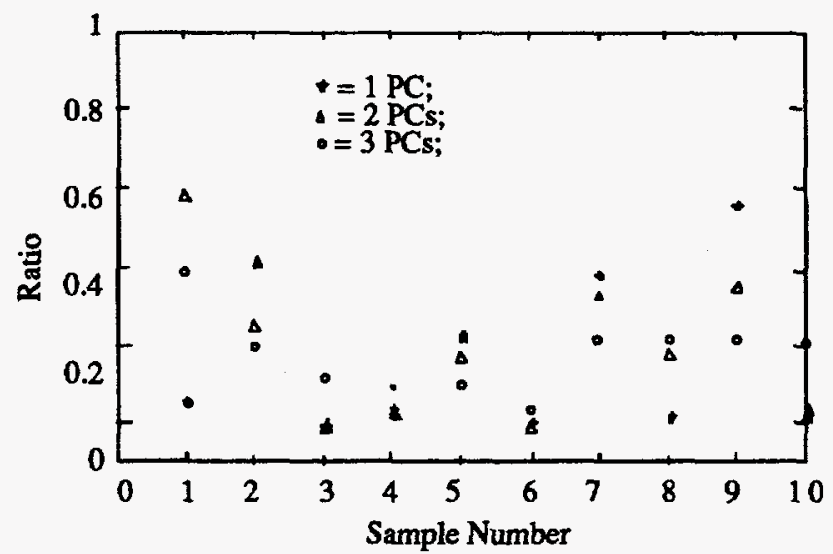

Fig. 2-1. Ratio of Mahalanobis distance to $5 \%$ critical values for either 1,2 , or 3 principal components (PCs) in the model.
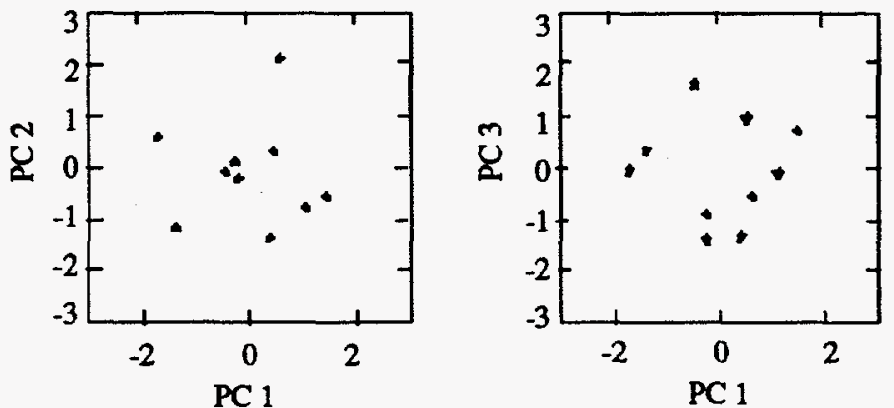

Fig. 2-2. Scores of PC 2 and PC 3 plotted against that of PC 1 for the 10 samples.

\section{THREE-TANK PROBLEM}

In this section we demonstrate near-real-time fault detection for a simulated chemical system in which several variables are measured. The system consists of three connected tanks containing three non-reacting chemical species. This system is sufficiently complex yet simple enough to be accurately modeled using a fundamental approach. For example, in this treatment, temperature is assumed to be constant. More realistically, if the solution transfer is by air lift or pump, then temperature will change.

\section{A. The Three-Tank Model}

The three-tank system is diagrammed in Fig. 2-3. Observe that solution flows from left to right and that each tank has in-flows and out-flows. We assume single-phase aqueous solutions and perfectly mixed tanks. The dynamics are described by a system of coupled differential equations based on total mass balances for each tank and on individual mass balances for each chemical species. These mass balances are expressed by the following word equation.

$$
\text { [Time rate of change of mass }]=[\text { Mass in }]-[\text { Mass out }] \text {. }
$$




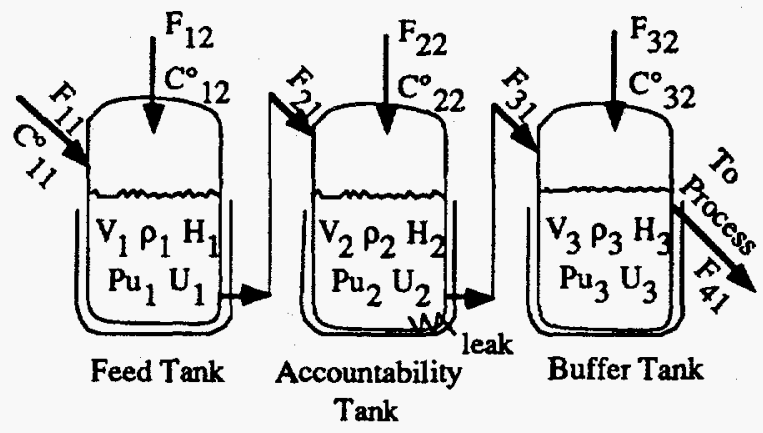

Fig. 2-3. A three-tank system with three non-reacting constituents.

For total mass, if density is constant or a linear function of concentrations, the total mass balance for Tank 1 is

$$
d V_{1} / d t=F_{11}+F_{12}-F_{21}
$$

All symbols are as indicated in Fig. 2-3. For each chemical constituent in Tank 1, we have a relation like Eq. (2-25) for the rate of change of constituent mass in the tank.

$$
d\left(C_{1} V_{1}\right) / d t=C_{1} d V_{1} / d t+V_{1} d C_{1} / d t=C_{11}^{0} F_{11}+C_{12}^{0} F_{12}-F_{21} C_{1}
$$

Where $C$ represents concentration of either nitric acid, plutonium, or uranium. Substituting for $d V_{1} / d t$ from Eq. (2-24), rearranging and eliminating common terms gives

$$
\frac{d C_{1}}{d t}=\frac{\left[\left(C_{11}^{0} F_{11}+C_{12}^{0} F_{12}\right)-\left(F_{11}+F_{12}\right) C_{1}\right]}{V_{1}}
$$

It may appear surprising that, following the substitution, the flow out of the tank, $F_{21}$, from Eq. (2-24) does not appear in Eq. (2-26); however, its effect is realized through $V_{1}$ and Eq. (2-24). Equations similar to (2-24) and (2-26) are written for each tank. The entire system of coupled differential equations for the three tanks is given in Table 2-II.

For constant flows the volume equations can be easily integrated; however, the concentration equations are more complicated. In a process plant the $F_{i j}$ would be measured input or control variables, and thus, for real time modeling as in process control, their measured values would vary with time. These variations would be caused by both fluctuations in the true flow rates and measurement errors. For these reasons we treat the flows as stochastic inputs to the model. To maximize modeling flexibility, the system of differential equations is solved by numerical methods. 
Table 2-II. Differential Equations for the Three-Tank, Three-Specie Problem ${ }^{a, b}$

$$
\begin{aligned}
& d V_{1} / d t=F_{11}+F_{12}-F_{21} \\
& d H_{1} / d t=\left[\left(H_{11}^{0} F_{11}+H_{12}^{0} F_{12}\right)-\left(F_{11} F_{12}\right) H_{1}\right] / V_{1}
\end{aligned}
$$

Tank $1=$

$$
\begin{aligned}
& d P u_{1} / d t=\left[\left(P u_{11}^{0} F_{11}+P u_{12}^{0} F_{12}\right)-\left(F_{11} F_{12}\right) P u_{1}\right] / V_{1} \\
& d U_{1} / d t=\left[\left(U_{11}^{0} F_{11}+U_{12}^{0} F_{12}\right)-\left(F_{11} F_{12}\right) U_{1}\right] / V_{1}
\end{aligned}
$$

Tank $2=$

$$
\begin{aligned}
& d V_{2} / d t=F_{21}+F_{22}-F_{31} \\
& d H_{2} / d t=\left[\left(H_{1} F_{21}+H_{22}^{0} F_{22}\right)-\left(F_{21} F_{22}\right) H_{2}\right] / V_{2}
\end{aligned}
$$

$$
\begin{aligned}
& d P u_{2} / d t=\left[\left(P u_{1} F_{21}+P u_{22}^{0} F_{22}\right)-\left(F_{21} F_{22}\right) P u 2\right] / V_{2} \\
& d U_{2} / d t=\left[\left(U_{1} F_{21}+U_{22}^{0} F_{22}\right)-\left(F_{21} F_{22}\right) U_{2}\right] / V_{2}
\end{aligned}
$$

Tank $3=$

$$
\begin{aligned}
& d V_{3} / d t=F_{31}+F_{32}-F_{41} \\
& d H_{3} / d t=\left[\left(H_{2} F_{31}+H_{32}^{0} F_{32}\right)-\left(F_{31} F_{32}\right) H_{3}\right] / V_{3}
\end{aligned}
$$

$$
\begin{aligned}
& d P u_{3} / d t=\left[\left(P u_{3} F_{31}+P u_{32}^{0} F_{32}\right)-\left(F_{31} F_{32}\right) P u 3\right] / V_{3} \\
& d U_{3} / d t=\left[\left(U_{3} F_{31}+U_{32}^{0} F_{32}\right)-\left(F_{31} F_{32}\right) U_{3}\right] / V_{3}
\end{aligned}
$$

a $V, H, P u$, and $U$ denote volumes and concentrations of nitric acid, plutonium, and uranium respectively.

bSolution densities are modeled by an empirical equation relating density to concentrations of nitric acid, plutonium, and uranium developed by Watson and Rainey. Expressed in terms of molar concentrations at $25^{\circ} \mathrm{C}$, this is $\rho=$ $0.99707+0.032201 \mathrm{H}+0.36240 \mathrm{Pu}+0.32185 \mathrm{U}$.

So that stochastic flow rates in a real time scenario can be modeled, we used the Euler method with constant time steps that are short enough to provide the desired accuracy. The user must specify initial concentrations and volumes in the tanks as well as the flow rates during the simulation. The variables that must be specified are listed in Table 2-III. 
Table 2-III. Variables that Must be Specified by the User in the Three-Tank Simulation

Initial volumes and concentrations

of $\mathrm{HNO}_{3}$, plutonium, and uranium

in each tank

$$
\begin{aligned}
& V_{1}^{0}, V_{2}^{0}, V_{3}^{0} \\
& H_{1}^{0}, P u_{1}^{0}, U_{1}^{0} \\
& H_{2}^{0}, P u_{2}^{0}, U_{2}^{0} \\
& H_{3}^{0}, P u_{3}^{0}, U_{3}^{0}
\end{aligned}
$$

Concentrations of $\mathrm{HNO}_{3}$, plutonium, and uranium in the external input flows: $F_{11}, F_{12}, F_{22}$, and $F_{32}$

Flow rates during the simulation Start and stop times
$\mathrm{C}_{i j}^{0}=\mathrm{HNO}_{3}$, plutonium, and uranium for tank $i$ and input flow $j$
$F_{11}, F_{12}, F_{21}, F_{22}, F_{31}, F_{32}, F_{41}$

$t_{0}, t_{1}$

For given input flows, initial tank volumes, and initial concentrations of $\mathrm{HNO}_{3}$, plutonium, and uranium, the differential equations are solved to give the outputs, i.e., the volumes and concentrations in the tanks at various times. These volumes and concentrations are the model predictions. The model predictions are compared to "measured" values for purposes of fault detection. For these simulations the "measured" values are obtained from model predictions by the application of measurement uncertainty. Note that model predictions also depend on measurements, i.e., the initial conditions and the flow rates, meaning that both predictions and measurements are subject to uncertainty that must be accounted for when testing the significance of model/measurement differences.

\section{B . Model Predictions and Uncertainty Under Normal (No Fault) Operations}

One thousand simulations were performed to provide a data set for normal operating conditions and from which statistical parameters could be estimated. The flow rates and initial tank conditions were the same for each simulation except for the application of randomly distributed uncertainty to measured variables. For the initial tank conditions, the uncertainties were applied once at the beginning of each simulation. For the flow rates we assumed that new measured values became available every $0.1 \mathrm{~h}$ at which time the model values were updated." The measurement errors used are listed in Table 2-IV.

\footnotetext{
*Notice that with this approach the use of time-varying inputs is straightforward.
} 


\section{Table 2-IV. One-Sigma Percent Relative Standard Deviations Assumed for Flow Rates and Initial Volumes and Concentrations}

\begin{tabular}{cccccc}
\hline Flow rates & Volume & Nitric acid & Plutonium & Uranium & Density \\
\hline 5.0 & 0.2 & 1.0 & 0.2 & 0.4 & 0.2 \\
\hline
\end{tabular}

Results of these simulations are given in Table 2-V, where the true values at the end of a 10.0-h simulation were based on a model run assuming no measurement uncertainty in initial conditions and flow rates. The 1000 simulations were obtained using an Euler method with flow uncertainties applied every $0.1 \mathrm{~h}$.

\begin{tabular}{|c|c|c|c|c|c|}
\hline \multirow[b]{2}{*}{ Variable } & \multirow[b]{2}{*}{ Tank } & \multicolumn{3}{|c|}{1000 Simulations } & \multirow[b]{2}{*}{$\begin{array}{l}\text { Percent Relative } \\
\text { Standard Deviation }\end{array}$} \\
\hline & & $\begin{array}{l}\text { True } \\
\text { Value }\end{array}$ & $\begin{array}{l}\text { Mean } \\
\text { Value }\end{array}$ & $\begin{array}{c}\text { Standard } \\
\text { Deviation }\end{array}$ & \\
\hline Volume & $\begin{array}{l}1 \\
2 \\
3\end{array}$ & $\begin{array}{l}100.00 \\
152.50 \\
105.01\end{array}$ & $\begin{array}{r}99.98 \\
152.49 \\
105.01\end{array}$ & $\begin{array}{l}0.7210 \\
0.622 \\
0.374\end{array}$ & $\begin{array}{l}0.72 \\
0.41 \\
0.36\end{array}$ \\
\hline Nitric Acid & $\begin{array}{l}1 \\
2 \\
3\end{array}$ & $\begin{array}{l}5.000 \\
5.000 \\
5.000\end{array}$ & $\begin{array}{l}4.979 \\
5.000 \\
4.993\end{array}$ & $\begin{array}{l}0.117 \\
0.049 \\
0.126\end{array}$ & $\begin{array}{l}2.35 \\
0.98 \\
2.52\end{array}$ \\
\hline Plutonium & $\begin{array}{l}1 \\
2 \\
3\end{array}$ & $\begin{array}{l}0.8160 \\
0.8160 \\
0.4080\end{array}$ & $\begin{array}{l}0.8157 \\
0.8159 \\
0.4080\end{array}$ & $\begin{array}{l}0.0054 \\
0.0021 \\
0.0017\end{array}$ & $\begin{array}{l}0.66 \\
0.26 \\
0.41\end{array}$ \\
\hline Uranium & $\begin{array}{l}1 \\
2 \\
3\end{array}$ & $\begin{array}{l}1.920 \times 10^{-2} \\
1.920 \times 10^{-2} \\
0.960 \times 10^{-2}\end{array}$ & $\begin{array}{l}1.919 \times 10^{-2} \\
1.920 \times 10^{-2} \\
0.960 \times 10^{-2}\end{array}$ & $\begin{array}{l}0.0246 \times 10^{-2} \\
0.0096 \times 10^{-2} \\
0.0048 \times 10^{-2}\end{array}$ & $\begin{array}{l}1.28 \\
0.50 \\
0.50\end{array}$ \\
\hline Density & $\begin{array}{l}1 \\
2 \\
3\end{array}$ & $\begin{array}{l}1.4600 \\
1.4600 \\
1.3090\end{array}$ & $\begin{array}{l}1.4598 \\
1.4599 \\
1.3088\end{array}$ & $\begin{array}{l}0.0042 \\
0.0017 \\
0.0041\end{array}$ & $\begin{array}{l}0.29 \\
0.12 \\
0.31\end{array}$ \\
\hline
\end{tabular}

The data in Table 2-V demonstrate the uncertainties in model predicted volumes, concentrations, and densities at the end of the simulation when 5\% relative errors in flow rates and smaller relative errors (Table 2-IV) in initial volumes and concentrations are assumed. 
Measured Values: Measured values $(1000 \times 15)$ were calculated for each of the $(1000 \times 15)$ model predictions by application of the random uncertainties listed in Table 2-IV to the true values in Table 2-V using a Gaussian random number generator.

Residuals: The differences between the modeled and measured values define a $1000 \times$ 15 matrix of residuals that represent a set of no-fault operations. Future residuals will be compared to these to test for the presence of potential faults; mean values and the covariance structure are estimated from these. Because $N=1000$, the sample mean residual vector $\overline{\mathbf{r}}$ and covariance matrix $S_{r}$ are taken to be the true $\mu$ and $\Sigma$.

Analysis of Normal (no-fault) Residuals: The residuals representing normal operations were evaluated by calculation of the Mahalanobis distances and by principal components analysis. The $M d s$, which should be approximately chi-squared with 15 degrees of freedom, are plotted in Fig. 2-4. The $1.0 \%$ and $0.1 \%$ probabilities from $\chi_{15}^{2}$ are also indicated. Of the 1000 residuals, 10 lie between these two probabilities indicating that the data are distributed as expected.

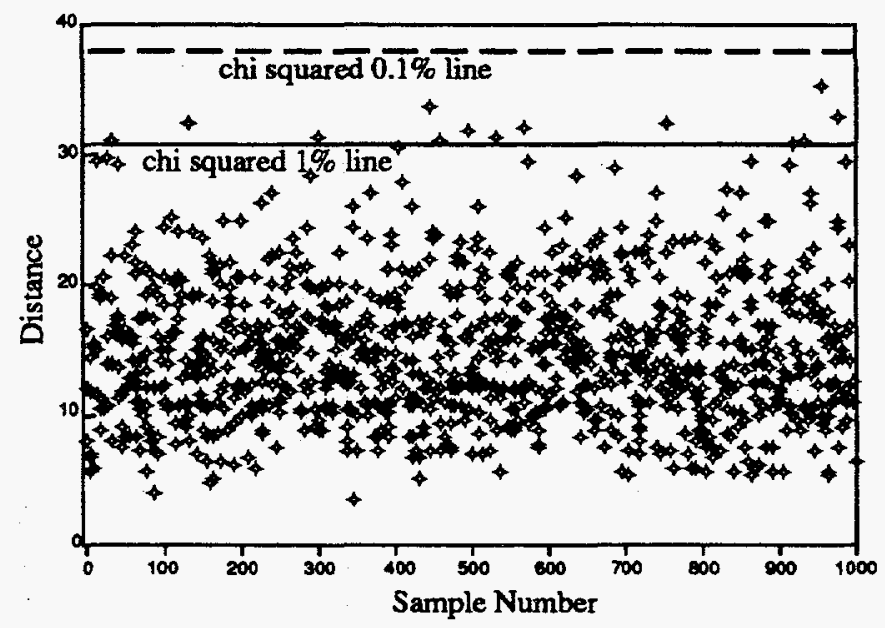

Fig 2-4. Mahalanobis distances of the 1000 residuals constituting the normal (no fault) data.

Principal components analysis of these residuals was not useful in reducing dimensionality. Twelve components were required to reproduce $95 \%$ of the variance for the residuals and, more importantly, because the 15th principal component accounted for a significant portion of the variance of plutonium and density in Tank 1 , it must be retained. 


\section{Diversion Scenarios}

Two diversion scenarios were investigated: 1) a steady leak from Tank 2 without replacement and 2) the same leak but the lost solution is replaced with water. We used the model to simulate leaks to enable accurate estimation of the volumes, concentrations, and densities in the tanks after the 10-h simulations. The measured values were obtained by application of measurement uncertainty to these model results. In practice, the model would not know about the leak and thus would make erroneous predictions because it assumes normal operations. The true conditions, i.e., the loss because of leakage, are represented by the measured data. The residuals are the differences between measured and modeled data, which fault detection methods are designed to detect. ${ }^{*}$ The following discussion assumes measurements were made and compared with predictions at the end of the 10-h time interval.

1. Leak without Replacement. The first diversion scenario is simulated as a steady leak from Tank 2 without replacing the lost volume. During ten hours of operation, continuous leaks of $0.1,0.2,0.25,0.3,0.5$, and $1.0 \mathrm{~L} / \mathrm{h}$, for total volume losses of $1,2,3,5$, and $10 \mathrm{~L}$, were simulated in separate runs. These correspond to total diversions of $195,390,488,585$, 975 , and $1950 \mathrm{~g}$ of plutonium from Tank 2 over a period of ten hours. Because the final volume in Tank 2 is about $152 \mathrm{~L}$ (Table 2-V), these diversions amount to total volume shortages of from $0.66 \%$ to $6.6 \%$ at the end of the simulation.

The $0.5-\mathrm{L} / \mathrm{h}$ leak is easily detected and will be used for discussion purposes and for comparison with the same leak with replacement. Numerical results are in Table 2-VI. The Tank 2 volume residual (measured minus modeled) of -4.69 (vs a theoretical value of -5.01 ) was clearly detectable as evidenced by a $z$-statistic of -6.7 . The loss of this volume has virtually no effect on concentrations as evidenced by the insignificant $z$-scores for all of the remaining variables. The effect of this volume loss on SNM mass would be reflected in the product of volume and concentration, which gives losses of $848 \mathrm{~g}$ and $21.5 \mathrm{~g}$ for plutonium and uranium, respectively. The $z$-scores for all the variables are plotted in Fig. 2-5 together with the 5\% probability levels.

Mahalanobis distances can be calculated using the original data or the data following principal components analysis. If all principal components are used in the calculation, the distances will be identical. In Fig. 2-6, $M d s$ are plotted as additional principal components are added to the calculation. The plot indicates a distance of 72 when all 15 principal components

\footnotetext{
*It should be noted that, when residuals are calculated only at the end of a time interval, the effect of a small steady leak is similar to that of a single large leak of the same total volume. However, if measurements are made more frequently and residuals calculated for each measurement, then the effects may differ more substantially.
} 


\begin{tabular}{|c|c|c|c|c|c|}
\hline Tabl & $\begin{array}{ll}\text { VI. Leak } \\
\text { Result }\end{array}$ & $\begin{array}{l}0.5 \mathrm{~L} / \mathrm{h} \text { wit } \\
\text { n Individu }\end{array}$ & $\begin{array}{l}\text { Replace } \\
\text { ariablesa }\end{array}$ & It - Su & $y$ of \\
\hline Variable & $\begin{array}{l}\text { Predicted } \\
\text { Value }\end{array}$ & $\begin{array}{l}\text { Measured } \\
\text { Value }\end{array}$ & Residual & z-score & $s_{r}$ \\
\hline Vol $_{1}$ & 100.4437 & 100.7744 & 0.3308 & 0.4425 & 0.7475 \\
\hline $\mathrm{Vol}_{2}$ & 152.0864 & 147.3954 & -4.6910 & -6.6956 & 0.7006 \\
\hline $\mathrm{Vol}_{3}$ & 104.5693 & 104.4166 & -0.1528 & -0.3601 & 0.4242 \\
\hline $\mathrm{HNO}_{3}$ & 5.1357 & 5.1127 & -0.0230 & -0.1840 & 0.1250 \\
\hline $\mathrm{HNO}_{3}$ & 5.0390 & 5.0045 & -0.0345 & -0.5014 & 0.0688 \\
\hline $\mathrm{HNO}_{3}$ & 4.8312 & 4.8300 & $-1.16 e-3$ & -0.0086 & 0.1342 \\
\hline $\mathrm{Pu}_{1}$ & 0.8221 & 0.8209 & $-1.15 \mathrm{e}-3$ & -0.2035 & $5.65 e-3$ \\
\hline $\mathrm{Pu}_{2}$ & 0.8192 & 0.8212 & $1.97 e-3$ & 0.7314 & $2.67 e-3$ \\
\hline $\mathrm{Pu}_{3}$ & 0.4100 & 0.4103 & $3.12 \mathrm{e}-4$ & 0.1660 & $1.88 \mathrm{e}-3$ \\
\hline $\mathrm{U}_{1}$ & 0.0196 & 0.0195 & $-9.64 e-5$ & -0.3804 & $2.53 e-4$ \\
\hline $\mathrm{U}_{2}$ & 0.0193 & 0.0193 & $-1.53 e-5$ & -0.1239 & $1.23 e-4$ \\
\hline $\mathrm{U}_{3}$ & 0.0096 & 0.0096 & $1.85 e-5$ & 0.3078 & $6.02 e-5$ \\
\hline$\rho_{\underline{1}}$ & 1.4667 & 1.4664 & $-2.73 e-4$ & -0.0519 & $5.26 e-3$ \\
\hline$\rho_{2}$ & 1.4624 & 1.4627 & $3.27 \mathrm{e}-4$ & 0.0957 & $3.42 \mathrm{e}-3$ \\
\hline$\rho_{3}$ & 1.3043 & 1.3034 & $-9.24 e-4$ & -0.1896 & $4.87 e-3$ \\
\hline
\end{tabular}
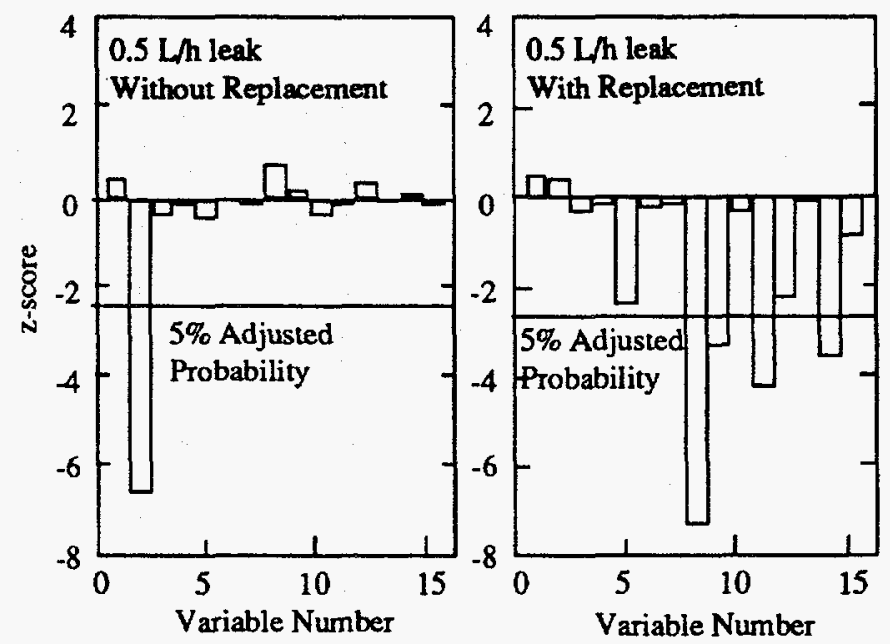

Fig. 2-5. The $z$-scores for residuals corresponding to each of the 15 variables for the $0.5-\mathrm{L} / \mathrm{h}$ leak with and without replacement of the volume removed. 

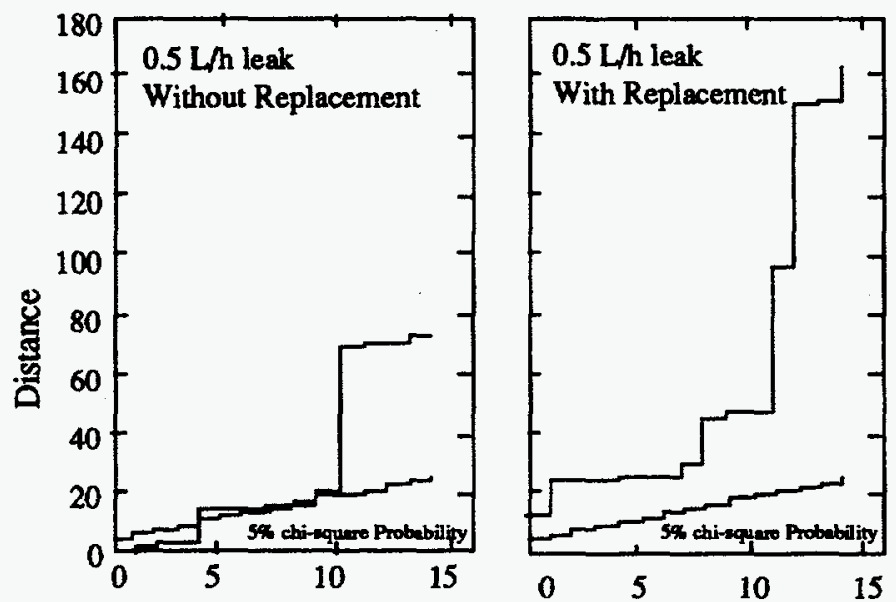

Fig. 2-6. Mahalanobis distances as number of principal components increases from 1 to 15. The distance for 15 principal components is the same as that using the original data.

are used. As exhibited in the figure, the $5 \%$ chi-squared probability for 15 variables is 25 , thus the value of 72 for the $0.5 \mathrm{~L} / \mathrm{h}$ leak is clearly detected as a fault. It is instructive to observe the shape of the curve in Fig. 2-6. Large increases in $M d$ occur when principal components 5 and 11 are added, with the increase for 11 being particularly notable. This suggests that specific principal components may provide more sensitive tests for certain types of diversions than any single original variable.

A univariate probability level of $5 \%$ is indicated in Fig. 2-7 where individual principal component scores are plotted. Because of the way the principal components were normalized, the principal component scores are z-scores and thus directly comparable to areas of the normal probability distribution. The probability value plotted has been adjusted for multiple tests. That is, the desired probability value is divided by the number of tests to obtain an adjusted $z$-score. For 15 variables and a probability of $5 \%$ this gives an adjusted probability of $0.33 \%$ with a corresponding $z$-score of 2.72. Figure 2-7 illustrates that principal components 5 and 11 are detected as faults or outliers using this criterion. As for $M d \mathrm{~s}$, the importance of principal component number 11 is demonstrated by this plot.

Results of the tests for all leaks under the no replacement scenario are summarized in Table 2-VIIa. Using these procedures a leak has to be about $0.25 \mathrm{~L} / \mathrm{h}$ or larger to be detected at the 5\% significance level. Univariate tests for principal component 11 and the volume in Tank 2 are about equally sensitive at detecting a diversion under these conditions. Using 11 principal components, the Mahalanobis distances in Table 2-VIIb detect a diversion at $0.3 \mathrm{~L} / \mathrm{h}$ but not at $0.25 \mathrm{~L} / \mathrm{h}$. The Mahalanobis distance based on all variables does not detect the $0.3 \mathrm{~L} / \mathrm{h}$ leak at the $5 \%$ level indicating a small advantage for the principal component approach when using the Mahalanobis distance. 

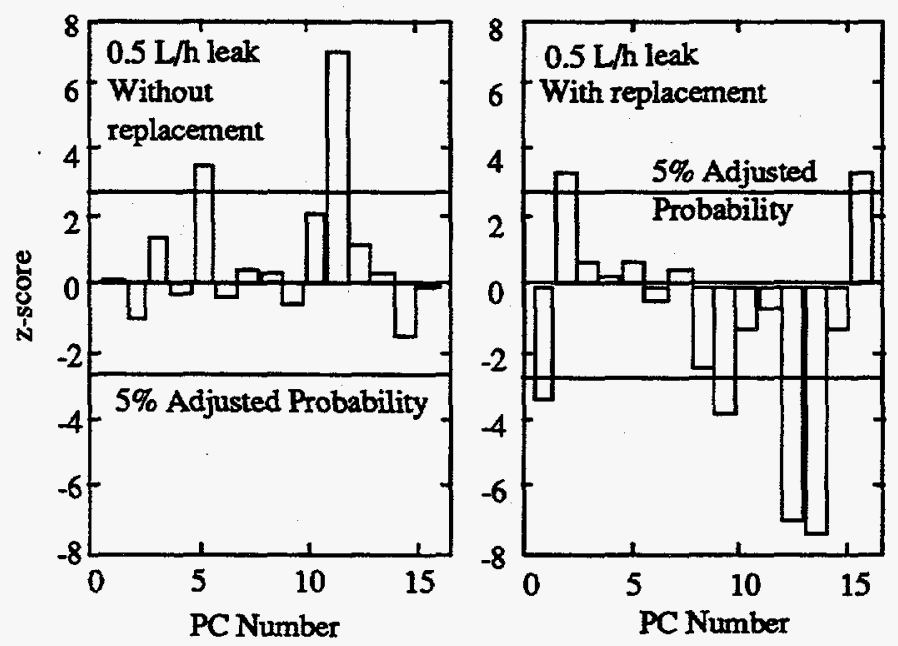

Fig. 2-7. The z-scores for the individual principal components corresponding to $0.5-\mathrm{L} / \mathrm{h}$ leaks with and without replacement of the lost volume.

Table 2-VIIa. Summary of Results for Leaks without Replacement: $z$-Scores

Leak

Variable

$\frac{\text { Leak }}{0.2 \mathrm{~L} / \mathrm{h} \quad 0.25 \mathrm{~L} / \mathrm{h} \quad 0.3 \mathrm{~L} / \mathrm{h} \quad 0.5 \mathrm{~L} / \mathrm{h}}$

Vol-2

$-2.43$

$-3.14$

$-3.85$

$-6.66$

$5 \%$ Critical

PC-5

1.37

1.72

2.07

3.47

2.72

PC-11

2.28

3.07

3.86

7.01

2.72

2.72

\begin{tabular}{lccccc}
\hline \hline Table 2-VIIb. & $\begin{array}{l}\text { Summary of Results for Leaks without Replacement: } \\
\text { Mahalanobis }\end{array}$ \\
\cline { 2 - 4 } & \multicolumn{5}{c}{ Leak } \\
Variable & $0.2 \mathrm{~L} / \mathrm{h}$ & $0.25 \mathrm{~L} / \mathrm{h}$ & $0.3 \mathrm{~L} / \mathrm{h}$ & $0.5 \mathrm{~L} / \mathrm{h}$ & $\begin{array}{c}\text { 5\% Critical } \\
\text { Value }\end{array}$ \\
$5 \mathrm{PCs}$ & 2.60 & 3.93 & 5.57 & 15.14 & 11.07 \\
$11 \mathrm{PCs}$ & 8.65 & 14.59 & 22.21 & 69.68 & 19.68 \\
$\begin{array}{l}\text { All 15 } \\
\text { Variables }\end{array}$ & 10.51 & 16.56 & 24.44 & 72.88 & 25.00 \\
\hline \hline
\end{tabular}


2. Leak with Replacement. This scenario is the same as the leak without replacement in terms of the mass of SNM lost, but it differs in that no volume change is observed because the volume lost is replaced with water. The loss is evidenced by a decrease in density and in the concentrations of nitric acid, plutonium, and uranium because of dilution with water.

The $z$-scores for the individual variables are plotted in Fig. $2-5$ together with the 5\% probability levels after adjusting for the fact that we are performing 15 tests. The $5 \%$ levels are exceeded by four of the measured variables: concentrations of plutonium in Tanks 2 and 3, concentration of uranium in Tank 2 , and density in Tank 2 . The $z$-score of -7.4 for concentration of plutonium in Tank 2 is greater than the value of -6.7 for volume in Tank 2 under the no replacement scenario.

Mahalanobis distances are plotted in Fig. 2-6 together with the 5\% chi-squared probabilities corresponding to from 1 to 15 degrees of freedom. The $M d s$ exceed values of the test statistic for all values, with principal components 12 and 13 being especially influential on the final distance. The magnitude of the individual principal components is further demonstrated in Fig. 2-7 where principal components $1,2,9,12,13$, and 15 all exceed the 5\% level. (They exceed the $1 \%$ level as well.)

Results for all the variables for the 0.5-L/h leak are summarized in Table 2-VII. Results for the other simulations for the leak with replacement scenario are summarized in Table 2-IXa and Table 2-IXb. As for the leak without replacement, the $0.25-\mathrm{L} / \mathrm{h}$ leak is the lowest level detected at the $5 \%$ probability level. It is detected by the plutonium concentration residual in Tank 2 as well as by individual principal components 12 and 13 and by the Mahalanobis distance. According to individual $z$-scores, principal component 13 and plutonium are equally sensitive tests for the fault with a probability of about $0.45 \%$. A chi-squared probability of about $1 \%$ corresponds to the Mahalanobis distance of 36.0 for 15 degrees of freedom. Thus, as for the leak-without-replacement scenario, the univariate tests are more sensitive than the multivariate tests. The fact that principal component 13 is a slightly more sensitive test than is plutonium lends some support to a multivariate approach, but it is not substantial for these scenarios. 
Table 2-VIII. 0.5 L/h Leak with Replacement - Summary of Results on Individual Variablesa

\begin{tabular}{llllll}
\hline Variable & \multicolumn{1}{c}{$\begin{array}{c}\text { Predicted } \\
\text { Value }\end{array}$} & $\begin{array}{c}\text { Measured } \\
\text { Value }\end{array}$ & Residual & z-score & \multicolumn{1}{c}{$s_{r}$} \\
\hline $\mathrm{Vol}_{1}$ & 100.4437 & 100.7744 & 0.3308 & 0.4425 & 0.7475 \\
$\mathrm{Vol}_{2}$ & 152.0864 & 152.3696 & 0.2832 & 0.4043 & 0.7006 \\
$\mathrm{Vol}_{3}$ & 104.5693 & 104.4166 & -0.1528 & -0.3601 & 0.4242 \\
$\mathrm{HNO}_{3_{1}}$ & 5.1357 & 5.1127 & -0.0230 & -0.1840 & 0.1250 \\
$\mathrm{HNO}_{3}$ & $\mathbf{5 . 0 3 9 0}$ & 4.8708 & $-\mathbf{0 . 1 6 8 2}$ & $\mathbf{- 2 . 4 4 5 6}$ & $\mathbf{0 . 0 6 8 8}$ \\
$\mathrm{HNO}_{3_{3}}$ & 4.8312 & 4.7898 & -0.0413 & -0.3080 & 0.1342 \\
$\mathrm{Pu}_{1}$ & 0.8221 & 0.8209 & -0.0012 & -0.2035 & $5.65 \mathrm{e}-3$ \\
$\mathrm{Pu}_{2}$ & $\mathbf{0 . 8 1 9 2}$ & $\mathbf{0 . 7 9 9 2}$ & $\mathbf{- 0 . 0 1 9 9}$ & $-\mathbf{- 7 . 4 0 0 1}$ & $\mathbf{2 . 6 7 e - 3}$ \\
$\mathrm{Pu}_{3}$ & $\mathbf{0 . 4 1 0 0}$ & $\mathbf{0 . 4 0 3 7}$ & $\mathbf{- 0 . 0 0 6 3}$ & $\mathbf{- 3 . 3 2 4 0}$ & $\mathbf{1 . 8 8 e - 3}$ \\
$\mathrm{U}_{1}$ & 0.0196 & 0.0195 & -0.0001 & -0.3804 & $2.53 \mathrm{e}-4$ \\
$\mathrm{U}_{2}$ & $\mathbf{0 . 0 1 9 3}$ & $\mathbf{0 . 0 1 8 8}$ & $\mathbf{- 0 . 0 0 0 5}$ & $\mathbf{- 4 . 2 9 8 0}$ & $\mathbf{1 . 2 3 e - 4}$ \\
$\mathrm{U}_{3}$ & $\mathbf{0 . 0 0 9 6}$ & 0.0095 & -0.0001 & -2.2601 & $6.02 \mathrm{e}-5$ \\
$\rho_{1}$ & 1.4667 & 1.4664 & -0.0003 & -0.0519 & $5.26 \mathrm{e}-3$ \\
$\mathrm{P}_{2}$ & $\mathbf{1 . 4 6 2 4}$ & $\mathbf{1 . 4 5 0 3}$ & $\mathbf{- 0 . 0 1 2 1}$ & $\mathbf{- 3 . 5 4 0 4}$ & $\mathbf{3 . 4 2 e}-3$ \\
$\mathrm{P}_{3}$ & 1.3043 & 1.2997 & -0.0046 & -0.9530 & $4.87 \mathrm{e}-3$ \\
\hline aBold indicates exceeded 5\% critical value. & & & & \\
\hline \hline
\end{tabular}

\section{Three-Tank-Problem Results Summary}

Univariate tests on individual variables and on individual principal components were equally effective at detecting the losses of material. Because the principal components are linear combinations of individual variables they might be expected to provide more sensitive detection of outliers or faults for situations where two or more correlated variables are affected by a fault. For individual principal components this was not observed for the present system. The multivariate tests using the Mahalanobis distances were never as sensitive as the univariate tests. The leak with replacement scenario was detected with slightly more sensitivity than was the leak without replacement. This is undoubtedly because replacing the lost volume with water affects four variables: density and concentrations of nitric acid, plutonium, and uranium. In contrast, for the leak without replacement only one variable, volume, is affected. 


\begin{tabular}{|c|c|c|c|c|}
\hline \multirow[t]{2}{*}{ Table 2-IXa. } & \multicolumn{4}{|c|}{$\begin{array}{l}\text { Summary of Results for Leaks with Replacement: } \\
z-\text { Scores }\end{array}$} \\
\hline & \multicolumn{3}{|c|}{ Leak } & \multirow{2}{*}{$\begin{array}{l}\text { 5\% Critical } \\
\text { Value }\end{array}$} \\
\hline Variable & $0.2 \mathrm{~L} / \mathrm{h}$ & $0.25 \mathrm{~L} / \mathrm{h}$ & $0.5 \mathrm{~L} / \mathrm{h}$ & \\
\hline Pu-2 & -2.6 & -3.4 & -7.4 & 2.72 \\
\hline $\mathrm{U}-2$ & -1.8 & -2.2 & -4.3 & 2.72 \\
\hline$\rho-2$ & -1.4 & -1.7 & -3.5 & 2.72 \\
\hline PC-1 & -1.5 & -1.9 & -3.7 & 2.72 \\
\hline PC-2 & 1.2 & 1.6 & 3.3 & 2.72 \\
\hline PC-9 & -1.8 & -2.1 & -3.8 & 2.72 \\
\hline PC-12 & -2.2 & -3.0 & -7.0 & 2.72 \\
\hline PC-13 & -2.6 & -3.4 & -7.3 & 2.72 \\
\hline PC-15 & 0.8 & 1.2 & 3.3 & 2.72 \\
\hline \multicolumn{5}{|c|}{$\begin{array}{c}\text { Table 2-IXb. Summary of Mahalanobis Distances for All } \\
\text { Variables for Leaks with Replacement }\end{array}$} \\
\hline & \multicolumn{3}{|c|}{ Leak } & \\
\hline Variable & $0.2 \mathrm{~L} / \mathrm{h}$ & $0.25 \mathrm{~L} / \mathrm{h}$ & $0.5 \mathrm{~L} / \mathrm{h}$ & $\begin{array}{l}5 \% \text { Critical } \\
\text { Value }\end{array}$ \\
\hline $\begin{array}{l}\text { All } 15 \\
\text { Variables }\end{array}$ & 21.8 & 36.0 & 163 & 25.00 \\
\hline
\end{tabular}

\section{SUMMARY AND CONCLUSIONS}

Variable estimates from process models and measurements are expected to be available in future nuclear reprocessing plants. The continued development and eventual implementation of fault detection and diagnosis methods are probably inevitable given the dual concerns of safety and economic competitiveness. Developments in this field appear to offer significant benefits. The work is well thought out and rigorous and has a solid chemical engineering and statistical basis. It should have a positive impact on the future efficiency and safety of chemical processing plants. As with most theoretical R\&D, a lack of examples of practical applications is a serious limitation of the published work. However, it is our understanding, based on contacts associated with the chemical industry, that many progressive industries are developing proprietary process control and fault detection programs.

The availability of information from these diagnostic tools will provide a large amount of data that could provide information for safeguards and materials accountancy. An important 
question from a safeguards and accountability perspective becomes one of how this data can be used to improve the efficiency of safeguards and perhaps decrease the cost. That was the main reason for this study.

We have emphasized an analytical approach to fault detection whereby a model is used to calculate predicted values for variables that are compared to measured values. If the process is too complex or poorly understood for modeling based on fundamentals alone, empirical models may be used. If an empirical approach is used, the level of modeling detail can be made appropriate to the desired task. After steady state is attained, linear models designed to model deviations from steady state are often used. These considerably simplify the modeling problem. A major advantage of the approach combining fundamental modeling with measurements is that it attempts to take advantage of everything we know about the system we are working with. The redundancies provided by either approach give independent estimates of the same or functionally related process output variables that can be compared to one another. Thus, the most sensitive and powerful statistical hypothesis testing procedures can be directly applied to the residual differences obtained without having to resort to less-well-developed pattern recognition methods such as neural nets. If the data prove to be highly correlated, measures such as the Mahalanobis distance account for the correlations; other methods, such as principal components analysis, may allow the selection of linear combinations of variables with improved sensitivity for specific kinds of faults as demonstrated for the leak with replacement scenario. Principal components should be useful for modeling specific faults in a system where the residuals are highly correlated.

Development of the process mathematical model is one of the most difficult problems preventing implementation of model-based process fault detection for large reprocessing plants. This is despite the fact that many chemical systems can be modeled using either fundamental principles or empirical models or both. We believe that the most useful systems will involve a combination of qualitative (e.g., expert systems or fuzzy logic) and quantitative (mathematical models of unit processes) approaches. Simulation models, such as the facility simulation model FACSIM being developed at Los Alamos for the simulation of nuclear facilities, may have application for fault detection.

Process knowledge through process modeling combined with process information through process measurements is applicable to safeguards. Additional process measurements of SNM alone can be shown to improve our ability to detect material diversions. Measurements of correlated process variables can only improve this capability and, as demonstrated by our three-tank simulation, use of a model providing redundant estimation of measured process variables provides fault detection in real time. 
We recommend that efforts in this area be continued. As mentioned above, the primary limitation of the published work on fault detection is a lack of demonstrated applications. We believe that a follow-on program should have as its main purpose the development, implementation, and evaluation of a process modeling and fault detection system based on simulated data from a real reprocessing plant. The system should then be tested at such a plant.

Another area that is of considerable interest is to further evaluate the three-tank system under different fault scenarios and to add more complicated unit processes, e.g., pulsed columns, to this system and to compare the results with plant data if possible. Also for the three-tank system, we believe that the model predictions might be generated using a linear state equation in the classical Kalman Filter, development of a Kalman Filter approach to this problem would be an interesting and potentially useful comparison.

Finally, based on preliminary results from the three-tank simulation, we propose to initiate a study involving multivariate time series. It is common to reduce multivariate time series to univariate time series by using simple linear combinations of the individual variables. It could be useful for safeguards to identify cases where analysis of time series as multivariate data is advantageous over performing the analysis on linear combinations of the univariate observations.

\section{REFERENCES}

2-1. Paul M. Frank, "Fault Diagnosis in Dynamic Systems Using Analytical and Knowledgebased Redundancy-A Survey and Some New Results," Automatica 26, 459-474 (1990).

2-2. Paul M Frank and Ralf Seliger, "Fault Detection and Isolation in Automatic Processes," Control and Dynamic Systems 49, 241-287 (1991).

2-3. Vic Barnett and Toby Lewis, Outliers in Statistical Data, 2nd ed. (John Wiley and Sons, New York, 1984).

2-4. Richard Johnson and Dean Wichern, Applied Multivariate Statistical Analysis (Prentice Hall, New Jersey, 1988).

2-5. Chen-Shan Kao, Ajit C. Tamhaniand, Richard S. H. Mah, "Gross Error Detection in Serially Correlated Data," Industrial Engineering Chemical Research 29, 1004-1012 (1990).

2-6. Janos J. Gertler, "Survey of Model-Based Failure Detection and Isolation in Complex Plants," IEEE Control Systems Magazine (December 3-11, 1988).

2-7. Ajit C. Tamhane and Richard S. H. Mah, "Data Reconciliation and Gross Error Detection in Chemical Process Networks," Technometrics 27, 409-422 (1985).

2-8. Richard J. Beckman and R. Dennis Cook, "Outliers," Technometrics 25, 119-149 (1983). 
2-9. Martin A. Stapanian, Forest C. Garner, Kirk E. Fitzgerald, George T. Flatman, and Evan J. Englund, "Properties of Two Tests for Outliers in Multivariate Data," Communications in Statistics - Simulation and Computation 20, 667-687 (1991).

2-10. Peter J. Rousseeuw and Bert C. van Zomeren, "Unmasking Multivariate Outliers and Leverage Points" Journal of the American Statistical Association 85, 633-639 (1990).

2-11. R. Gnanadesikan, Methods for Statistical Data Analysis of Multivariate Observations (John Wiley and Sons, New York, 1978), pp. 259ff.

2-12. Tormod Naes, "Leverage and Influence Measures for Principal Components Regression," Chemometrics and Intelligent Laboratory Instrumentation 5, 155-168 (1989).

2-13. J. Edward Jackson, "Multivariate Quality Control," Communications in Statistics Theoretical Methods 14, 2657-2688 (1985).

2-14. S. Schoof, "Spent Nuclear Fuel Reprocessing: Chemometrical Treatment of Input Analytical Data," ESARDA Bulletin No. 18, 6-11 (1990). 


\section{CHAPTER 3}

\section{NONLINEAR TIME SERIES ANALYSIS APPLIED TO ANOMALY DETECTION IN NEAR-REAL-TIME ACCOUNTING DATA}

\section{INTRODUCTION}

In this chapter we consider the application of nonlinear time series techniques to monitor and analyze univariate data from highly automated nuclear fuel reprocessing plants. The major goal is to determine whether recent advances in nonlinear time series analysis might help detect losses of nuclear material. Another goal is to better understand the physical processes that generate the univariate data under the assumption that no nuclear material has been lost or diverted.

The main contents of this chapter are a literature review and a documentation of the data analyses that have been performed. Our goal is to study the possibility of applying methods recently put forth in nonlinear time series literature to analyze NRTA data from fuel reprocessing plants. Typically, more data is required for the analysis of nonlinear systems than for linear systems.

Although today's fuel reprocessing plants normally perform material balances (MBs) only infrequently, we anticipate that future plants will have the capacity to monitor much more frequently. With the higher rate of data collection, it will be possible to apply some of the recent methods of detecting nonlinearity and fitting nonlinear models.

The usual analysis of a sequence of $\mathrm{MBs}$ assumes that the sequence has a multivariate normal distribution. The variance-covariance matrix of the sequence depends on the variance of the measurement process and is rather tedious to estimate. The multivariate normal (MVN) assumption should not seriously affect the statistical decisions, but the availability of very long sequences of estimated losses might make it advantageous to apply more involved data analysis, such as nonlinear modeling. For example, suppose that a functional form for how the observed MB depends on previous MBs could be estimated from the data. The estimated functional form might provide a better understanding of the physical processes generating the data as well as detect losses better than the present approach. The present approach assumes multivariate normality and therefore implicitly makes the restrictive assumption that the functional form is linear.

Consider a sequence of observations $X_{1}, X_{2}, \ldots, X_{t}$, indexed by the observation order. Such a sequence is commonly referred to as a time series. The observation $X_{5}$ could, for example, denote the $\mathrm{MB}$ on the fifth day of operation. It is typical for an observed time series to exhibit serial correlation. That is, observations $X_{t}$ and $X_{t+1}$ might tend to be more alike 
(positive correlation) or more different (negative correlation) than observations $X_{t}$ and $X_{t+20}$. Therefore, any time series model should be able to accommodate serial correlation.

In general, the analysis of time series assumes that the observation $X_{t}$ is somehow affected by some subset of all previous observations. For example, the lag 1 autoregressive [AR(1)] model is $X_{t}=a_{1} X_{t-1}+\varepsilon_{t}$, where $\left|a_{1}\right|<1$ and $\varepsilon_{t}$ has a normal (Gaussian) distribution with mean 0 and variance $\sigma^{2}$, which we denote $\varepsilon_{t} \sim N\left(0, \sigma^{2}\right)$. The error term $\varepsilon_{t}$ can include certain types of both model misspecification and observational error. The lag $2 \mathrm{AR}$ model is $X_{t}$ $=a_{1} X_{t-1}+a_{2} X_{t-2}+\varepsilon_{2}$, and so on, up to lag $p$. A second class of models is the moving average (MA) models. The MA(1) model is $X_{t}=\varepsilon_{t}+b_{1} \varepsilon_{t-1}$, with $\left|b_{1}\right|<1, \varepsilon_{t} \sim N\left(0, \sigma^{2}\right)$, and again, the lag can be extended, say to lag $q$.

Linear Gaussian models are a successful class of models that exhibit serial correlation. Linear Gaussian models combine both the AR and MA models and can handle long-term trends. The effect of long-term trends in the data swamps the more local effect that observations may have on their successors in the sequence. Therefore, we assume that any long-term trends, such as a rising level of the series, have been handled in the usual manner, by differencing the series. The new series of, say, first differences is $D_{1}=X_{2}-X_{1}, D_{2}=X_{3}-X_{2}, \ldots$.

Linear Gaussian models have dominated the statistical analysis of time series since Yule's AR models in 1927. Reference 3-1 is a good introductory text for the application of linear Gaussian models to time series. Although it is not our intent to review linear Gaussian models in any detail, we do intend to compare the performances of linear and nonlinear analyses. We also propose to compare the performance of the current method of analyzing time series from nuclear fuel reprocessing facilities in a later report. We discuss the current method in Sec. II. The current method implicitly assumes a linear model but does not quite fit the AR or MA paradigm.

There has been much recent interest in nonlinear time series analysis and modeling, which is partly attributable to the curious phenomenon known as chaos. Chaos is often loosely defined as sensitivity to initial conditions, and can be present in nonlinear, but not in linear, time series models. We will introduce the notation by modifying the notation used for linear models.

Write the usual Gaussian linear model $(\operatorname{ARMA}(p, q))$ for the time series $\left(X_{1}, X_{2}, \ldots\right.$, $X_{n}$ ) as follows:

$$
X_{t}=a_{0}+\sum_{j=1}^{p} a_{j} X_{t-j}+\sum_{j=0}^{q} b_{j} \varepsilon_{t-j}
$$


where the $a_{j}$ and $b_{j}$ are real constants, the $\varepsilon_{t-j}$ are independently and identically distributed (iid) $N\left(0, \sigma^{2}\right)$ random variables, and $t \in\{1,2, \ldots, n\}$. Note that $\operatorname{ARMA}(p, q)$ models combine $\mathrm{AR}(p)$ and $\mathrm{MA}(q)$ models.

Gaussian linear models will undoubtedly continue to be applied in many areas of research, sometimes with good results. The model (3-1) can be expressed as

$$
X_{t}=f\left(X_{t-1}, X_{t-2}, \ldots, X_{t-p}\right)+\varepsilon_{t}
$$

for a suitably defined linear function $f: \Re^{p} \rightarrow \Re$, and for $\varepsilon_{t}=\varepsilon_{t}$; a suitably defined function of $\varepsilon_{t}, \varepsilon_{t-1}, \ldots, \varepsilon_{t-q}$. For ease of notation, Eq. (3-2) has been written using $\varepsilon_{t}$ rather than $\varepsilon_{t}^{\prime}$.

There are two common ways to generalize the model given by Eq. (3-2). The distribution of the error term $\varepsilon_{t}$ could be non-Gaussian and the function $f$ could be nonlinear. Our primary interest is in nonlinear $f$.

Certain nonlinear $f$ have trajectories whose properties have attracted much recent attention from statisticians, physicists, and applied mathematicians. A trajectory is a sequence of state vectors, for $t=\{d+1, d+2, \ldots, n\}$, where the state vector $\mathbf{X}_{t}=\left(X_{t} X_{t-1}, \ldots, X_{t-d}\right)$. The parameter $d$ is known as the embedding dimension or simply the lag. In Eq. (3-2), the lag is denoted $p$, but to be consistent with most of the nonlinear time series literature, we will use $d$ rather than $p$ for the lag.

The logistic map, with $0<a \leq 4$ and the initial value $x_{0}$ in $(0,1)$, is a well-known example:

$$
x_{t+1}=a x_{t}\left(1-x_{t}\right) \text { for } t \text { in } T=\{0,1,2, \ldots, n-1\} .
$$

Note that $x_{t} \in(0,1)$ for all $t$, provided we ignore cases such as $x_{0}=0.5$ and $a=4$, which implies that $x_{1}=x_{2}=\ldots=1$. Also regarding notation, we use upper case letters for random variables and lower case letters for deterministic variables or for realized values of random variables. The distinction should be clear from the context. Compare Eqs. (3-2) and (3-3). Equation (3-3) is deterministic, so we use the lower case $x_{t+1}$.

In terms of model (3-2), in Eq. (3-3) we have set $\varepsilon_{t}=0$ for all $t, f(x)=a x(1-x)$, and the $\operatorname{lag} d=1$. One surprising feature of Eq. (3-3) is that for certain values of $a$, its deterministic trajectories appear to be random. In Fig. 3-1 we plot observations from the logistic map for $a=2.5$ and for $a=4$. For $a=2.5$, in Fig. 3-1a, the trajectory does not appear random, but for $a=4$, in Fig. 3-1b, the trajectory does appear random. See Sec. II for further discussion. Anyone familiar with deterministic methods used to generate pseudo-random numbers is already acquainted with the phenomenon whereby a deterministic difference equation results in trajectories that have properties usually described as random. 


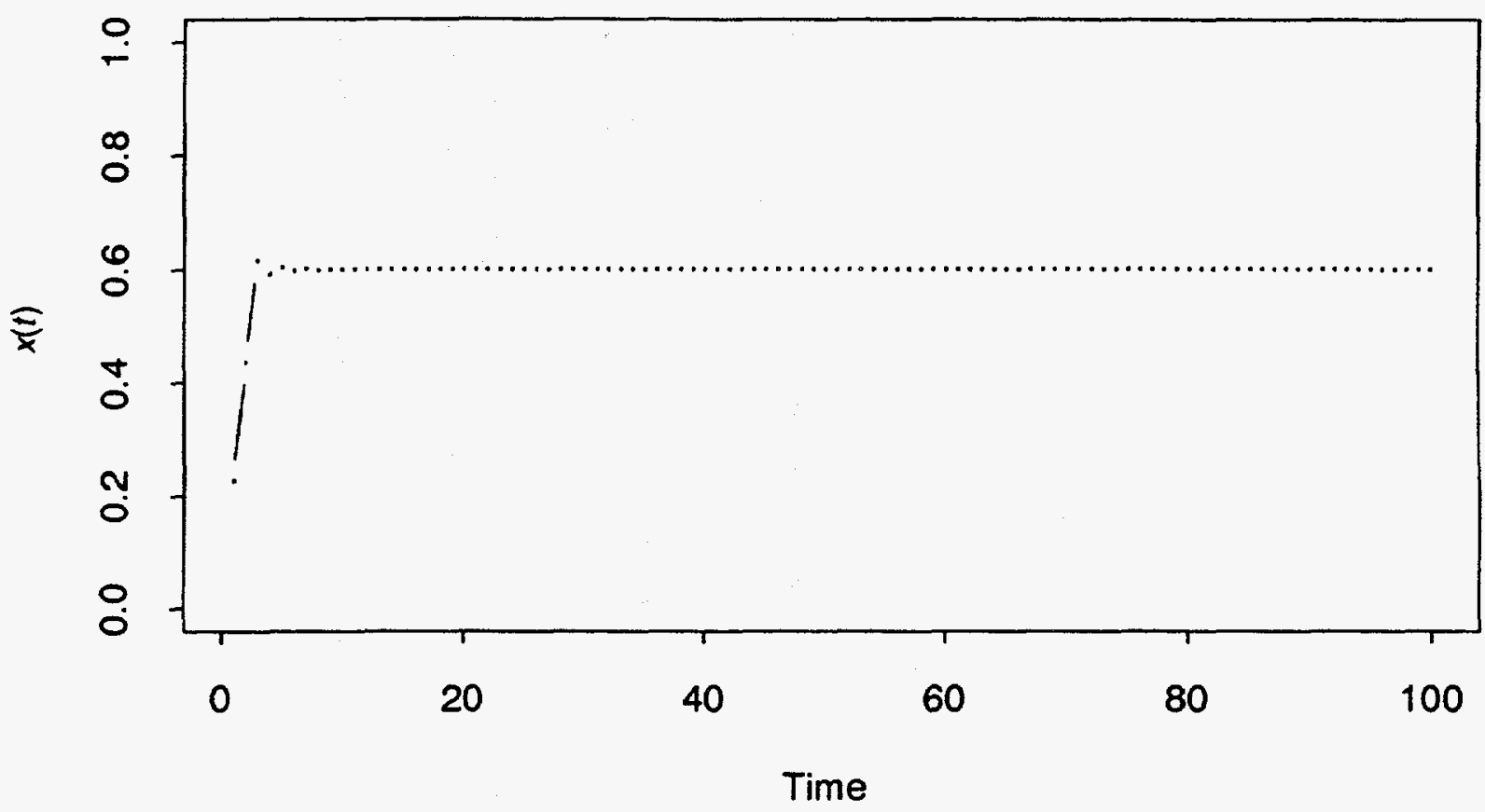

(a)

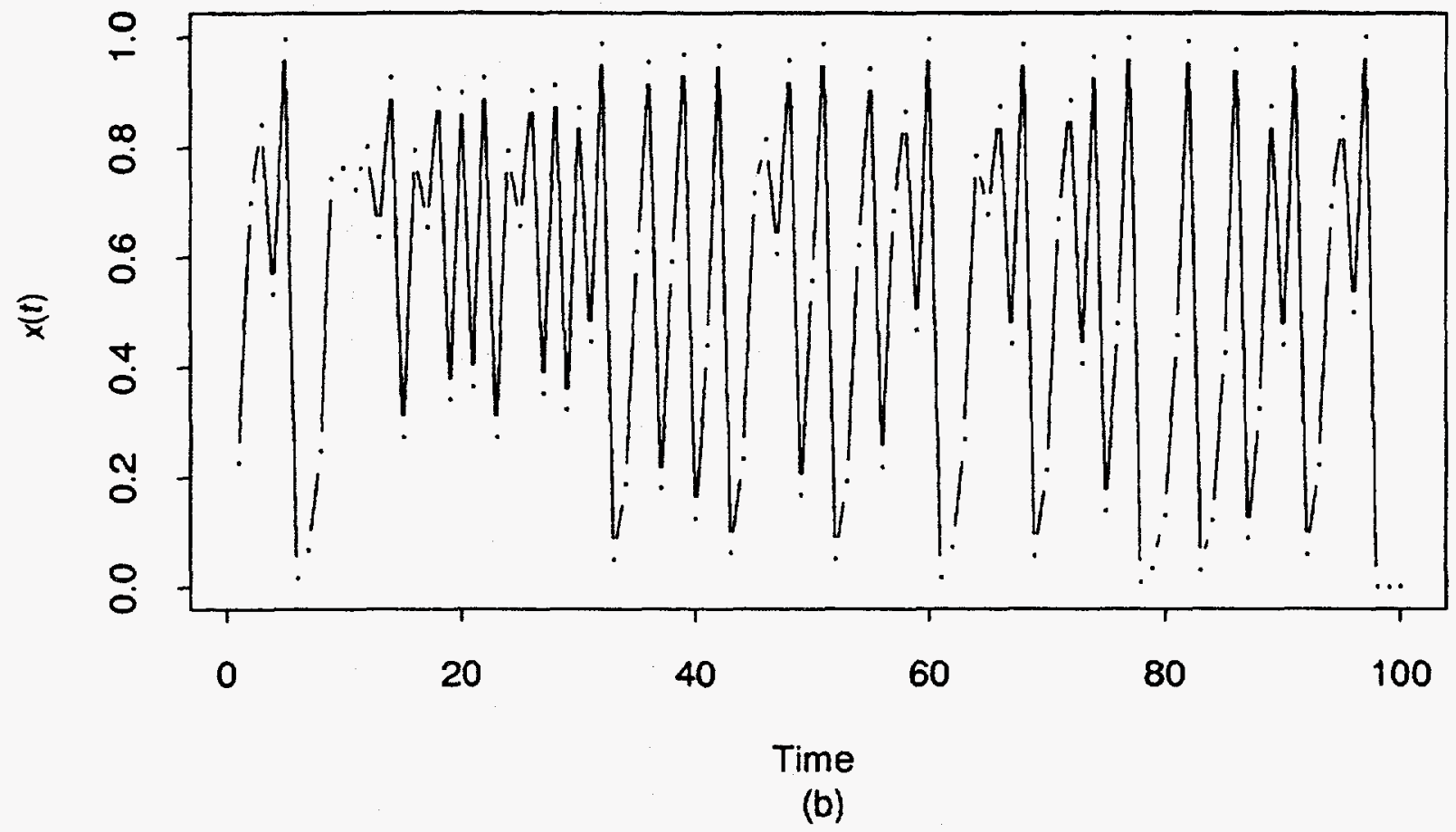

Fig. 3-1. The logistic map: (a) $a=2.5, x(1)=0.2256$; (b) $a=40, x(1)=0.2256$. 
Other features of Eq. (3-3) help illustrate some of the concepts of nonlinear time series that are reviewed in Sec. II. One goal is to understand and quantify the effect of modeling a nonlinear time series with a linear model. Another goal is to detect nonlinearity, even in the presence of the error term $\varepsilon_{t}$. In Sec. III, traditional methods of analyzing NRTA data are reviewed and discussed. A description of fundamental tools for detecting and modeling nonlinearity is given in Sec. IV, along with exemplary data analyses. In Sec. V we describe some additional new tools from the analysis of nonlinear time series that we have begun to test. In Sec. VI we present further data analyses with emphasis on whether nonlinear modeling can improve loss detection in NRTA data. A summary and possible benefits of adding nonlinear modeling are given in Sec. VII.

\section{NONLINEAR TIME SERIES}

The logistic map given in Eq. (3-3) can be used to illustrate two features that are sometimes present in nonlinear time series.

The first feature is sensitivity to initial conditions, as measured by Lyapunov exponents. In the case of the map given by Eq. (3-3), consider two distinct initial points, say $x_{0}$ and $x_{0}^{\prime}$. Write Eq. (3-3) as

$$
x_{t}=f\left(x_{t-1}\right)
$$

After $n$ iterations of the map $f$, denoted $f^{(n)}$, we have $x_{n}$ and $x_{n}^{\prime}$ corresponding to $x_{0}$ and $x^{\prime} 0$, respectively. Define the Lyapunov exponent

$$
\lambda\left(x_{0}\right)=\lim _{n \rightarrow \infty} \ln \left|\frac{d}{d x} f^{(n)}\left(x_{0}\right)\right|^{1 / n} .
$$

Provided some technical conditions hold, by an appeal to ergodic theory, $\lambda\left(x_{0}\right)$ does not depend on $x_{0}$. Assuming that $\lambda\left(x_{0}\right)$ does not depend on $x_{0}$, denote $\lambda\left(x_{0}\right)$ by $\lambda$. A good way to interpret Eq. (3-5) is recognize that Eq. (3-5) implies

$$
\left|x_{n}-x_{n}^{\prime}\right|=\mathrm{e}^{\lambda n}\left|x_{0}-x_{0}^{\prime}\right|
$$

Equation (3-6) is a reasonable approximation only for small values of $\lambda n$ because we assume that the map $f$ allows the map to remain bounded, yet the factor $\mathrm{e}^{\lambda n} \rightarrow \infty$ as $n \rightarrow \infty$ if $\lambda>0$. Suppose that the two initial points $x_{0}$ and $x_{0}^{\prime}$ are very close and that $\lambda>0$. Then, Eq. (3-6) implies that after $n$ iterations of the map, the points $x_{n}$ and $x_{n}^{\prime}$ that correspond to the two initial points, $x_{0}$ and $x_{0}^{\prime}$, will be quite separated because of the factor $\mathrm{e}^{\lambda n}$. 
For the logistic map with $a=4$, it can be shown that $\lambda=\ln 2>0$. Equation (3-6) implies that if $\lambda>0$ then iterations of the map $f$ diverge at an exponential rate, which is a rather extreme sensitivity to initial conditions. In the analysis of a time series, specification of which observation is the initial observation is arbitrary. Any of the observations could be regarded as the first observation. This idea can be used to detect nonlinearity in a time series. See Sec. IV for further discussion.

The second feature that Eq. (3-3) can illustrate is the unpredictability of chaotic processes. Suppose we are allowed to observe, without error, a realization of 500 observations of Eq. (3-3) with an unknown value of $a$. For $a \leq 1$, the realization will approach 0 . For $1 \leq a \leq$ 3 , the realization will approach the limit $1-1 / a$, but for large $a$, say $a=4$, the realization will appear at first glance to be random. Shown in Fig. 3-1 are the first 100 observations of the logistic map for two cases: $a=2.5$ and $a=4$. Note that for $a=2.5, x_{n} \rightarrow 0.6$, and for $a=4$, $x_{n}$ does not approach any limit, but rather, appears to be random.

If we are allowed to observe without error a realization of 500 observations of the map given in Eq. (3-3), then a pairwise scatterplot of $x_{n+1}$ versus $x_{n}$ would reveal the parabolic shape and reveal the value $a=4$. See Fig. 3-2b, in which $a=4$. However, if those 500 observations of Eq. (3-3) are made with error, we in effect have uncertainty in initial conditions, no matter which observation we treat as the initial observation. It turns out that any reasonable procedure to predict $x_{n}$ beyond the 500 observations will quickly appeal to the mean of the underlying ergodic distribution. ${ }^{3-2}$ For example, the prediction of $x_{510}$ based on the first 500 observations, each observed with error, would essentially be the sample mean of the 500 observations. We can use Fig. 3-2a to explain why as follows. We intend to show that unless $x_{500}$ is known exactly, estimates of, say, $x_{510}, x_{511}, \ldots$ might as well ignore the imprecisely known value of $x_{500}$, even if $a$ were known precisely. In Fig. 3-2a we show the differences $d_{1}$ $=x_{1}-x_{1}^{\prime}, d_{2}=x_{2}-x_{2}^{\prime}, \ldots, d_{100}=x_{100}-x_{100}^{\prime}$, where $x_{1}=0.22559$ and $x_{1}^{\prime}=0.2256$, with $a=4$. The point is that by about the 10th iterate of the logistic map, the differences $d_{10}, d_{11}, \ldots$ are large and unpredictable, even though $d_{1}=x_{1}-x_{1}^{\prime}=-0.00001$. The implication is that unless we know $x_{500}=0.2256$ exactly (and not, say, $x_{500}=0.22559$ ), then in predicting $x_{510}$ we might as well ignore the value $x_{500} \approx 0.2256$ and use the sample mean of the first 500 observations to predict observation $x_{510}$. This feature is similar to the behavior of forecasts from Gaussian linear models. Fairly quickly, the forecasts "decay" to the sample mean of the observed sequence.

We also point out from Fig. 3-1b, that for $a=4$, the "attractor" of the map is the unit interval $(0,1)$ provided $x_{0} \neq 0$ or 1 . An attractor can be thought of as the set of possible values of the map after many iterations. So for $a=4, x_{n}$ could be anywhere in the interval $[0,1]$. 


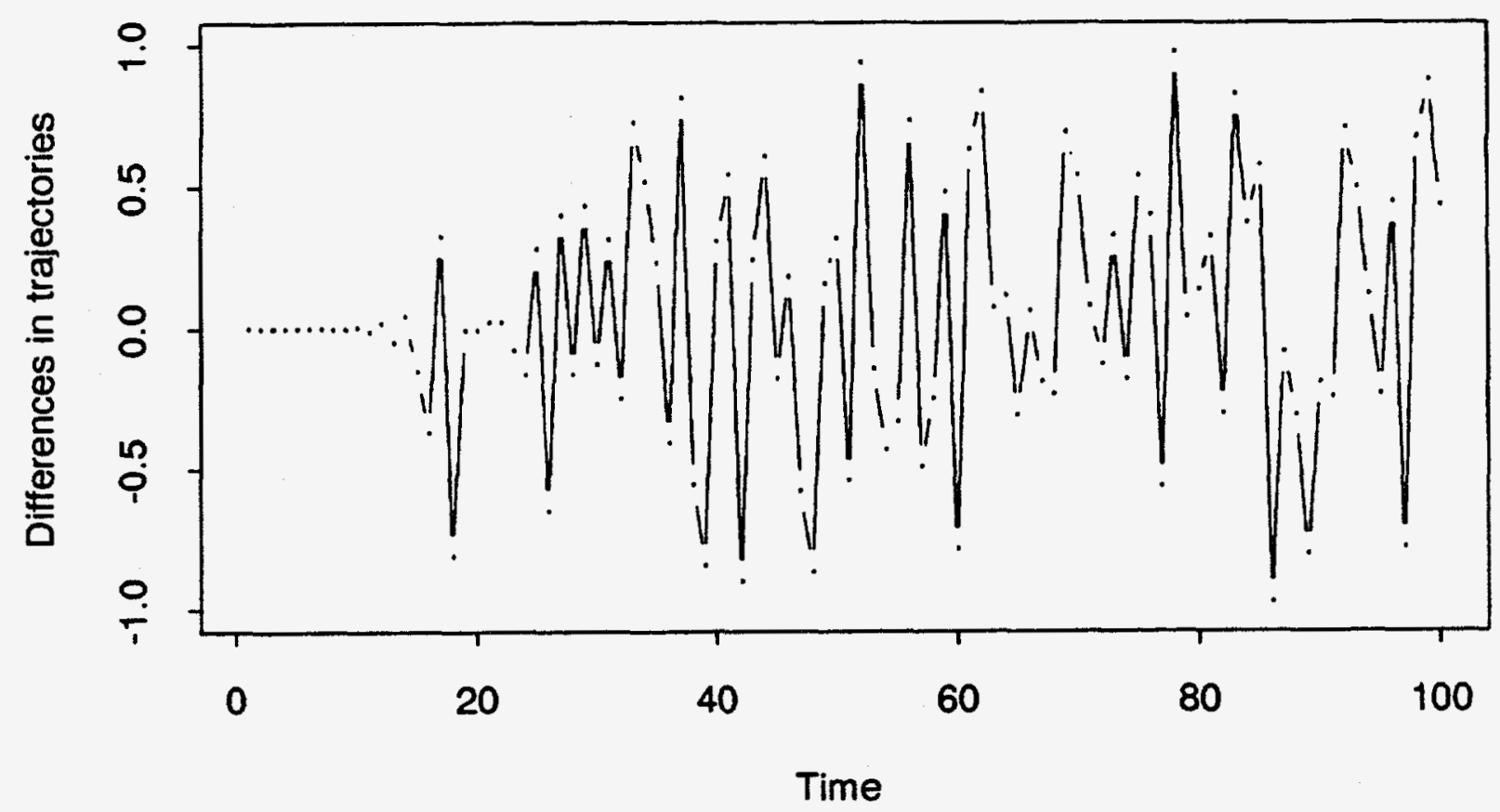

(a)

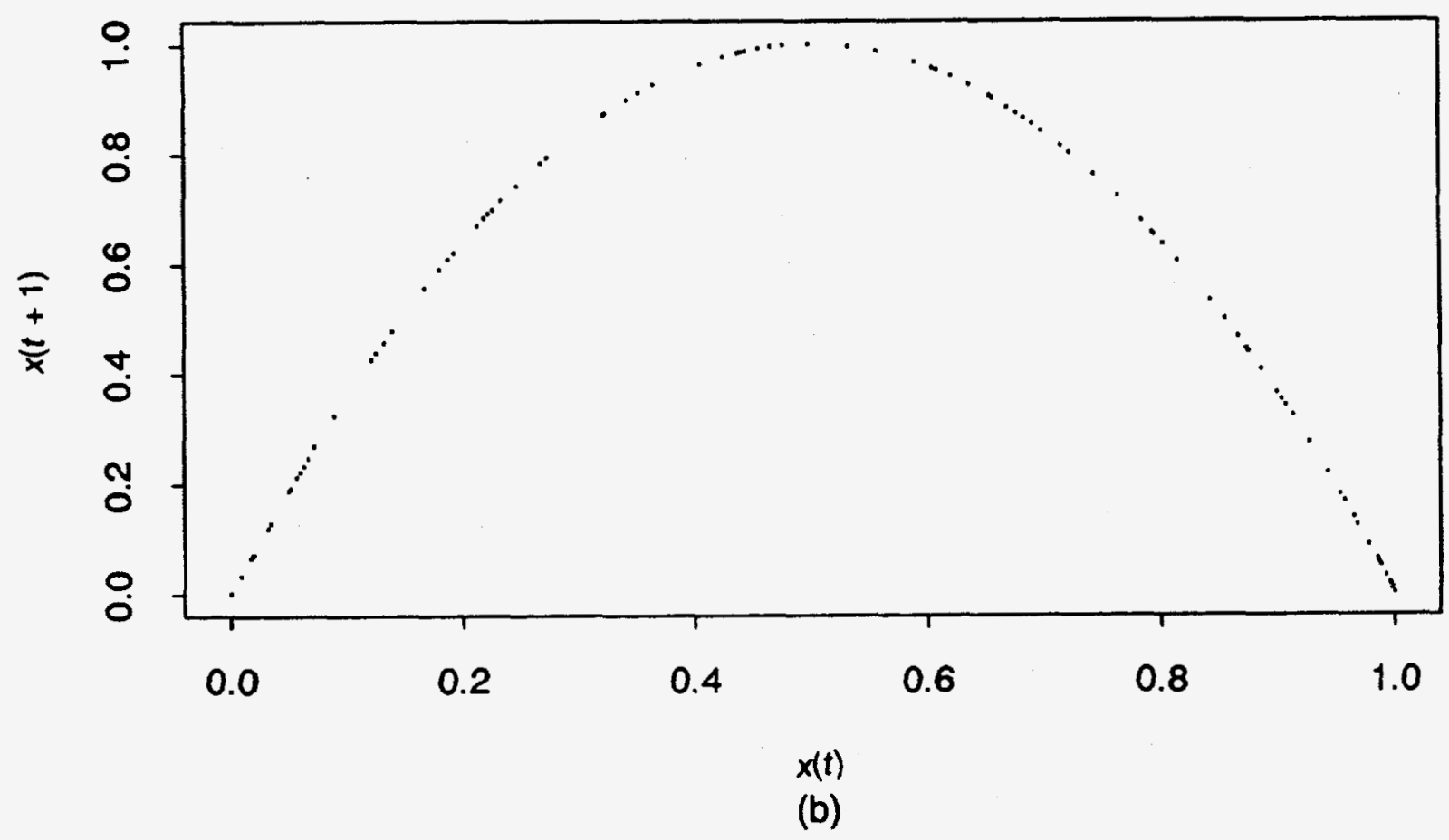

Fig. 3-2 The logistic map: (a) sensitivity to initial conditions; (b) $a=40, x(t+1)$ vs. $x(t)$. 
More formally, if the lag is $d$, then as $n \rightarrow \infty$, the $d$-vectors $\mathbf{y} n=\left(x_{n-d+1}, \ldots, x_{n}\right)$ approach a limiting set known as the attractor. An unusual feature of the attractor of some nonlinear maps is that they have non-integer dimension. We give one definition of dimension in the context of nonlinear time series in Sec. V. Non-integer dimension is a feature of fractals. An attractor having non-integer dimension (i.e., a fractal) is referred to as a strange attractor, and the nonlinear map is referred to as a chaotic map. We show an example (the Henon map) in Sec. V. This kind of phenomenon has given rise to characterizing nonlinear time series by the dimension of their attractors.

A second example ${ }^{3-3}$ that is useful for illustrating sensitivity to initial conditions is the binary shift map

$$
x_{t+1}=2 x_{t}(\bmod 1), t=1,2, \ldots
$$

where $x_{0} \in[0,1]$, and $x \bmod 1$ is the remainder when $x$ is divided by 1 . For example, if $x_{1}=0.6$, then according to Eq. (3-7), $x_{2}=1.2 \bmod 1=0.2$. Because $0 \leq x_{t} \leq 1$ for all $t$, the map is globally bounded. The map (3-7) admits an easy solution:

$$
x_{t}=2^{t} x_{0}(\bmod 1), t=1,2, \ldots
$$

Write $x_{0}$ in the binary expansion as $x_{0}=. k_{1} k_{2} \ldots$, where $k_{i} \in\{0,1\}$. For example, the binary representation of $1 / 2$ is 0.100000 . Because each application of the binary shift map (3-7) removes the leftmost digit, any two initial values $x_{0}$ and $x_{0}^{\prime}$ that are very close together, agreeing say, in the first $p$ positions, will after $p$ iterations be spread apart. The map (3-7) is therefore sensitive to initial conditions $\left(\lambda\left(X_{0}\right)=\ln 2\right.$, for any value of $\left.X_{0}\right)$, and so has the two features required for chaos: global boundedness and local instability. In Fig. 3-3a we show the first 50 observations of Eq. (3-7) with $x_{1}=0.789$. In Fig. 3-3b we plot $x_{t+1}$ versus $x_{t}$, and in Fig. 3-3c we plot $d_{1}=x_{1}-x_{1}^{\prime}, d_{2}=x_{2}-x_{2}^{\prime}, \ldots, x_{n}-x_{n}^{\prime}$, where $x_{1}=0.789$ and $x_{1}^{\prime}=$ 0.789001. Note in Fig. 3-3c, that after about 18 iterations of the binary shift map, the initially close values have separated.

It is not possible to make a blanket statement about the relative ease of predicting nonlinear time series versus predicting linear time series because the random error variance must be considered. In some cases, nonlinear time series forecasts have smaller variances than linear time series forecasts. In other cases, the opposite is true.

Probably, the interest in safeguards will not be in prediction, per se, but in quick detection of true losses. It is possible that loss detection can be improved by using nonlinear time series analysis instead of linear time series analysis. That would depend on whether the 


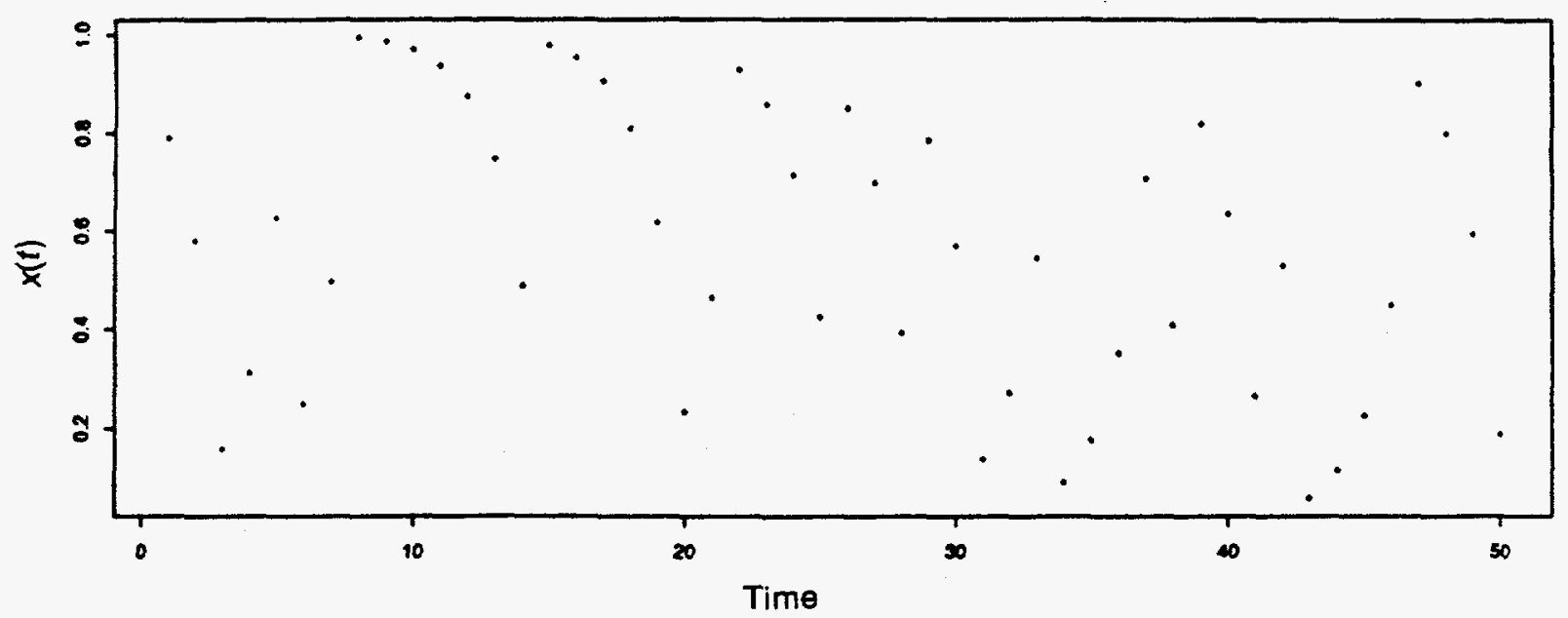

(a)

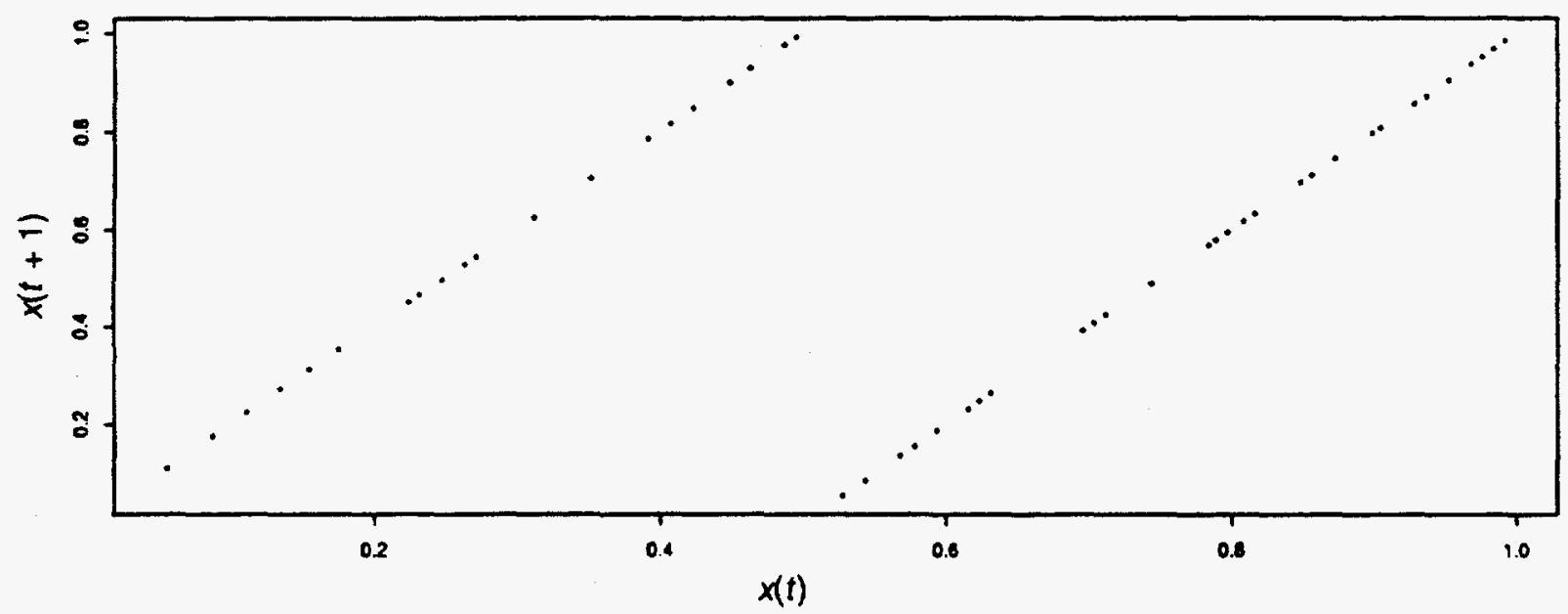

(b)

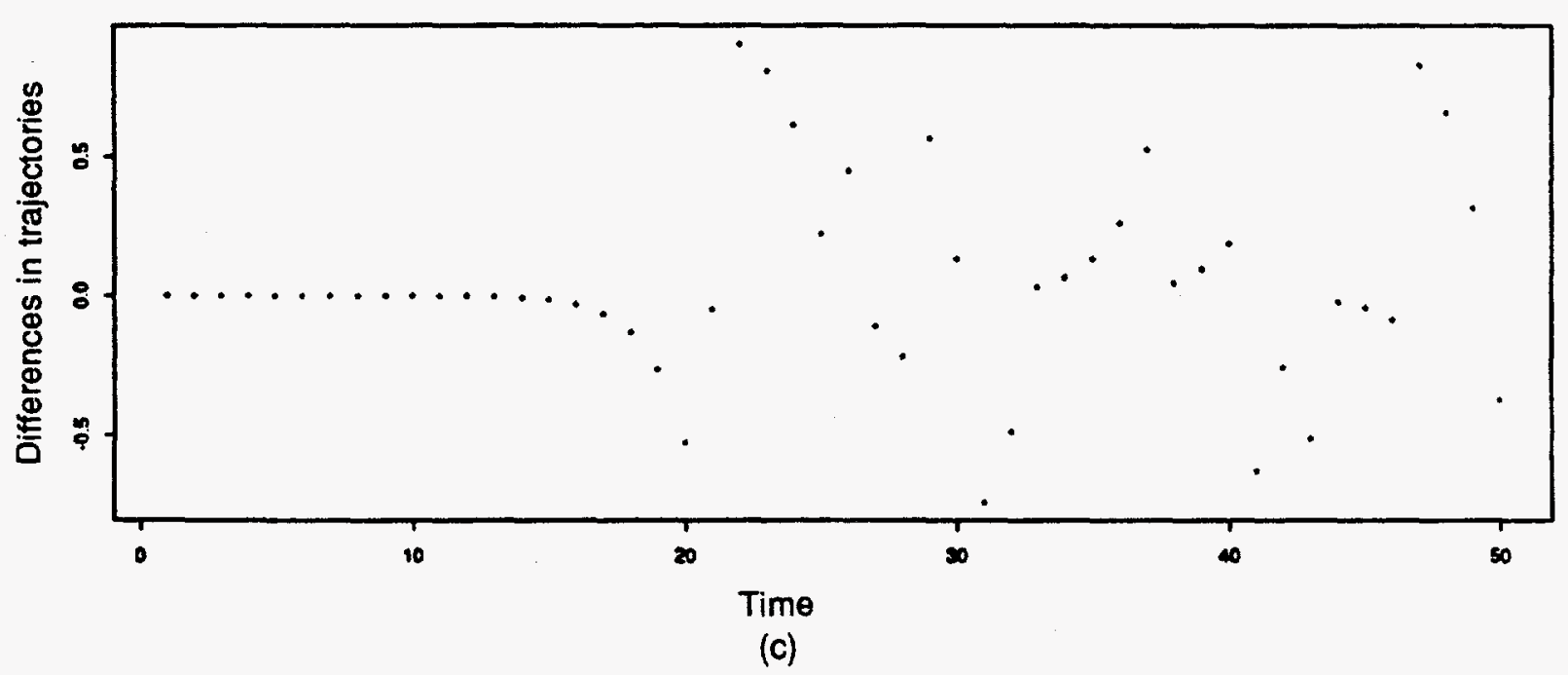

Fig. 3-3. The binary shift map: (a) 50 observations ; (b) $x(t+1)$ vs. $x(t)$; (c) sensitivity to initial conditions. 
observed MB at time $n+1$ actually is a nonlinear function of the observed MB at time $n$ under the assumption of no material loss. We consider this issue in more detail in Sec. IV.

A second interest in safeguards is simply to better understand the processes generating the data. A good first step in that direction is empirical modeling. And, because of the large amount of data expected, it is reasonable to drop the assumption that the mean of $x_{t}$ is a linear function of some subset of the prior observations and hence apply nonparametric or semiparametric estimation tools from nonlinear time series literature.

Having made these last few remarks, we must add that it is quite possible that no reasonable physical model for the data from a fuel reprocessing plant suggests that nonlinear modeling is actually needed. Nevertheless, we feel that empirical data analysis should accompany the traditional analysis of MB sequences and that the recent advances in the analysis of nonlinear time series should be considered by the safeguards community.

Several specific forms of nonlinear $f$ have been studied in the literature. Examples include random coefficient AR models and threshold AR models. ${ }^{3-3,3-4}$ This section has focused on two of the features of some nonlinear models. Research in nonlinear modeling has been motivated partly by the very surprising behavior, such as sensitivity to initial conditions and unpredictability, of some nonlinear maps.

The point of view adopted here is somewhat different. The view is that it should be possible to estimate certain properties of the unknown nonlinear function $f$ in Eq. (3-2) by observing a sufficiently long time series. This is more the usual statistical point of view, whereby we observe a sequence and build models of what might have generated it. For example, the conditional mean of $X_{t}$ given $X_{1}, X_{2}, \ldots, X_{t-1}$, is one property of $f$ that is useful to know for the purpose of making a statistical decision about whether $X_{t}$ is too large under the assumption of no loss of material. We therefore emphasize estimation of the conditional mean in Sec. IV. If it turned out that any process generated by the estimated $f$ would be a chaotic process, then we would certainly seek better physical understanding of the process generating the data.

We conclude Sec. II by describing the type of time series we will consider. We intend for Eq. (3-2) to accommodate certain nonlinear functions $f$, but some restrictions apply. First, suppose the true values of a time series are denoted by $X_{i}$ but that we observe $Y_{i}=X_{i}+\varepsilon_{i}$, where $\varepsilon_{i}$ is the observation error. We might then write Eq. (3-2) as

$$
Y t=f\left(Y_{t-1}-\varepsilon_{t-1}, Y_{t-2}-\varepsilon_{t-2}, \ldots, Y_{t-d}-\varepsilon_{t-1}\right)+\varepsilon_{t}
$$

Recall that we are using $d$ rather than $p$ for the lag to be consistent with most of the nonlinear time series literature. Equation (3-9) implies that the errors propagate through the system 
so we have added the complication that is implicit in an "errors-in-variables" model. An example of how the errors-in-variables issue arises is the case of regressing a response variable $y$ on a predictor variable $x$ when $x$ is measured with error. If there were no error in $x$, we would have simple linear regression. The measurement error in $x$ complicates the analysis, more than at first might be expected. See Ref. 3-5 for an example of how this system noise complicates a particular estimation procedure.

The distinction between observational and system noise is a concern in some of our simulated data. For example, if in the logistic map given by Eq. (3-3), system noise affected the $x_{t}$, then whenever $x_{t}>1$ the map would diverge to $-\infty$. So, in the case of the logistic map, we simulate only observational noise by generating a sequence without any observation error or system error, according to Eq. (3-3), and then generate an observed sequence by adding an observational error to each of the true values in the sequence.

From the viewpoint of nonlinear estimation for real data, the distinction between observational and system noise is a concern if the goal is to estimate $f$ in Eq. (3-9). However, our main goal is to predict $X_{t}$ given its relevant predecessors, and in that case the distinction between observational and system noise is not an issue. Therefore, the process generating any real data we consider can include both systematic and observational error.

Second, we recognize two very different cases of interest. In one case, we can assume that the lag $d$ (recall that we restrict attention to fixed lags) is known in advance, and in the other case we must estimate the lag. Having to estimate the lag complicates matters, but we will include one possible technique in Sec. V.

Third, we assume that the time series is stationary, which implies that the mean, variance, and covariances at the various lags do not change over time. Therefore, if, for example, $f(x)=$ $a_{1} x$, we require $\left|a_{1}\right|<1$. Note that we have chosen a linear function $f$ to give an example of a restriction imposed by the stationarity requirement. In general, we simply require $f$ to admit a stationary distribution for the time series. It can be difficult to demonstrate that a particular nonlinear $f$ and particular distributions for the error term $\varepsilon_{t}$ admit a stationary distribution. Because our intent is estimation, we simply assume that the given time series is stationary or can be made stationary by differencing.

Almost all of the recent nonlinear time series literature has treated observational, but not system noise, and is concerned with estimating some property of $f$ in Eq. (3-2). The most ambitious goal would be to estimate $f$ itself, which we do not attempt. Rather, in Secs. IV and $V$ we collect several new tools for estimating certain features of $f$ (Lyapunov exponents, correlation dimension, conditional mean, and conditional variance). Note that if $f$ were known, then any of these features would be known. 


\section{TRADITIONAL METHODS}

In this section we focus on a particular sequence known as an MB sequence. However, the proposed methods can be tried on other time series that arise in nuclear materials control, such as shipper-receiver differences, operator-inspector differences, or measurement control data.

Traditionally, the analysis of data from nuclear materials control and accounting involves the idea of a material balance period, which includes shipments and receipts of material for processing. At the close of a material balance period, inventory is measured and a material balance might be

$$
M B=I_{b}-I_{e}+S-R,
$$

where $M B$ is the material balance, $I_{b}$ and $I_{e}$ are beginning and ending inventories, $S$ is shipments, and $R$ is receipts. A typical example might consist of monthly calculation of the material balance for one year. This results in a time series of 12 serially correlated observations. All measurements are made with error, including the inventory measurements. Because the beginning inventory for balance period $t$ is the ending inventory for balance period $t-1$, observations at time $t$ and time $t+1$ each depend on the inventory at time $t$ and are therefore correlated. For that reason alone, the MA(1) model is perhaps one reasonable model for the sequence $X_{1}$, $X_{2}, \ldots, X_{n}$, where $X_{n}$ is the $i$ th observed MB, if all balance periods have approximately the same throughput and ending inventory.

Correlation may also exist because of "systematic error." Systematic error can arise, for example, when measurements of multiple items are made with the same measurement method during the same calibration period. All measurements made during a single calibration period with the same instrument could be considered to be in one class. An intra-class correlation arises because the calibration error affects all measurements in the same direction, which helps explain the term systematic error. If there is systematic error, then a more complicated model than the MA(1) model is needed. Technical difficulties arise in applying either linear or nonlinear time series models if we cannot assume a fixed lag. The lag is not fixed, if, for example, an item remains in inventory for the first ten balance periods and then is removed from inventory. Conceivably, the lag could then change from nine to one. In ARMA models, the standard stationarity assumption requires that the lag does not change along the length of the sequence. . $^{3-1}$ The nonlinear analyses, to be presented in Secs. IV and V, can be modified to accommodate a changing lag, but those modifications will not be discussed in this report. Therefore, for the present report we assume that at any time $t$ along the series, the same number of previous observations have an effect on $X_{t}$. If the calibration frequency for a measuring instrument 
varied, say from one month to three months, then the lag would change over time. Therefore, we assume a fixed recalibration schedule is in effect for the measurement process. If so, then an MA(q) model is a reasonable linear model for the resulting MB sequence.

The main issue in the analysis of an MB sequence is whether apparent loss, which MB measures, is a true loss. It is commonly assumed that measurement errors of the fundamental measurements that comprise inventories, shipments, and receipts are well estimated. Repeated application of the delta method to the fundamental measurement error variances is used to estimate the variance of the MB statistic. ${ }^{3-6}$ The nuclear safeguards literature commonly refers to the application of the delta method as variance propagation. The idea behind variance propagation is to estimate the variance of a function of a random variable from an estimate of the variance of the random variable itself. For example, if the random variable has variance $\sigma_{X}^{2}$ and mean $\mu_{X}$, then it is usually a good approximation to assume that the random variable $Y=1 / X$ has variance $\sigma_{Y}^{2} \approx \sigma_{X}^{2} / \mu_{X}^{4}$. It is common in safeguards work to require an estimate of the ratio of two random variables because of physical relationships such as the relations between mass, density, and volume. Estimates of variance may be substituted for the actual variance as needed. For further details see Ref. 3-6 and the references therein.

An appeal to the central limit theorem (each term in the material balance equation is usually composed of a large number of measurements) is then usually sufficient justification to assume that the sequence $\left\{X_{1}, X_{2}, \ldots X_{t}\right\}$ is distributed approximately as $\operatorname{MVN}(\mu, \Sigma)$. Recall that we are using $X_{i}$ to denote the $i$ th observed MB, which is a random variable because of measurement error. Note that under this paradigm, we need not assume that the lag is fixed because the covariance matrix $\Sigma$ can be any positive definite $t$-by- $t$ covariance matrix. That is, we can allow $X_{4}$ to be correlated with both $X_{3}$ and $X_{2}$, while $X_{10}$ might only be correlated with $X_{9}$. The lag is therefore equal to two for $X_{4}$ but equal to one for $X_{10}$. This is an advantage of the present methods in use for analyzing MB sequences, although it does require the tedious estimation of each covariance in the variance-covariance matrix.

Under the assumption that $\left\{X_{1}, X_{2}, \ldots, X_{t}\right\}$ is distributed approximately as $\operatorname{MVN}(\mu, \Sigma)$, there are several ways to test $\mathbf{H}_{0}: \mu=0$. It is common to invoke a sequential method, such as Page's test so that a statistical test can be performed at the end of each balance period.

For an introduction to sequential analysis of MBs, see Refs. 3-7 and 3-8. Several studies (see Ref. 3-9 for example) have considered which statistical tests perform best under which loss scenarios ( $\mu$ defines the loss scenario). The multivariate normal assumption, which is used in all of these earlier studies, implies that the expected $M B_{t}$ is a linear function of some subset of the previous MBs. The break with tradition is to drop the linearity assumption. 


\section{DATA ANALYSIS}

Several estimation techniques have recently appeared in the nonlinear time series literature. To illustrate a few techniques and to test their usefulness for safeguards, we have applied five graphical techniques and one analytic technique on an MB sequence of 144 balance periods (12 years of monthly data, see Ref. 3-10) and on simulated data sets, including several simulated data sets that have properties similar to the MB data.

We have used the $S+$ computer package for graphics and to generate some of the simulated data. We have written FORTRAN programs to generate some of the simulated data and to perform some of the calculations needed to generate statistics that are used in the graphical and analytical tests.

The following is a brief description of the graphical techniques, four of which require the lengthy calculations that are implemented in the FORTRAN programs.

1) The first graphical technique is the pairwise scatterplot of $x_{t+1}$ versus $x_{t}$. As mentioned in Sec. $I$, this simple plot can be very revealing and should always be examined.

2) The second graphical technique applies nonparametric estimation of conditional means, $\mathrm{E}\left(X_{t+1} \mid X_{t}=x\right)$, and conditional variances, $\mathrm{E}\left(X_{t+1}^{2} \mid X_{t}=x\right)-\left\{\mathrm{E}\left(X_{t+1} \mid X_{t}=x\right)\right\}^{2} . \mathrm{A}$ nonparametric estimate of the lag $=1$ conditional mean is

$$
\hat{M}(x)=\left[\frac{1}{n-1} \sum_{j=1}^{n-1} X_{j+1} k\left(\frac{x-X_{j}}{h}\right)\right] /\left[\frac{1}{n} \sum_{j=1}^{n} k\left(\frac{x-X_{j}}{h}\right)\right],
$$

where $k$ is called the kernel. It is usually assumed that $k(x)$ evaluated at $x=0$ is the maximum value of $k$ and that $k(x)$ is a decreasing function of $|x|$. It is usually further assumed that $k$ is a symmetric probability density function such as the standard normal density. The parameter $h$ is the bandwidth, which determines the amount of smoothing. For more detail, see Refs. 3-3 and 3-4, but the idea in Eq. (3-11) is straightforward. We have $n$ observations, $X_{1}, X_{2}, \ldots X_{n}$ and seek an estimate of $X_{t+1}$ given the value $X_{t}=x$. The idea is to use all of the first $n-1$ observations, but weigh most heavily the observations that are nearest the value $x$. Suppose that $X_{10}$ $\approx x$. Then we would heavily weigh the value of $X_{11}$ in the estimate of the conditional mean of $X_{t+1}$ given the value $X_{t}=x$. By use of a kernel $k(x)$, which has its maximum value at $x=0$, Eq. (3-11) weighs most heavily those observations $X_{t+1}$ that have $X_{t}$ close in value to $x$.

Loosely speaking, if the bandwidth $h$ is very large, then relative to $h$, all observations are close in value to $x$, which is regarded as heavy smoothing.

Note that we are considering the lag $=1$ case, but a nonparametric estimate of the conditional mean and variance is also available if the lag $d>1$. 
A plot of $\mathrm{E}\left(X_{t+1} \mid X_{t}=x\right)$ versus $x$ is simple to interpret. It is linear if the true model is linear (and if we have the correct lag, $d=1$ ). Let $\hat{M}$ and $\hat{V}$ denote nonparametric estimates of the conditional mean and conditional variance. There are some technical details that make the choice of smoothing parameter $h$ used by the statistics $\hat{M}$ and $\hat{V}$ difficult. The main difficulty is analogous to the well-known difficulty in choosing the smoothing parameter in nonparametric density estimation. To see this, consider that the simplest nonparametric density estimator is the histogram. The histogram's appearance depends strongly on the choice of bin width. A wide bin contains all the data and we have oversmoothed. Narrow bins show spurious detail.

It remains a technical problem to find good $\hat{M}$ and $\hat{V}$. For simulated data sets for which the true conditional means and variances were known, long time series, of length 500 or more, are probably needed. Assuming the estimators $\hat{M}$ and $\hat{V}$ perform well, simple plots of $\hat{M}$ versus $x_{t}$ and $\hat{V}$ versus $x_{t}$ can reveal nonlinearity.

We show an example in Fig. 3-4. Figure 3-4a is a scatterplot of the 144 observations of the $\mathrm{MB}$ sequence mentioned above, in the form $x_{t+1}$ versus $x_{i}$. If we view the scatterplot as one would for simple regression, we would fit the best line through the points. The best (in the sense of least squares) line is shown. If we view the scatterplot as one would for nonparametric estimation of the conditional mean, we would fit a curve through the points. One such curve is shown. In Fig. 3-4 we used the S+ function supsmu(), which is a scatterplot smoother. Our FORTRAN program, which implements the nonparametric conditional mean estimation, gives very similar results. Figure $3-4 b$ is the same scatterplot as the one in Fig. $3-4 a$, but with the four largest observations trimmed. We suspected that the four large observations were influencing the supsmu() function and that if they were trimmed then the nonparametric estimate would be more nearly linear for the MB sequence. We trimmed them by replacing each of the four largest observations with a normal random variable having a mean equal to the estimated conditional expectation of the observation and having a variance equal to the sample variance of the sequence. Our purpose is not to discuss how to treat outlying observations but to choose a reasonable way to trim the outliers so that they do not have undue influence on the estimate of the conditional mean. It does appear that for the central portion of the data, a linear function is adequate.

We show another example in Figs. 3-5, 3-6, and 3-7, using the $\operatorname{map} X_{t}=\bmod \left(4 \varepsilon_{t-1}, 1\right)$ $+\varepsilon_{t}$, where $\varepsilon_{t} \sim N(0,1)$. Figure 3-5 is a histogram and normal probability plot of 1000 observations from the map. A normal probability plot is a standard plot to test normality by plotting the ordered observations versus the expected values of the order statistics from a normal distribution. It appears that the marginal distribution of the map is reasonably well approximated by a normal distribution. Because of the $\bmod ($ ) function, the map is nonlinear, and it is difficult to calculate the true marginal distribution of this map. 


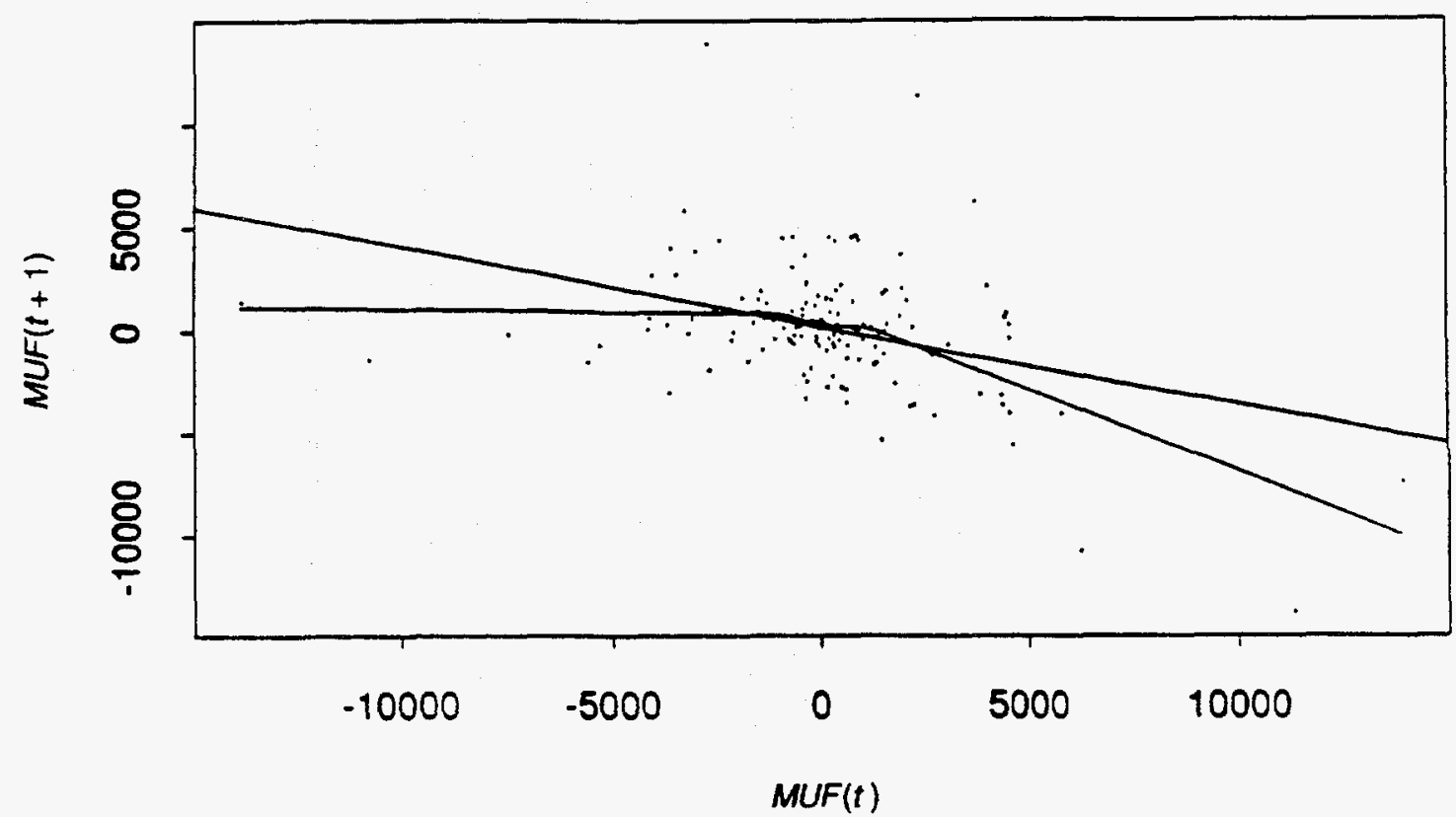

(a)

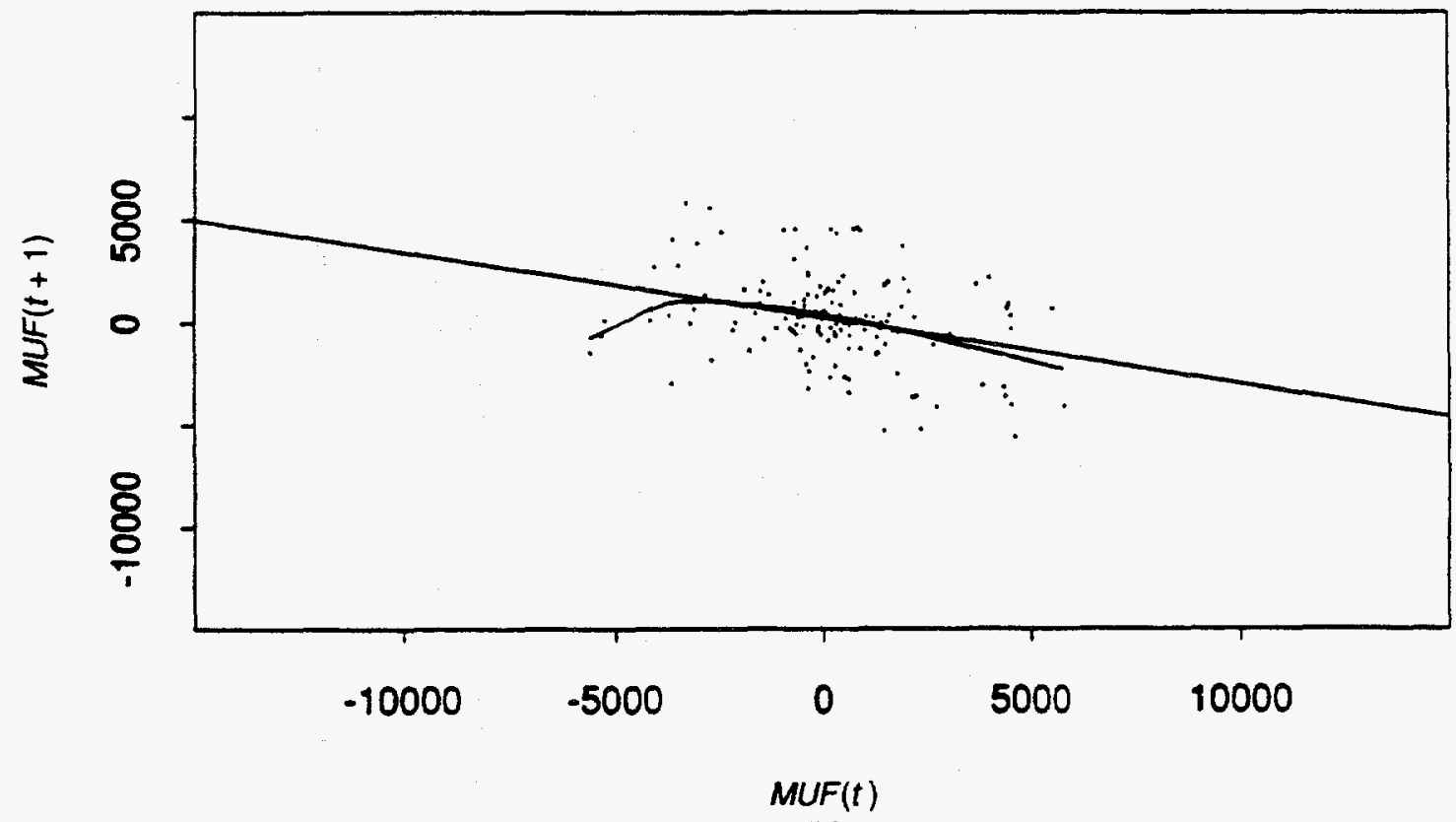

(b)

Fig. 3-4. Analysis of MUF data: (a) $M U F(T+1)$ vs. $M U F(t)$;

(b) $M U F(t+1)$ vs. $M U F(t)$, outliers trimmed. 

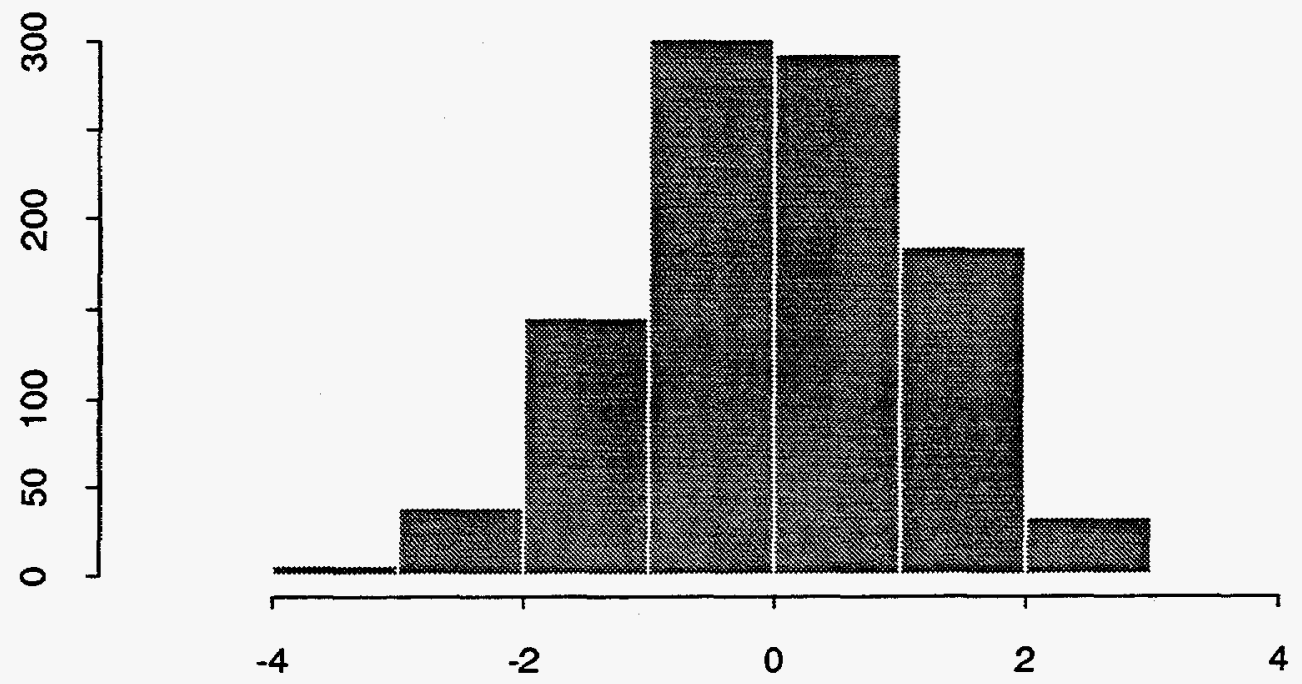

Histogram of observations

(a)

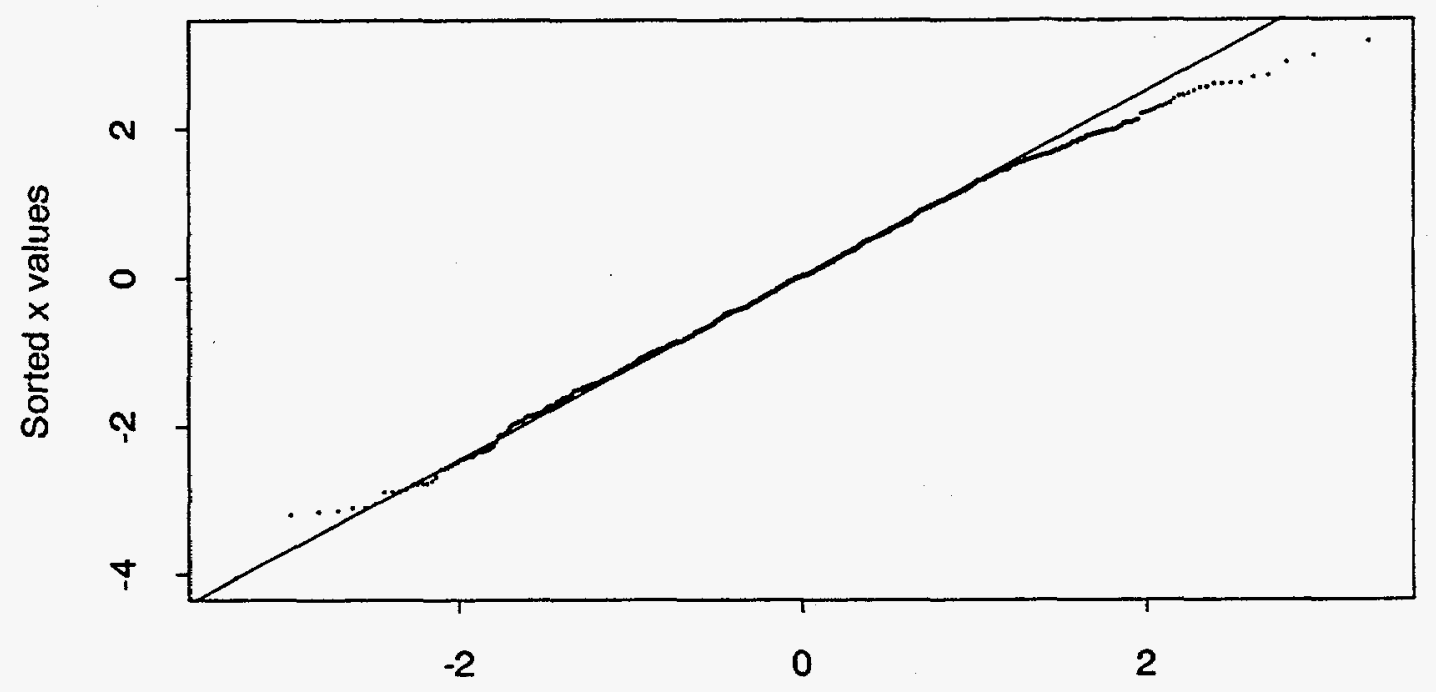

Quantiles of standard normal

(b)

Fig. 3-5. Histogram and normal probability plot of 1000 observations from the map $X_{t}=\bmod \left(4 \varepsilon_{t-1}, 1\right)+\varepsilon_{t}$, where $\varepsilon_{t} \sim N(0,1)$. 


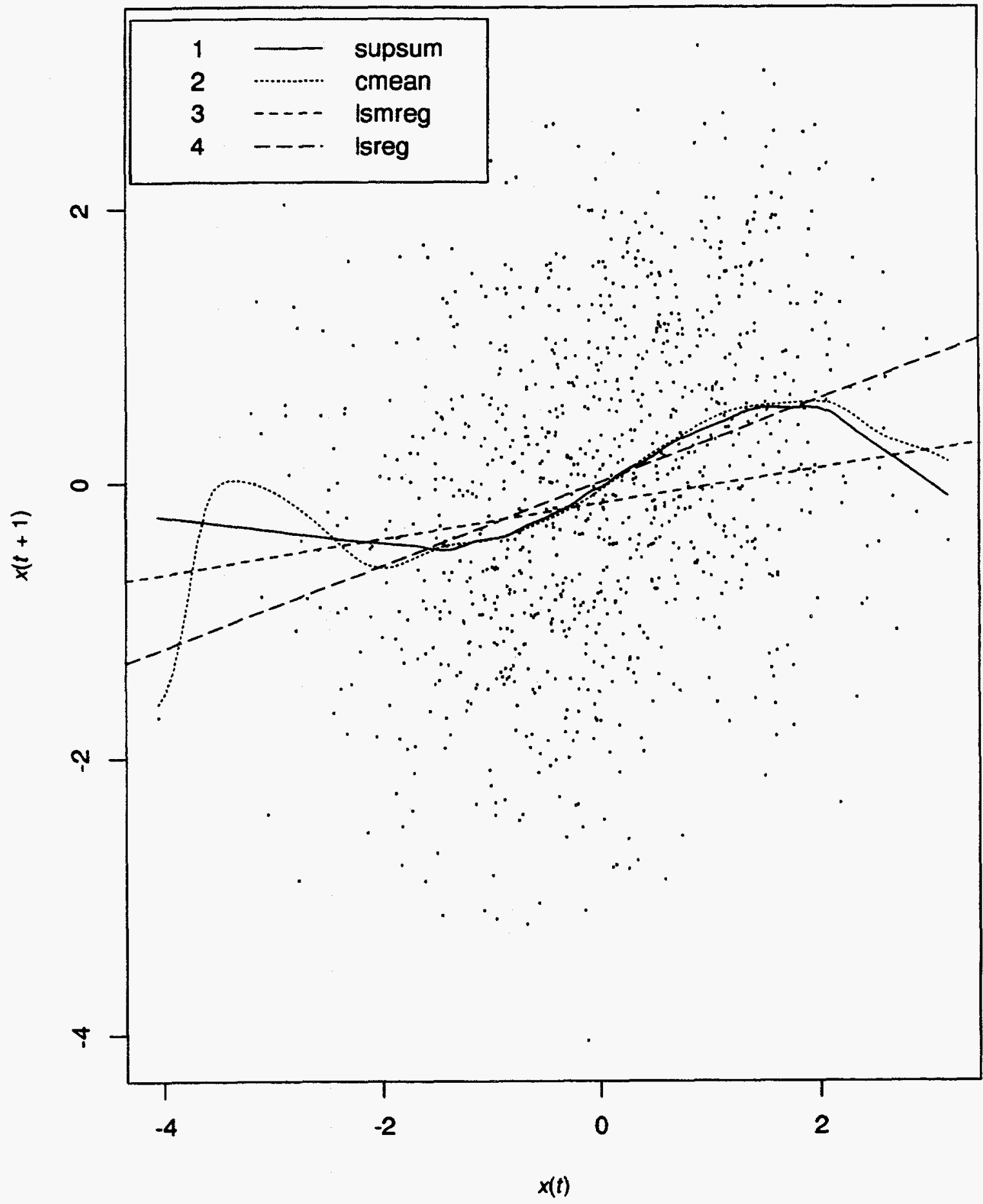

Fig. 3-6. Four ways to model the dependence of $x_{t}$ on $x_{t-1}$. 


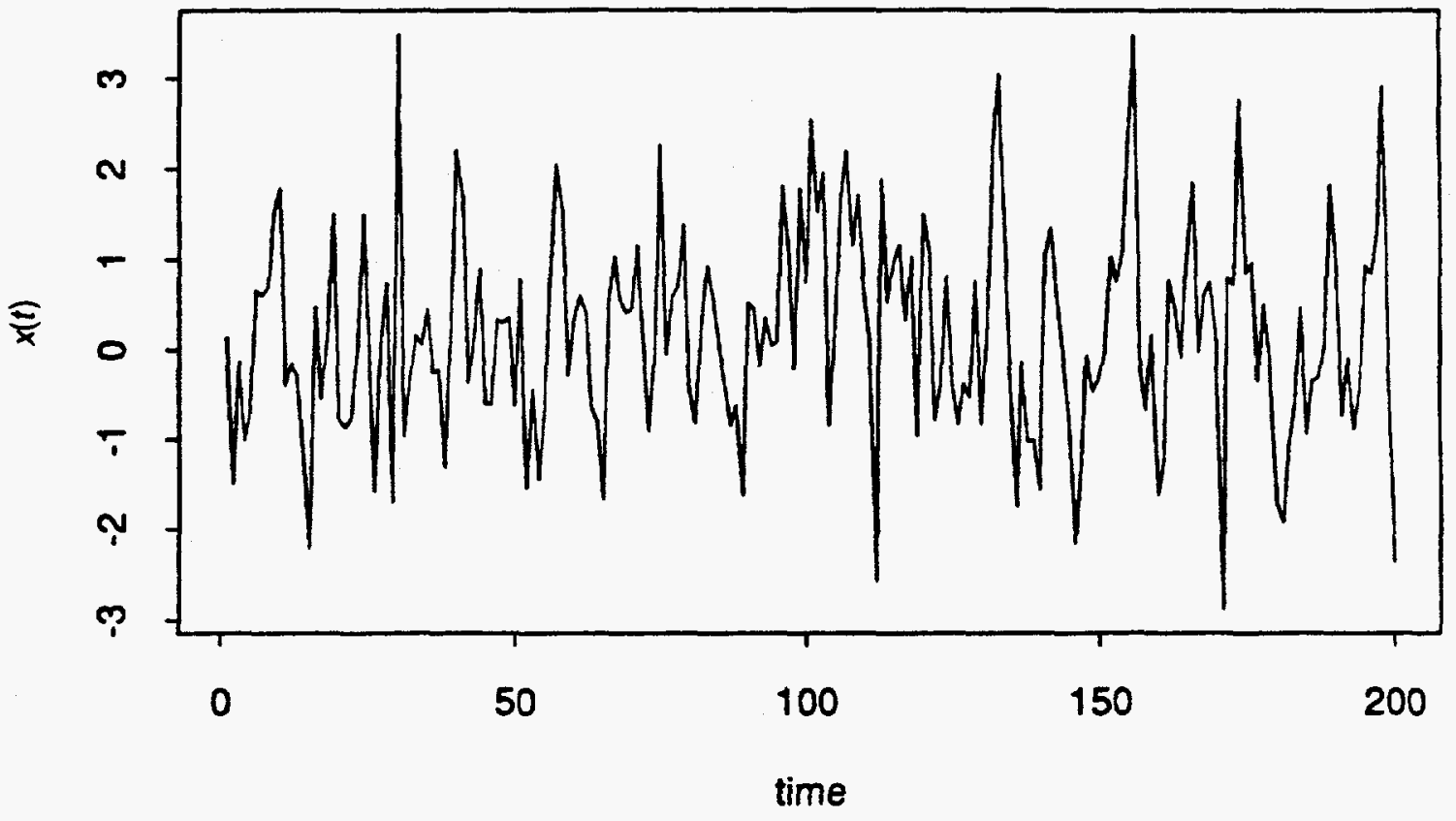

Series : $\mathbf{x}$

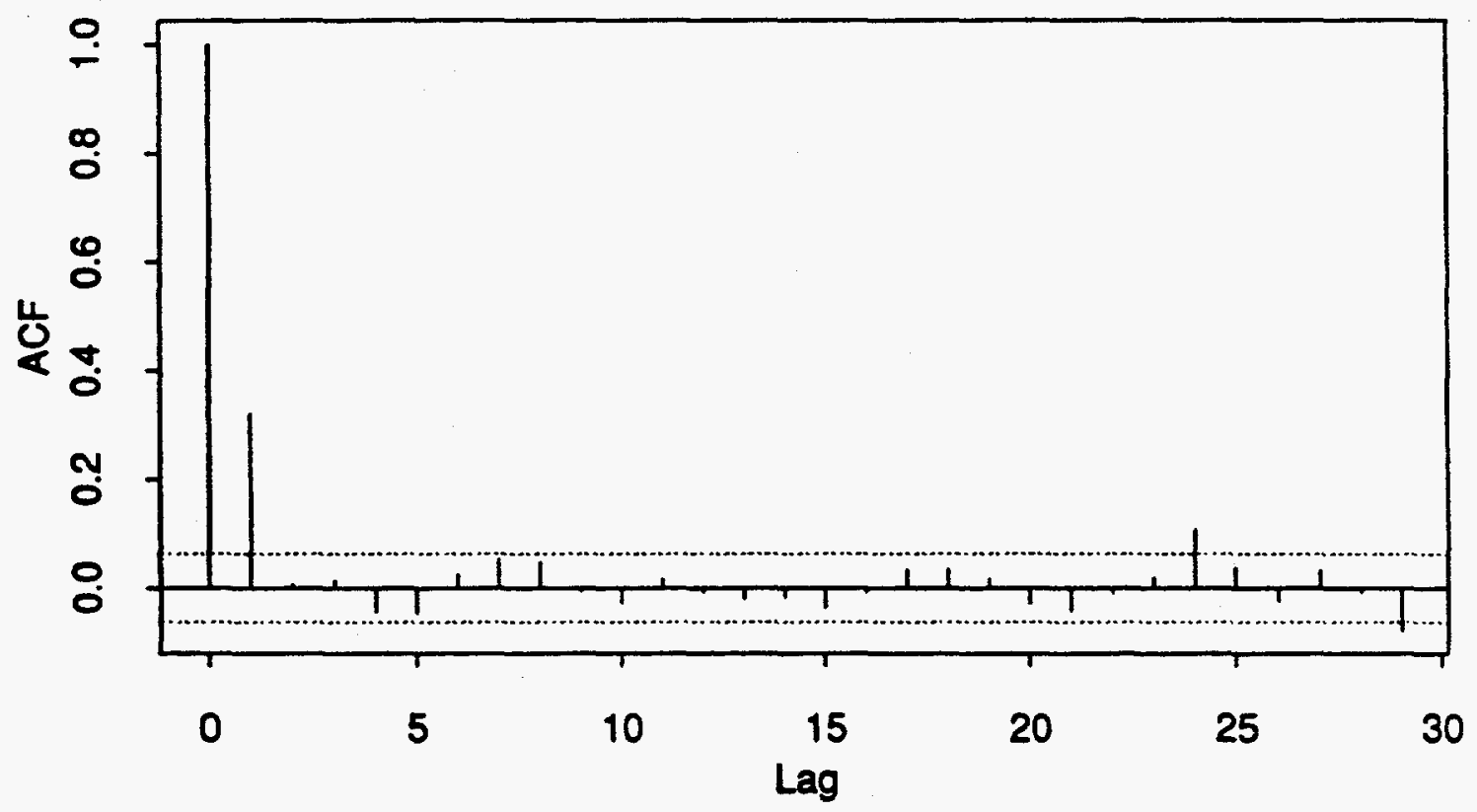

Fig. 3-7. The time series $X_{t}=\bmod \left(4 \varepsilon_{t-1}, 1\right)+\varepsilon_{t}$, where $\varepsilon_{t} \sim N(0,1)$. 
In Fig. 3-6 we illustrate four ways to model the dependence of $x_{t}$ on $x_{t-1}$. The curve labeled supsmu is the result of a scatterplot smoother in $S+$. The curve labeled cmean is the result of the conditional mean estimate from one of our FORTRAN programs. The two lines are the linear fits to the scatterplot for the least median squares and the least sum of squares. The departure from linearity is not very strong in Fig. 3-6. In Fig. 3-7, we show a time series plot of the first 200 observations from the map and the autocorrelation function, which suggests that an MA(1) model might be reasonable if we did not know what generated the data.

In Fig. 3-8, we show the result of applying our conditional mean estimator to an MA(1) map. We used 1000 observations from an MA(1) model and predicted the next 100 observations, which are shown in Fig. 3-8. We have performed several similar experiments with linear maps to compare the performance of linear and nonlinear methods. It appears that little is lost by modeling a linear map with a nonlinear model, provided that the number of observations is about 200 or more.

Using the map $X_{t}=\left(\varepsilon_{t-1}^{2}-1\right)+\varepsilon_{t}$, where $\varepsilon_{l} \sim N(0,1)$, we show another example in Figs. 3-9, 3-10, and 3-11. Figure 3-9 is a histogram and normal probability plot of 1000 observations from the map. The departure from normality is obvious. In Fig. 3-10 we show the result of the supsmu() function applied to the plot of $X_{t}$ versus $X_{t-1}$. The nonlinear dependence of $X_{t}$ on $X_{t-1}$ is apparent. However, either the MA or AR models fitted to this map show essentially no linear dependence. The result of a linear estimation is compared to a nonlinear estimation in Fig. 3-11. Note that the linear estimator is essentially constant, so that the prediction of each observation using linear estimation is simply the sample mean.

In Fig. 3-12, we show a time series plot and compare linear to nonlinear estimation on a scatterplot smoother of observations from the map $X_{t}=-\sin \left(x_{t-1}+\varepsilon_{t-1}\right)+\varepsilon_{t}$. Again, the slope of the line from a linear fit is small because the true conditional mean is an increasing function over part of the range and a decreasing function over other parts of the range. We return to this example in Sec. VI.

The estimate $\hat{M}$ can also be used for an analytic approach: namely, to compare linear with nonlinear models for prediction. In Sec. VI we compare the prediction capabilities of linear and nonlinear estimation schemes, with a view toward sensitive detection of material loss.

If the conditional mean and variance were well estimated and turned out to be nonlinear, then one might look for physical explanations that lead to better understanding of the physical processes, including measuring instrument performance, that generate the data. 


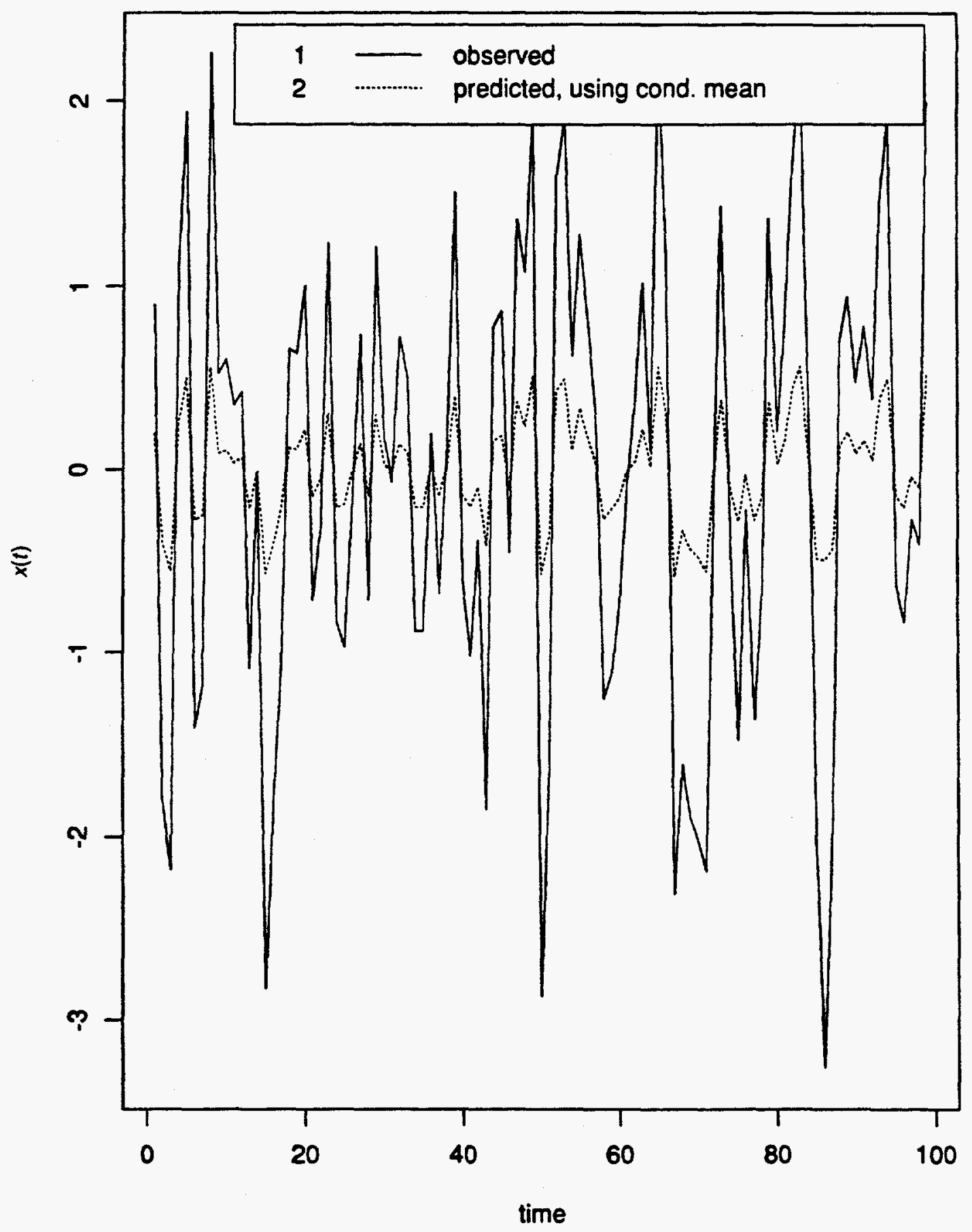

Fig. 3-8. Performance of conditional mean estimate. 


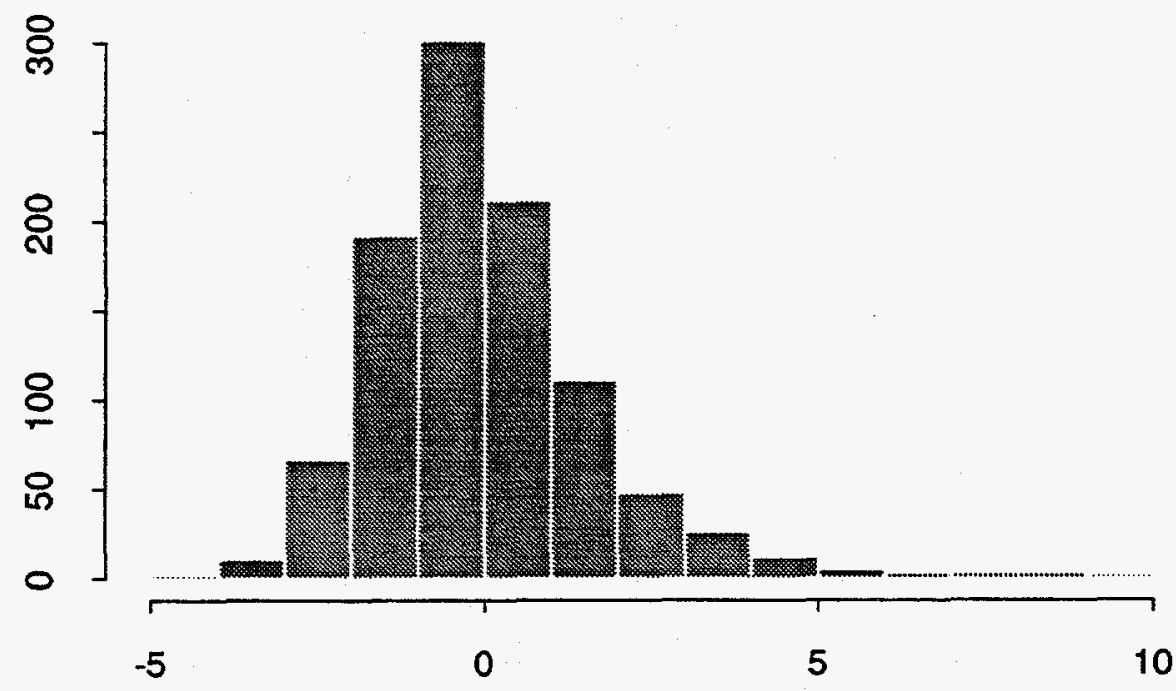

Histogram of observations

(a)

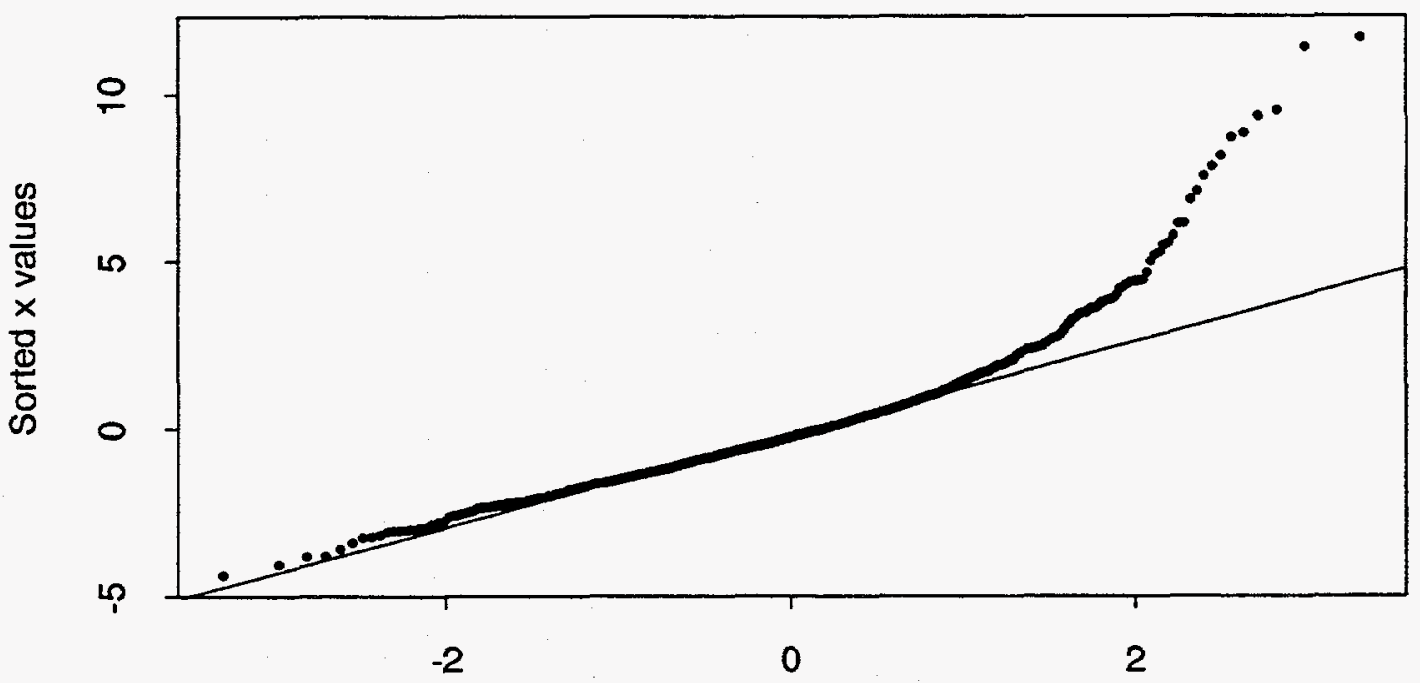

Quantiles of standard normal

(b)

Fig. 3-9. Histogram and normal probability plot of 1000 observations from the map $x_{t}=\left(\varepsilon_{t}^{2}-1\right)+\varepsilon_{t}$, where $\varepsilon_{t} \sim N(0,1)$. 


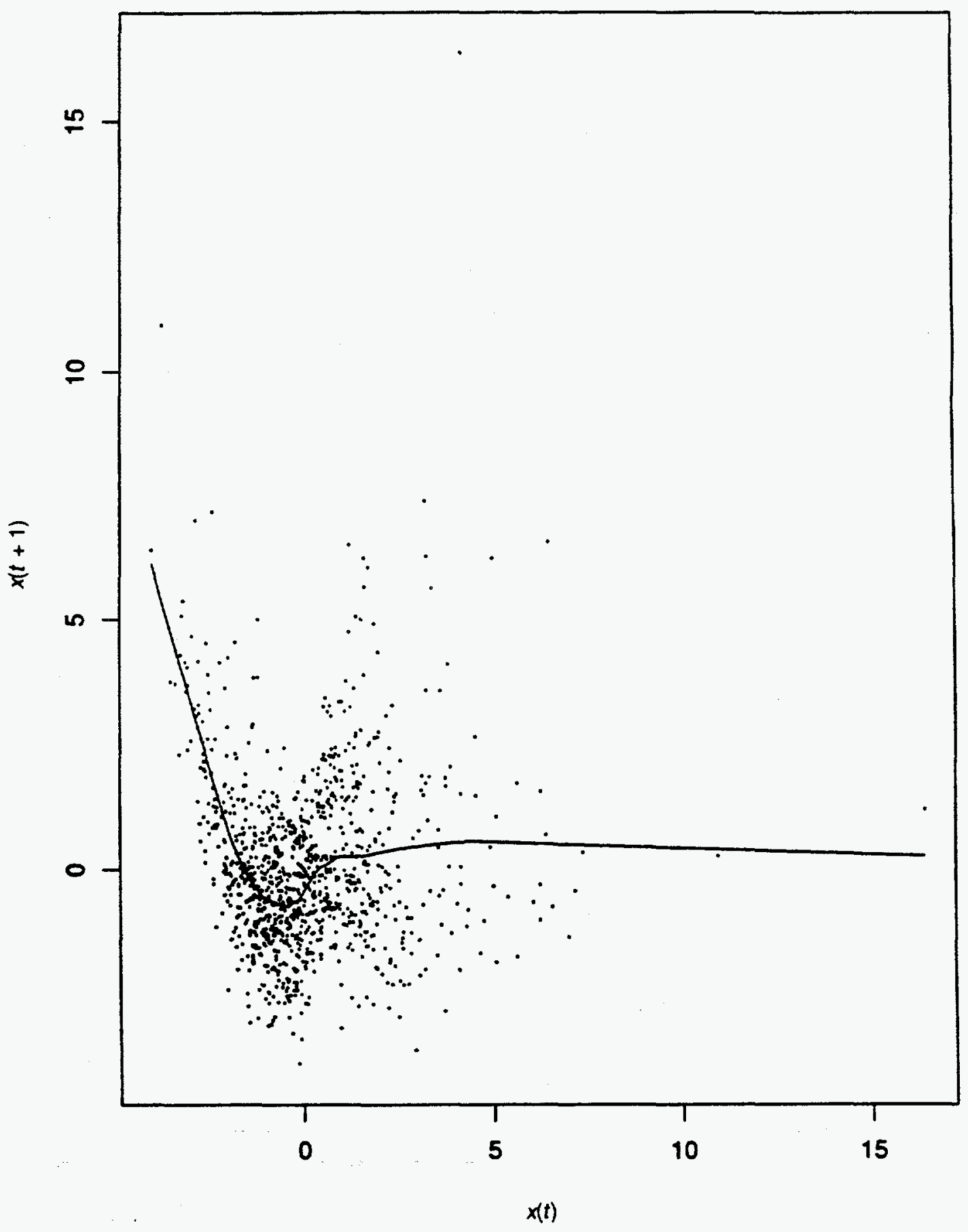

Fig. 3-10. Result of supsmu() function applied to plot of $X_{t}=\left(\varepsilon_{t}^{2}-1\right)+\varepsilon_{t}$, where $\varepsilon_{t} \sim N(0,1)$. 


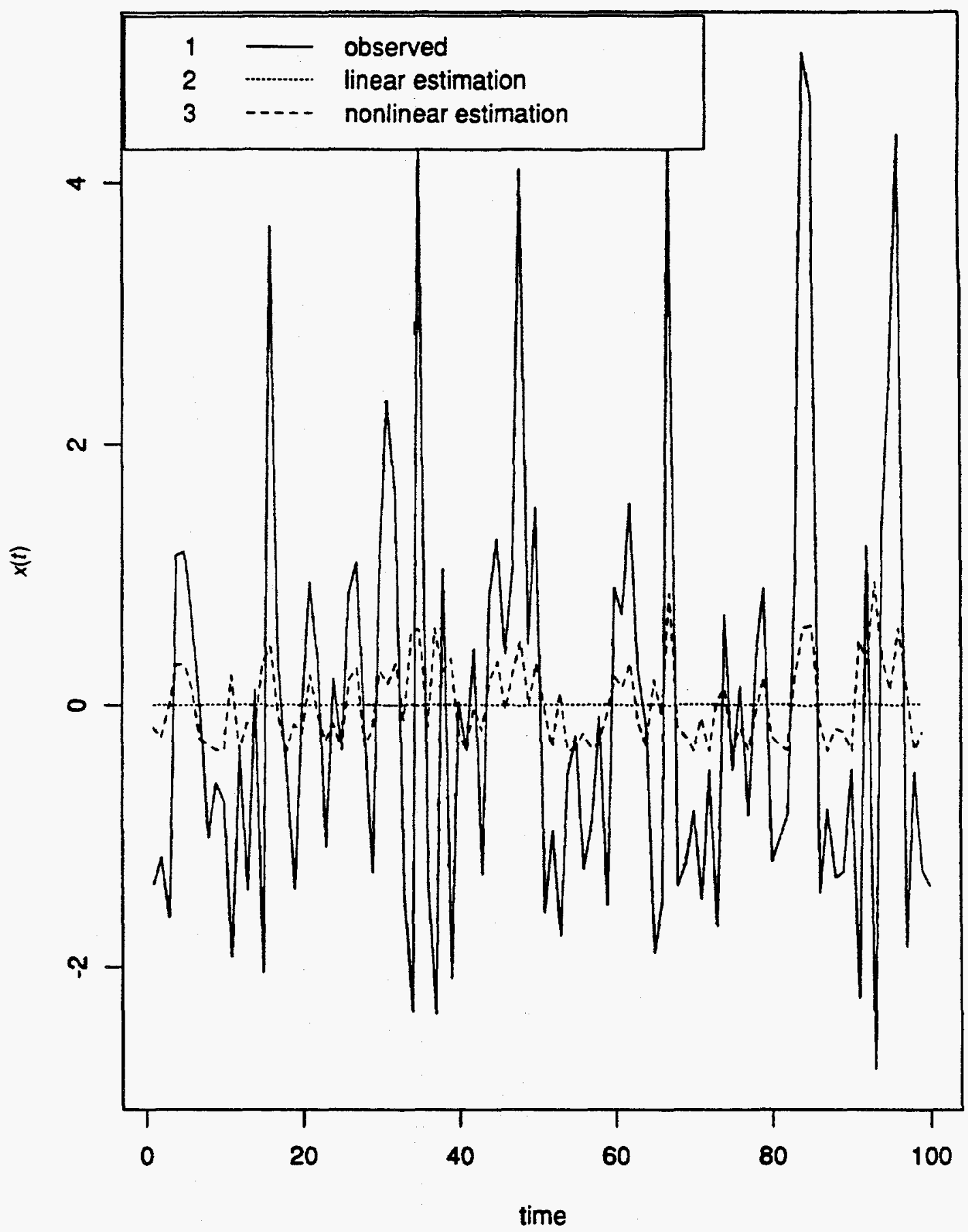

Fig. 3-11. Comparing linear to nonlinear estimation for $X_{t}=\left(\varepsilon_{t}^{2}-1\right)+\varepsilon_{t}$, where $\varepsilon_{t} \sim N(0,1)$. 


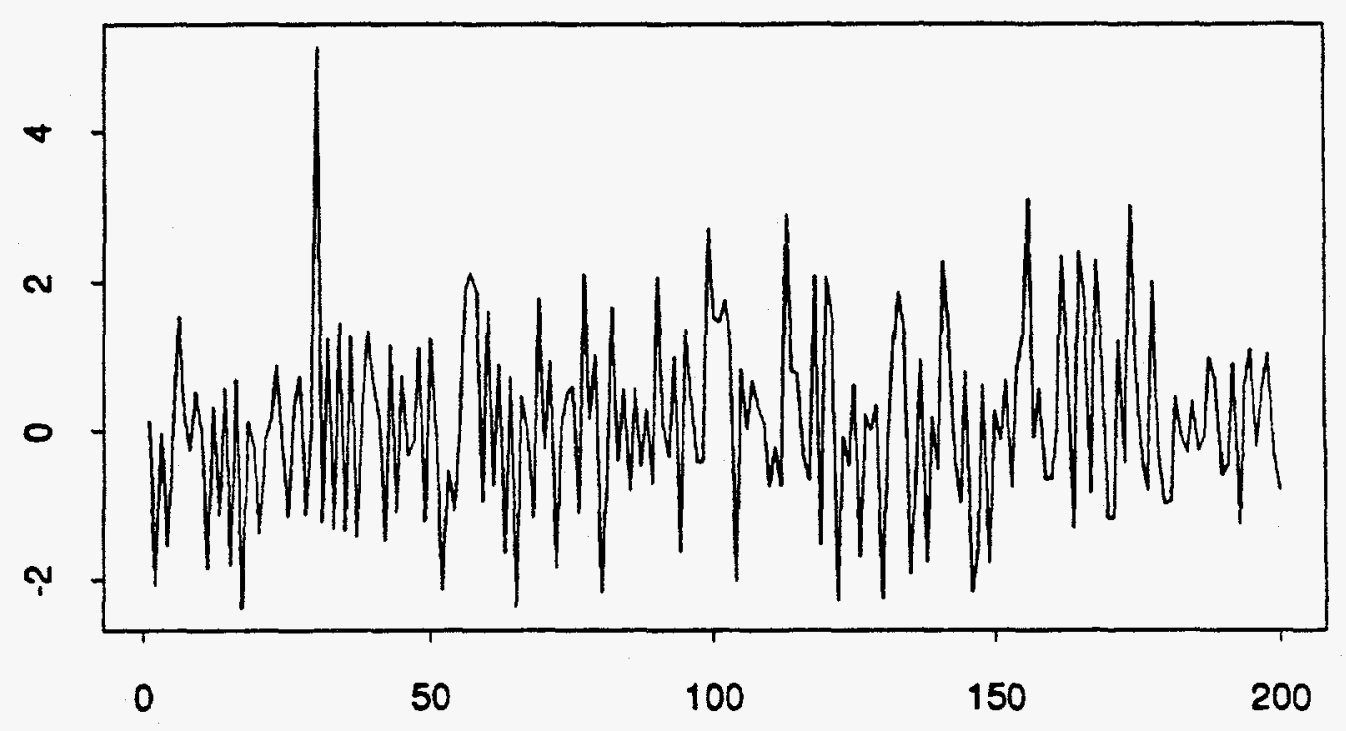

Fig. 3-12a. Times series plot.

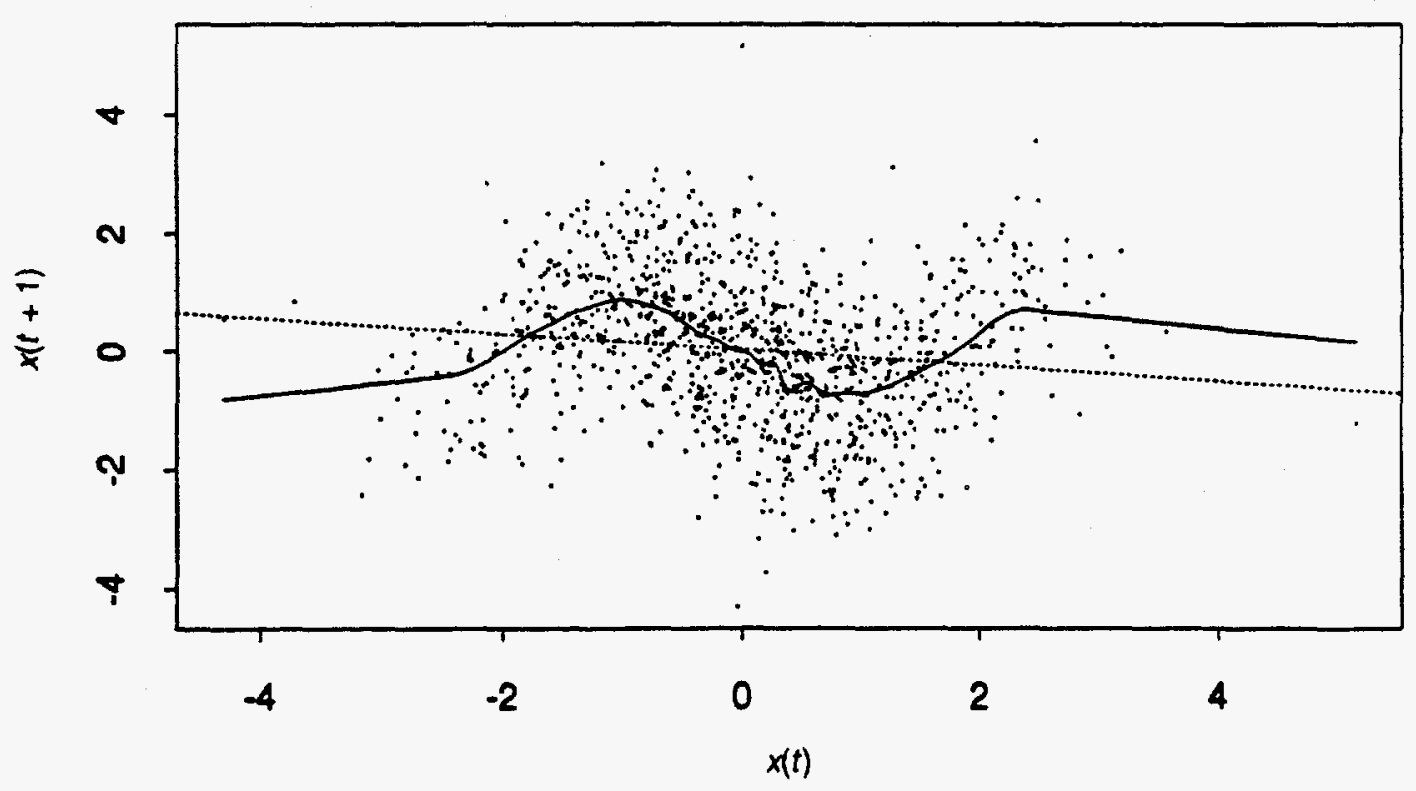

Fig. 3-12b. Nonlinear vs. linear estimation. 
3) The third graphical technique to detect nonlinearity is to graph the cumulative sum of the estimates $\hat{M}$, given in Eq. (3-11) above, versus $x$. The graph should be quadratic if the true model is linear. There are two advantages $3-11$ of this graph over the graph of $\hat{M}$ versus $x$. First, the cumulative sum of the estimates $\hat{M}$ is less sensitive to the bandwidth. Second, approximate confidence bands (analogous to confidence intervals but applying throughout the range of $x$ ) can be derived based on results in Ref. 3-11. In Figs. 3-13, 3-14, and 3-15 we apply this technique to the AR(1) map, the logistic map observed without error, and the logistic map observed with error, respectively. In these three examples it is clear from the plots of the conditional mean itself whether the map is linear, but the examples serve to illustrate the method.

4) The fourth graphical technique plots estimates of local Lyapunov exponents (LLEs) versus the observed sequence. ${ }^{3-12}$ The idea is that for small lags, the LLEs for a nonlinear time series will depend on the value of the time series. A suitable definition of the LLE is

$$
\lambda_{i, m}=\frac{1}{m} \frac{1}{n_{i}} \sum_{j \in S_{i}} \log \left|\left(X_{i+m}-X_{j+m}\right) /\left(X_{i}-X_{j}\right)\right|
$$

for $i=1,2, \ldots, n-m$, where $S_{i}=\left\{j: 0<\left|X_{i}-X_{j}\right| \leq \mathrm{h}\right\}, n_{i}=$ number of elements in $S_{i}, m \in$ $\mathbf{N}$, and $h>0$. In Eq. (3-12), $\lambda_{i, m}$ is called the LLE at $X_{i}$ for lag $m$, and $h$ is the bandwidth.

Again, because there is a connection between nonparametric density estimation and the estimation of Lyapunov exponents or of LLEs, the most pressing technical difficulty is the choice of bandwidth $h$. That technical difficulty aside, this graphical technique seems promising. We have tested it on the logistic map observed without error and on a simple AR(1) model. The plot of the estimated LLEs at lag $m=1$ for the AR(1) map is shown in Fig. 3-16a, and the same plot is shown for the logistic map in Fig. 3-17a. Note that the plot for the AR(1) map shows only random variation around a horizontal line, whereas the plot for the logistic map is curved. This is truly a feature that should distinguish certain nonlinear maps $f$ from linear maps. It is probably most useful in higher dimensions where simpler graphical techniques are not available.

To see why the LLE, say for lag $m=1$ defined in Eq. (3-12), depends on $X_{i}$ for most nonlinear $f$, consider the logistic map given in Eq. (3-3). It is not difficult to see that the expected value of $X_{i+1}-X_{j+1}$ depends on the values $X_{i}$ and $X_{j}$ and not on simply $X_{i}-X_{j}$. In contrast, for example, in the AR(1) model, the expected value of $X_{i+1}-X_{j+1}$ depends on simply $X_{i}-X_{j}$. Therefore, the LLE for lag $m=1$ does not depend on $X_{i}$, and the plot of the estimated LLE should exhibit only random variation around a horizontal line. 


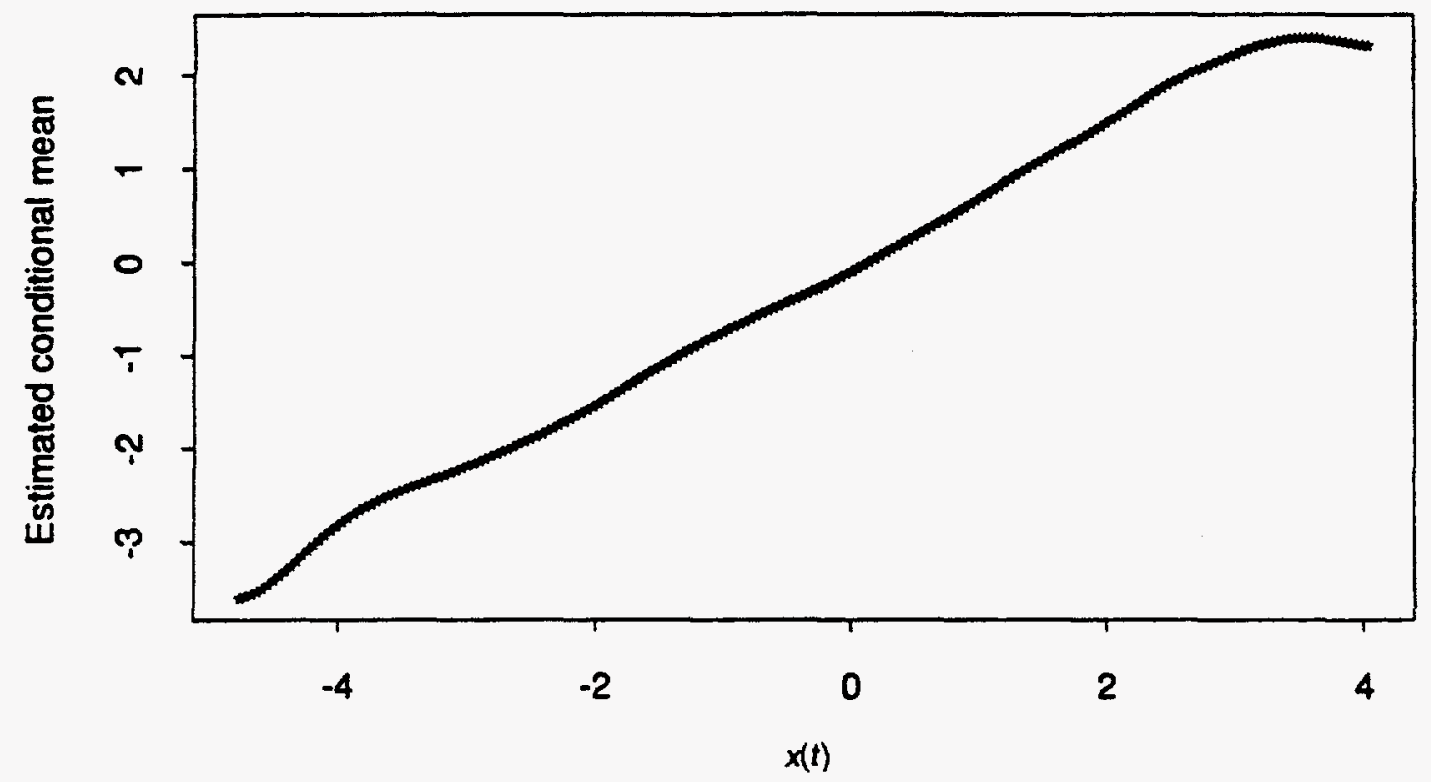

(a)

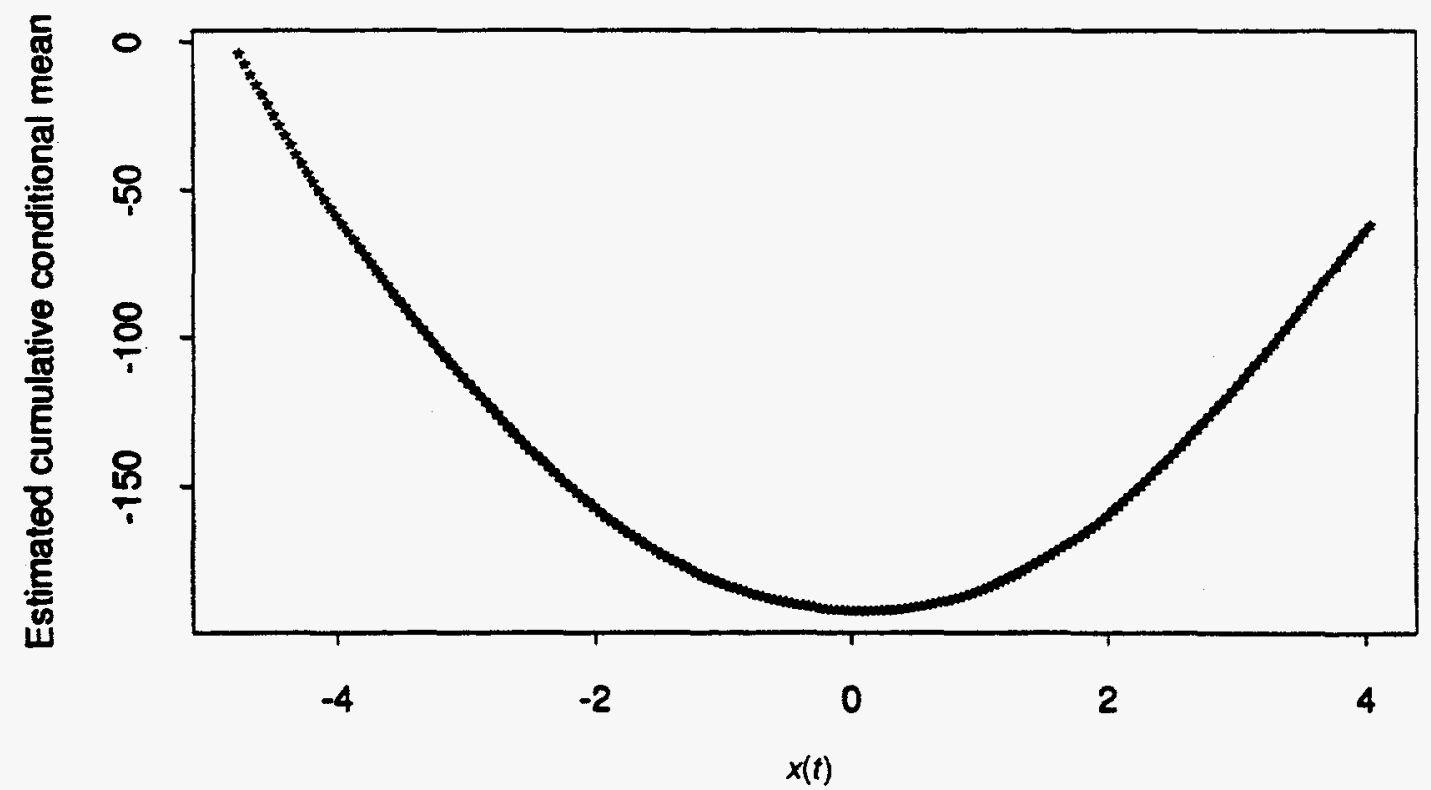

(b)

Fig. 3-13. AR(1) map. 


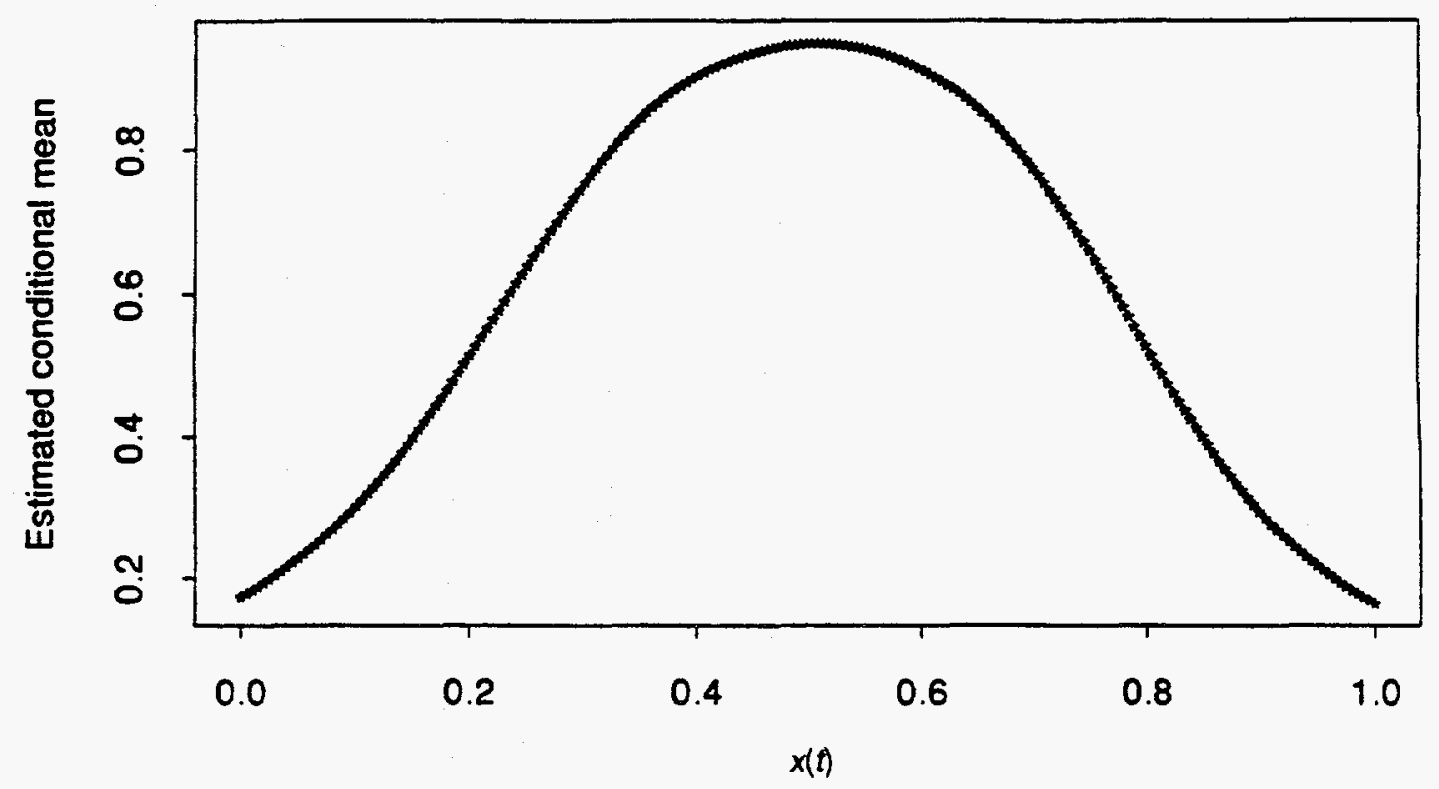

(a)

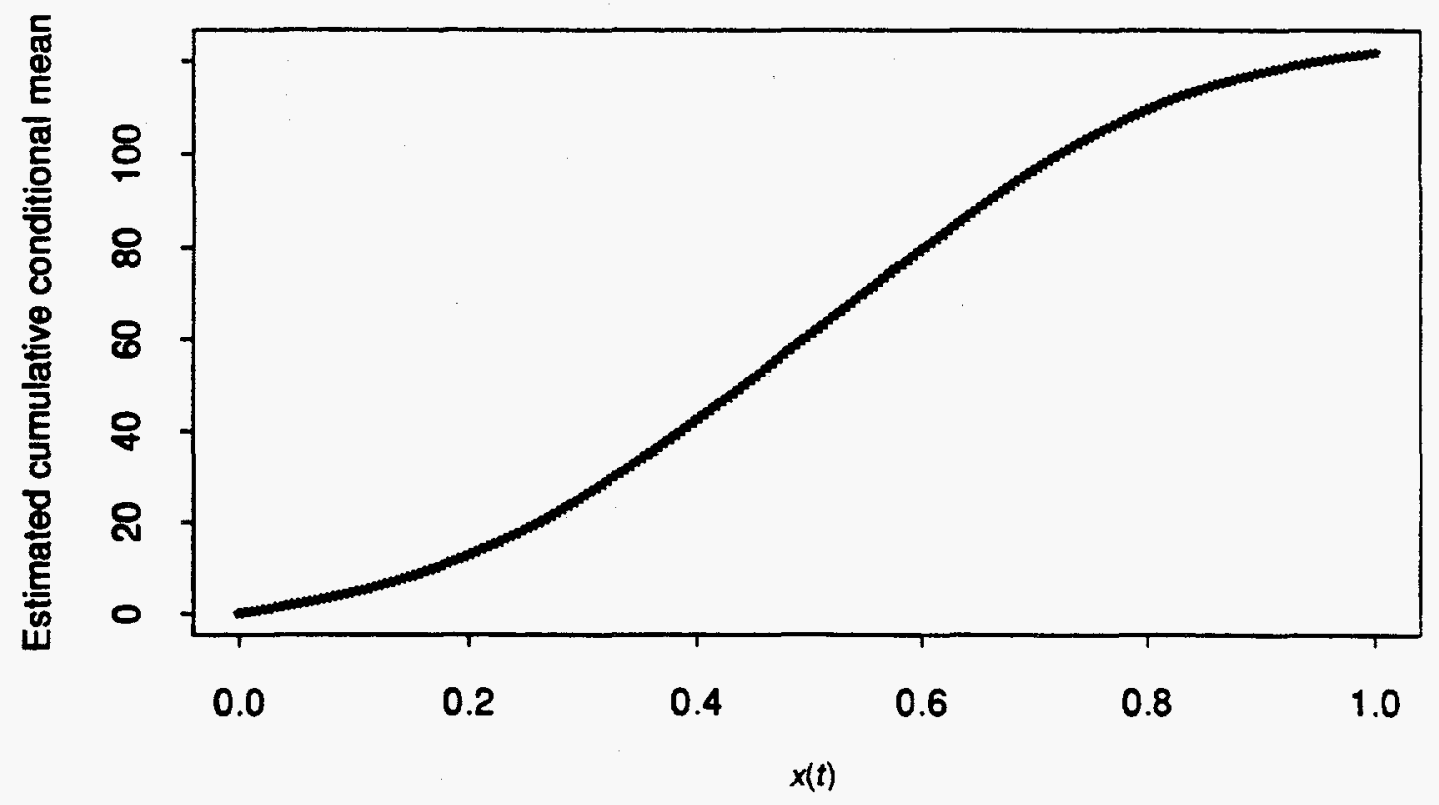

(b)

Fig. 3-14. Logistic map observed without error. 


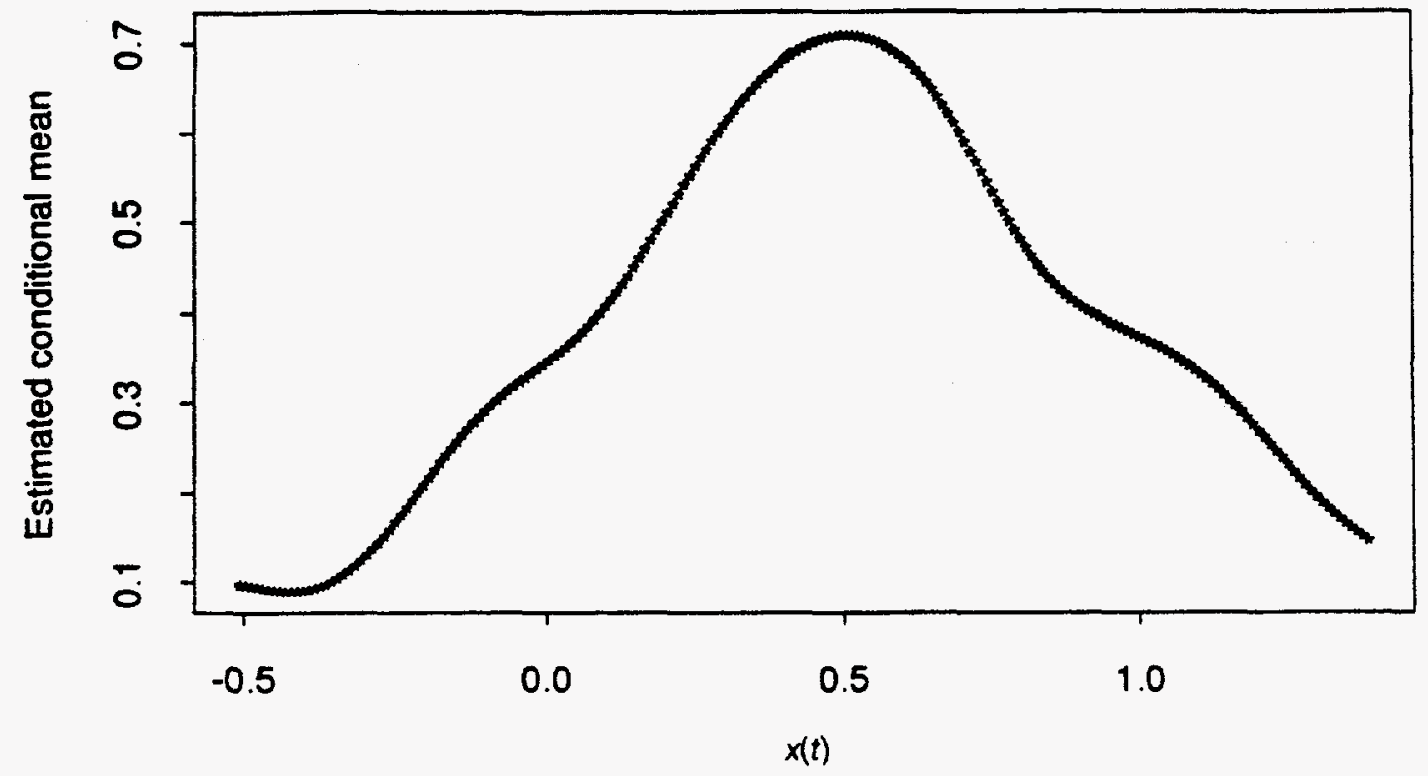

(a)

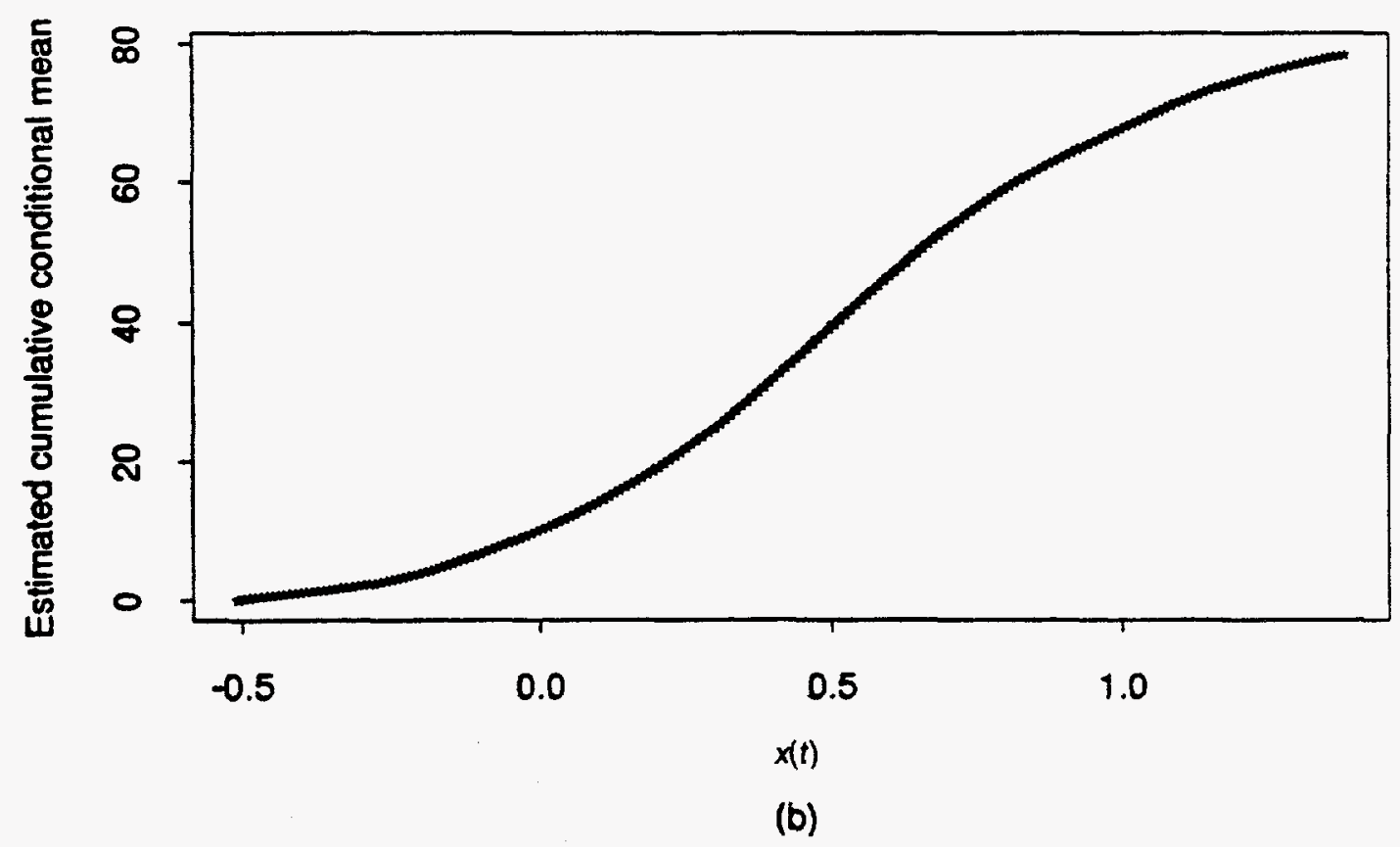

Fig. 3-15. Logistic map observed with error. 


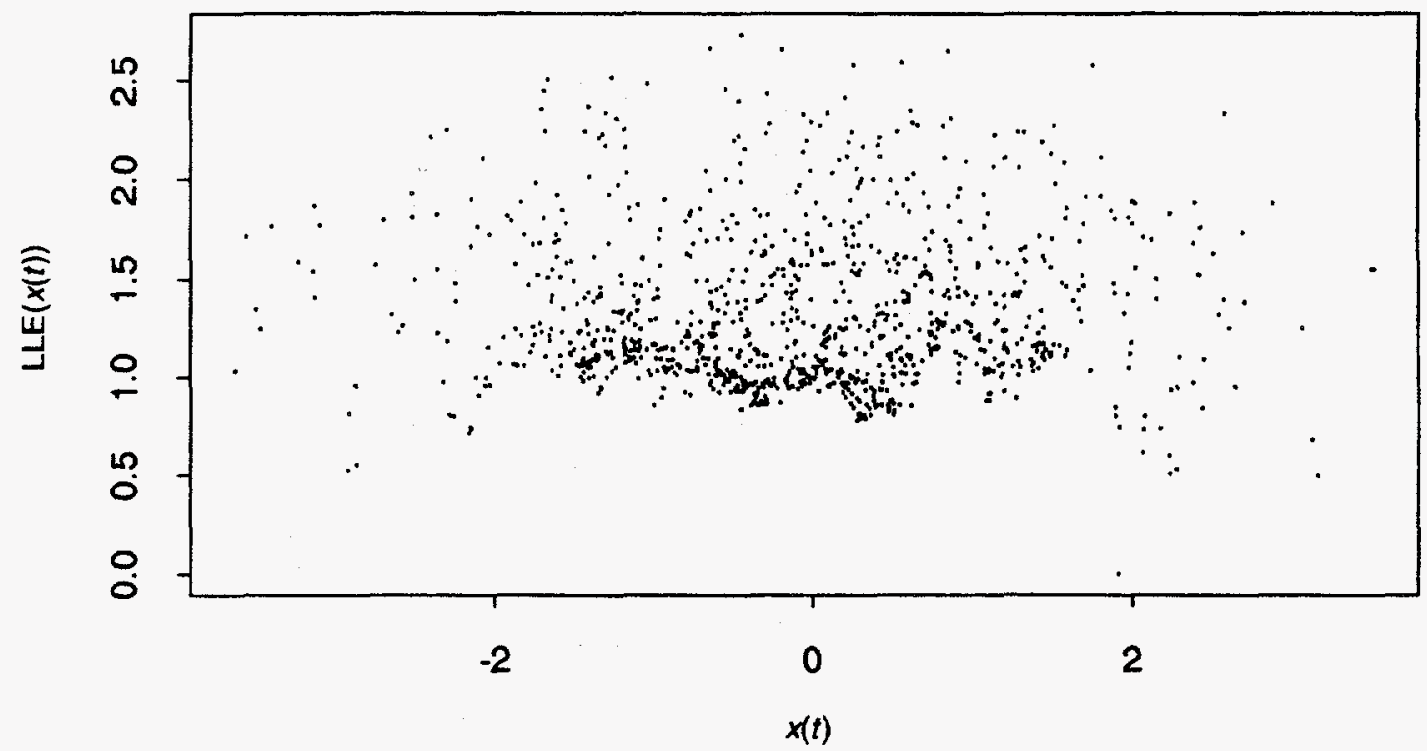

(a)

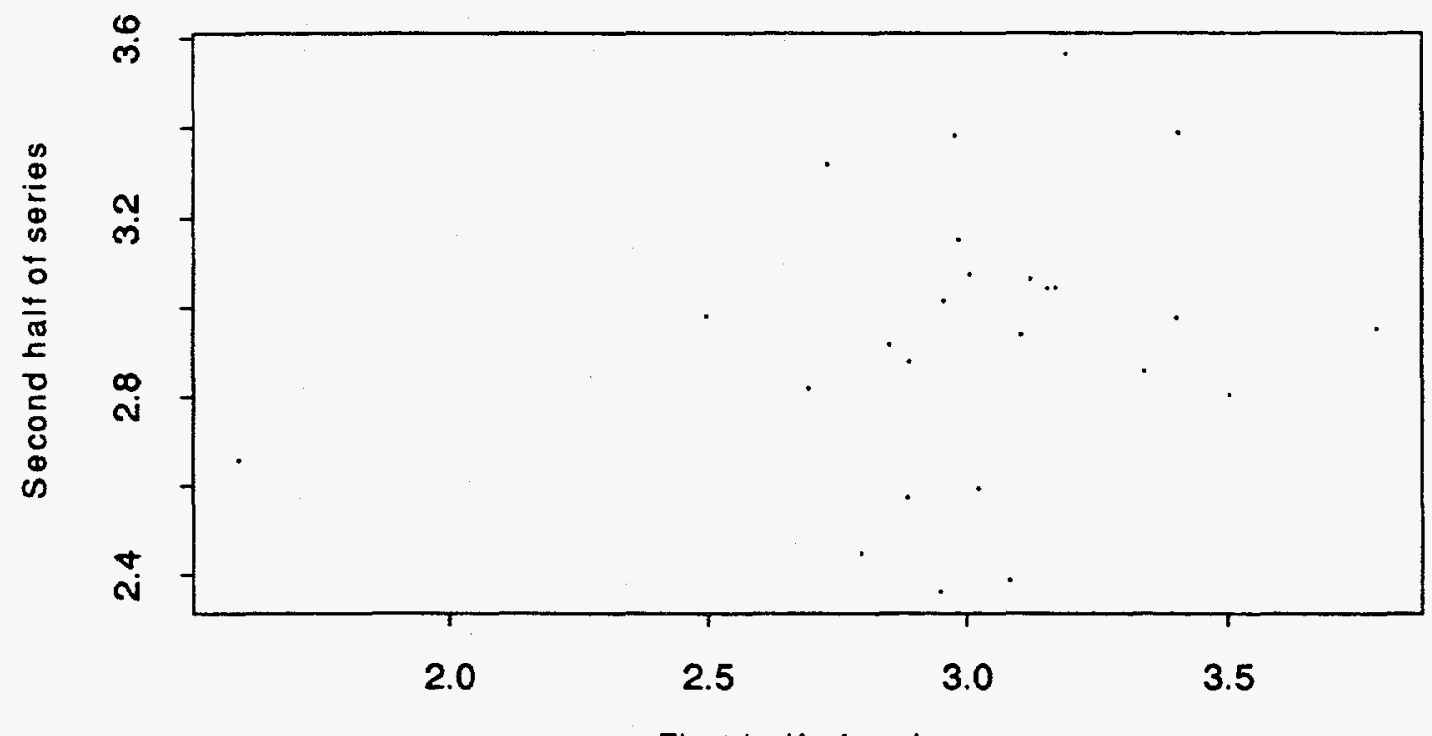

First half of series

(b)

Fig. 3-16. Local Lyapunov exponents for $A R(1)$ map. 


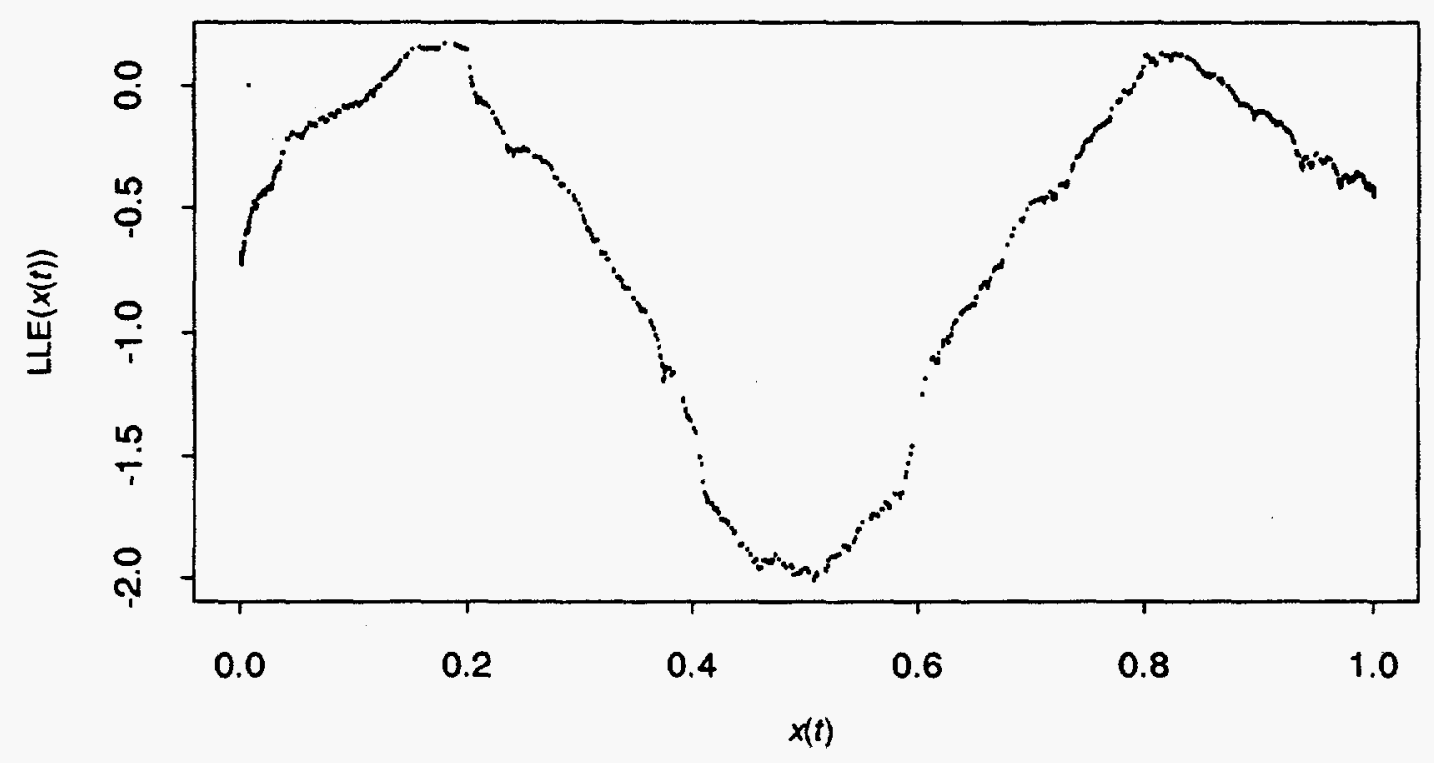

(a)

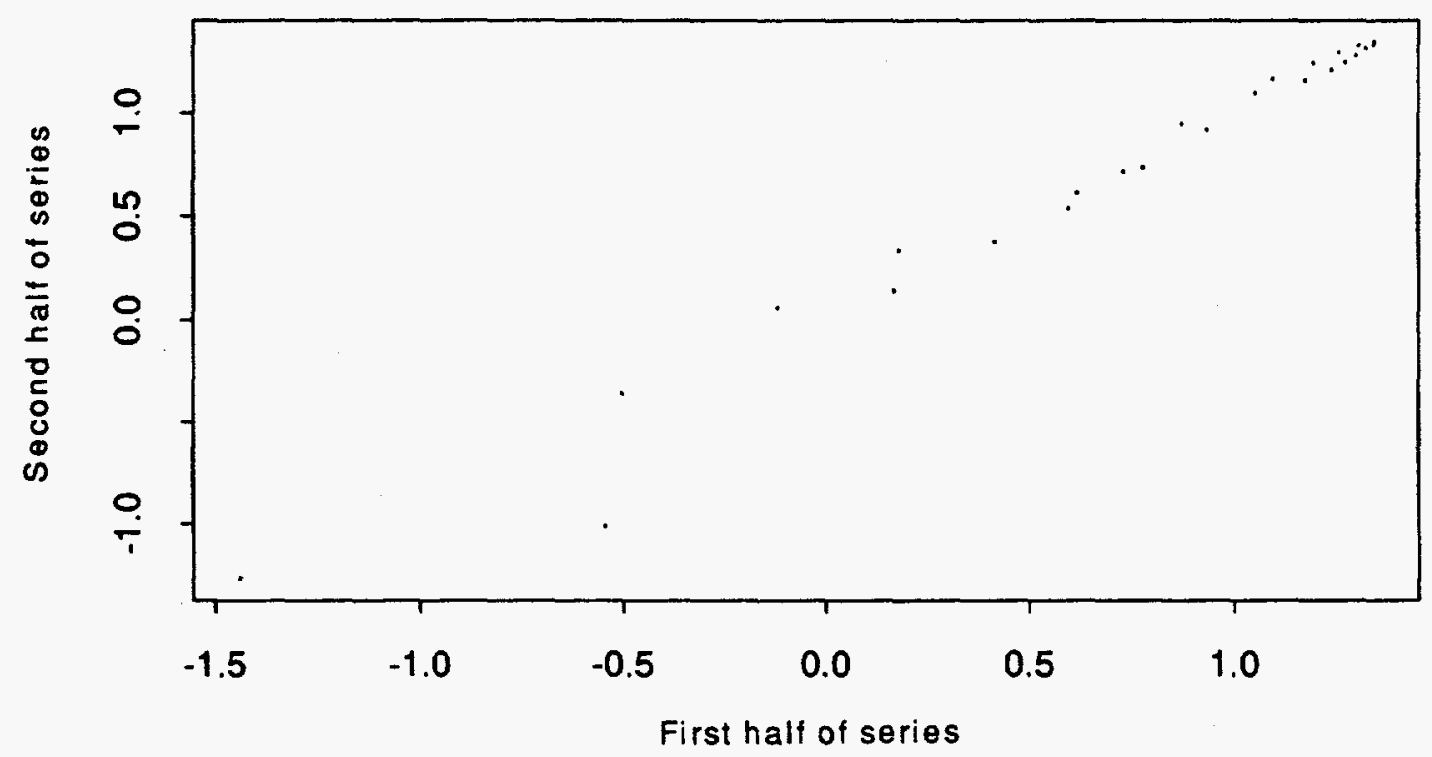

(b)

Fig. 3-17. Local Lyapunov exponents for logistic map, no error. 
5) The fifth graphical technique uses the LLEs as described in the fourth graphical technique. The time series is divided into a first and a second half. The range of the time series in the first half is partitioned into bins and the average LLE over all observations in a particular bin is recorded. This is done for each bin in the first half of the time series. The second half of the series is handled in the same way, and the average LLEs over each bin for the second half of the series are plotted versus those of the first half. The idea is that if the series is deterministic, the plot will be linear with unit slope. If the series is observed with error, there will still be a pattern in the plot for most (but not all) nonlinear maps $f$. We have just begun to experiment with this plot and do not have any conclusions yet.

We illustrate technique five in Figs. $3-16 \mathrm{~b}$ and $3-17 \mathrm{~b}$ for the AR(1) map and the logistic map, respectively. There are obvious patterns in Fig. 3-17b but not in Fig. 3-16b.

The analytical technique we have used is described in Chapter 5 (5.5, p. 225) of Ref. 3-13. This is a simple test with no specific alternative to linearity. On simulated data that was linear, the test performed correctly; that is, it failed to reject linearity. On the MB sequence where we currently do not suspect nonlinearity, linearity was not rejected. On data simulated from the logistic map with $\alpha=4$, the test rejected the hypothesis of linearity under several cases. In one case, the logistic map was observed (unrealistically) without noise, and in the other cases, observation errors were added with several different variances.

The idea behind several proposed tests for nonlinearity is to apply the Wold decomposition theorem.3-14 According to the theorem, the residuals from a linear model fitted to a time series are uncorrelated if the time series is linear. Nonlinearity can be detected by analyzing the residuals.

\section{ADDITIONAL ESTIMATION PROCEDURES}

In this section we list and describe a few other techniques that we have tested that have recently appeared in the literature.

\section{A. Estimating Dimension}

The goal is to estimate the dimension of an attractor in the presence of noise in the data. We detect chaos in a time series by deciding whether a sample of vector observations lies within a subset of possibly non-integer dimension.

Setting (without noise): Scalar time series $\left\{X_{n}\right\}$ satisfying

$$
x_{n}=f\left(x_{n-d}, x_{n-d-1}, \ldots, x_{n-1}\right)
$$


where $d$ is the embedding dimension and $f$ is nonlinear. As we have seen in Sec. III, for many $f$, the deterministic system does not converge to a fixed point or limit cycle but instead exhibits pseudo-random behavior known as chaos.

It can happen that, as $n \rightarrow \infty$, the $d$-vectors $\mathbf{y}_{n}=\left(x_{n-d+1}, \ldots, x_{n}\right)$ approach a limiting set known as the attractor. In a chaotic system, the attractor is typically a set with non-integral dimension; that is, a fractal, and in that case is also known as a strange attractor.

Associated with fractal attractors are numerous definitions of dimension, some can be estimated from numerical data. One of the methods used to detect chaos in time series is to fix the lag $d$, form the vectors $y_{n}=\left(x_{n-d+1}, \ldots, x_{n}\right)$, and estimate their fractal dimension. There is evidence of chaos if the estimated dimension settles down to a fixed number as $d$ increases. If the estimated dimension continues to grow with $d$, there is no evidence of a simple chaotic system, and a stochastic model is required. This procedure assumes that we observe the data on the attractor, so it is necessary for the system to quickly reach the attractor.

Among the many definitions of dimension, the easiest to estimate is the correlation dimension. Other definitions of dimension that appear in the nonlinear time series literature include Lyapunov, information, and pointwise dimension. For some maps, the dimensions can be calculated analytically (rather than estimated) and are not all the same. The correlation dimension has been the subject of the most statistical studies, primarily because it is the easiest to estimate, and is the only dimension discussed in this report. Reference 3-5 considers three cases: observing a sequence from Eq. (3-13) without noise, with observational noise, and with system noise. Observational and system noise were defined in Sec. III. Unfortunately, if the system is observed with error (as is always the case in practice) then even the correlation dimension is difficult to estimate. We now define the correlation dimension and give two methods of estimating correlation dimension. To allow the possibility of observational error, we use upper case letters to denote random quantities.

Define for the $n$ vectors $\mathbf{Y}_{1}, \ldots, \mathbf{Y}_{\mathrm{n}}$ in $\Re^{d}$

$$
C_{n}(r)=\frac{\sum_{i=2}^{n} \sum_{j=1}^{i-1} I\left(\left\|\mathbf{Y}_{i}-\mathbf{Y}_{j}\right\|\langle r)\right.}{n(n-1) / 2},
$$

where $I$ denotes the indicator function $I\left(\left\|\mathbf{Y}_{i}-\mathbf{Y}_{j}\right\|<\mathrm{r}\right)=1$ if $\left\|\mathbf{Y}_{i}-\mathbf{Y}_{j}\right\|<r$ and equals zero otherwise. Here, \|\|$\|$ is any norm, say the $L^{2}$ norm, in which case $\|\mathrm{x}\|=\left(x_{1}^{2}+x_{2}^{2}+\ldots+x_{d}^{2}\right)^{1 / 2}$ in $\Re^{d}$. Therefore, $C_{n}(r)$ is the fraction of all $\left(\begin{array}{l}n \\ 2\end{array}\right)$ pairs of vectors whose separation is less than r. $C_{n}(r)$ is the natural estimate of $C(r)=P\left(\left\|\mathrm{Y}_{i}-\mathrm{Y}_{j}\right\|<r\right)$. 
The limit

$$
C(r)=\lim _{n \rightarrow \infty}\left\{C_{n}(r)\right\}
$$

is called the correlation integral, and because for many systems $C(r)$ has a power law behavior for small $r[C(r)=a r p, r<\varepsilon$ for small $\varepsilon]$, the correlation dimension is defined as

$$
p=\lim _{r \rightarrow 0}\left\{\frac{\log C(r)}{\log r}\right\} \text {. }
$$

To be consistent with the notation in Ref. 3-5, we use $p$ to denote the correlation dimension. Recall that $p$ is also the usual notation for the lag in linear AR models. The meaning of $p$ should be clear from the context.

The most common procedure for estimating $p$ consists of plotting $-\log C_{n}(r)$ versus $-\log r$ and looking for a region where the plot is approximately linear. The slope over that region is the estimate of $p$. The lag $d$ is then increased until the estimates of $p$ appear to be stable.

One way to estimate $p$ from the plot of $-\log C_{n}(r)$ versus $-\log r$ is to use generalized least squares. Successive values of $\log C_{n}(r)$ are correlated and do not have equal variance, so generalized least squares is required.

Richard Smith ${ }^{3-5}$ proposes two estimators of $p$, which he claims "effectively achieves" generalized least squares by a different route.

Estimator 1 (The Binomial estimator):

Calculate $C_{n}(r)$ at $K+1$ points, $r=r_{0}>r_{1}>r_{k}$ for some integer $K$. For simplicity, assume $r_{j}=\varepsilon \phi^{j}$ for $0 \leq j \leq K$ with $\varepsilon>0$, and $0<\phi<1$. Also assume

$$
C(r)=a r p, r<\varepsilon
$$

Only consider those interpoint distances that are less than $\varepsilon$ for some small $\varepsilon$ and assume that these are the smallest order statistics from a sample of $n(n-1) / 2$ independent distances. This independent distance hypothesis (IDH) is valid in the limit as $\varepsilon \rightarrow \infty$. Let $N_{j}$ denote the number of interpoint distances less than $r_{j}$, so

$$
C_{n}\left(r_{j}\right)=\frac{N_{j}}{n(n-1) / 2}
$$


Under the $\mathrm{DHH}$, and using Eq. (3-17), the conditional distribution of $N_{j+1}$ given $N_{j}$ is $\operatorname{Bin}\left(N_{j}, \theta\right)$, where $\theta=C\left(r_{j+1}\right) / C\left(r_{j}\right)=\phi^{p}$. Therefore, the conditional joint distribution of $N_{1}$, $\ldots, N_{k}$, given $N_{0}$, is proportional to

$$
\prod_{j=0}^{K-1}\left(\phi^{p}\right)^{N_{j+1}}\left(1-\phi^{p}\right)^{N_{j}-N_{j+1}}
$$

This leads to the maximum likelihood estimator (MLE)

$$
\hat{p}=\frac{\log \left(\sum_{j=0}^{K-1} N_{j+1}\right)-\log \left(\sum_{j=0}^{K-1} N_{j}\right)}{\log \phi} .
$$

\section{Estimator 2:}

Fix $\varepsilon$, let $\phi \rightarrow 1$ and $\mathrm{K} \rightarrow \infty$. Consider the individual interpoint distances. Suppose there are $M$ individual interpoint distances less than $\varepsilon$, denoted $D_{1}, D_{2}, \ldots, D_{M}$. Under the $\mathrm{IDH}$ and assumption (3-17), the $D$ 's are conditionally independent with distribution function $\operatorname{Pr}\left(D_{m}<\right.$ $\left.r D_{m}<\varepsilon\right)=(r / \varepsilon)^{p}$, for $0<r<\varepsilon$. This leads to the MLE

$$
\hat{p}=\frac{M}{\sum_{m=1}^{M} \log \left(\varepsilon / D_{m}\right)},
$$

with approximate standard error $\hat{p} / \sqrt{M}$.

Reference 3-5 has more detail about estimating $p$ and how to modify $\hat{p}$ if Eq. (3-17) is a poor assumption or if the system is observed with error. In particular, if the assumption given in Eq. (3-17) needs to be modified, Ref. 3-5 suggests an alternative to the binomial estimator called the beta-binomial estimator. We have implemented the binomial and beta-binomial estimators as well as estimator 2 in a FORTRAN program. We illustrate some results on the Henon map $\left(x_{t}=1+0.3 x_{t-2}-1.4 x_{t-1}^{2}\right)$, which is a popular map in the nonlinear time series literature because of the non-integer (fractal) dimension of its attractor. We show 1000 observations from the map in Fig. 3-18a. From the unusual shape of this scatterplot, we might expect the map to have a non-integer dimension. However, note in Fig. 3-18b, where the map is observed with error, that the attractor looks less interesting. The error variance is only 0.04 in Fig. 3-18b, so very small errors can quickly spoil the interesting geometry of the attractor. Figure 3-19 shows dimension estimation results using the binomial estimator for the Henon 


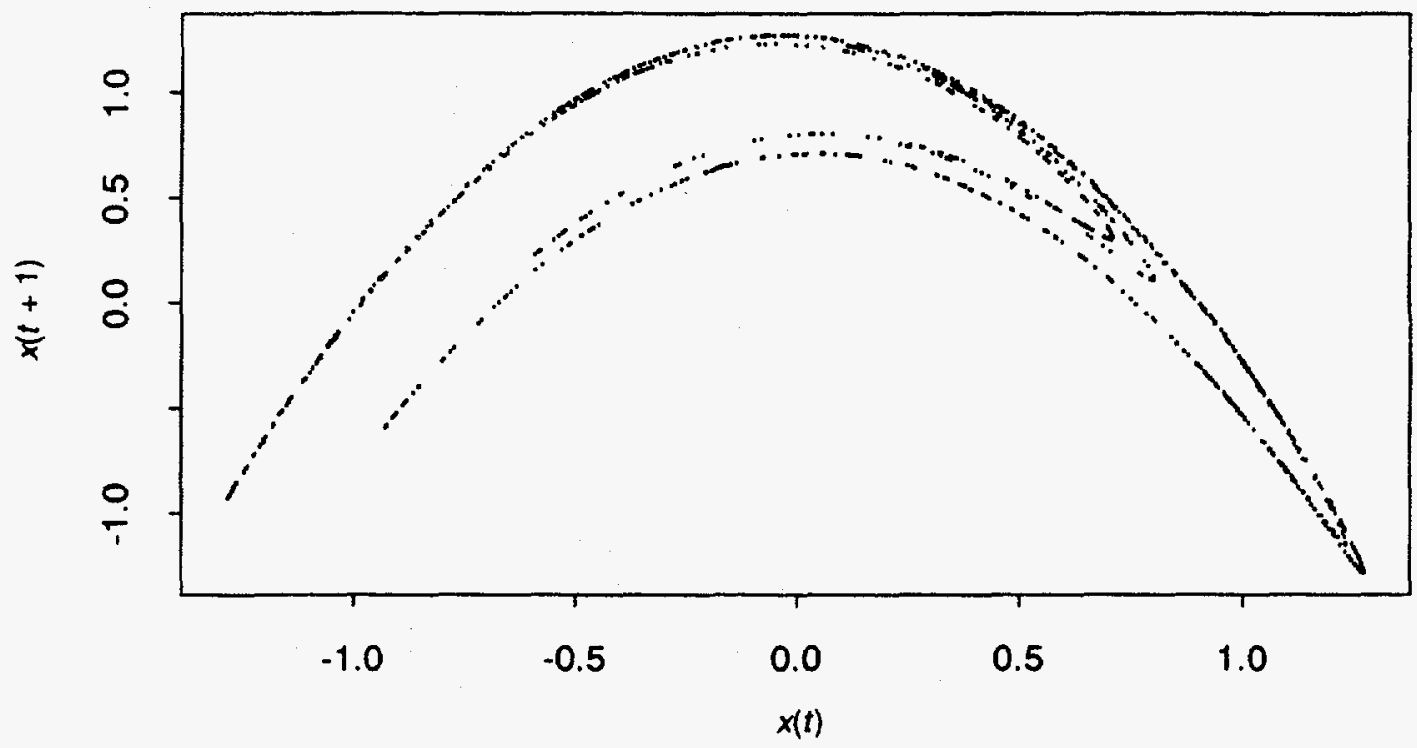

(a)

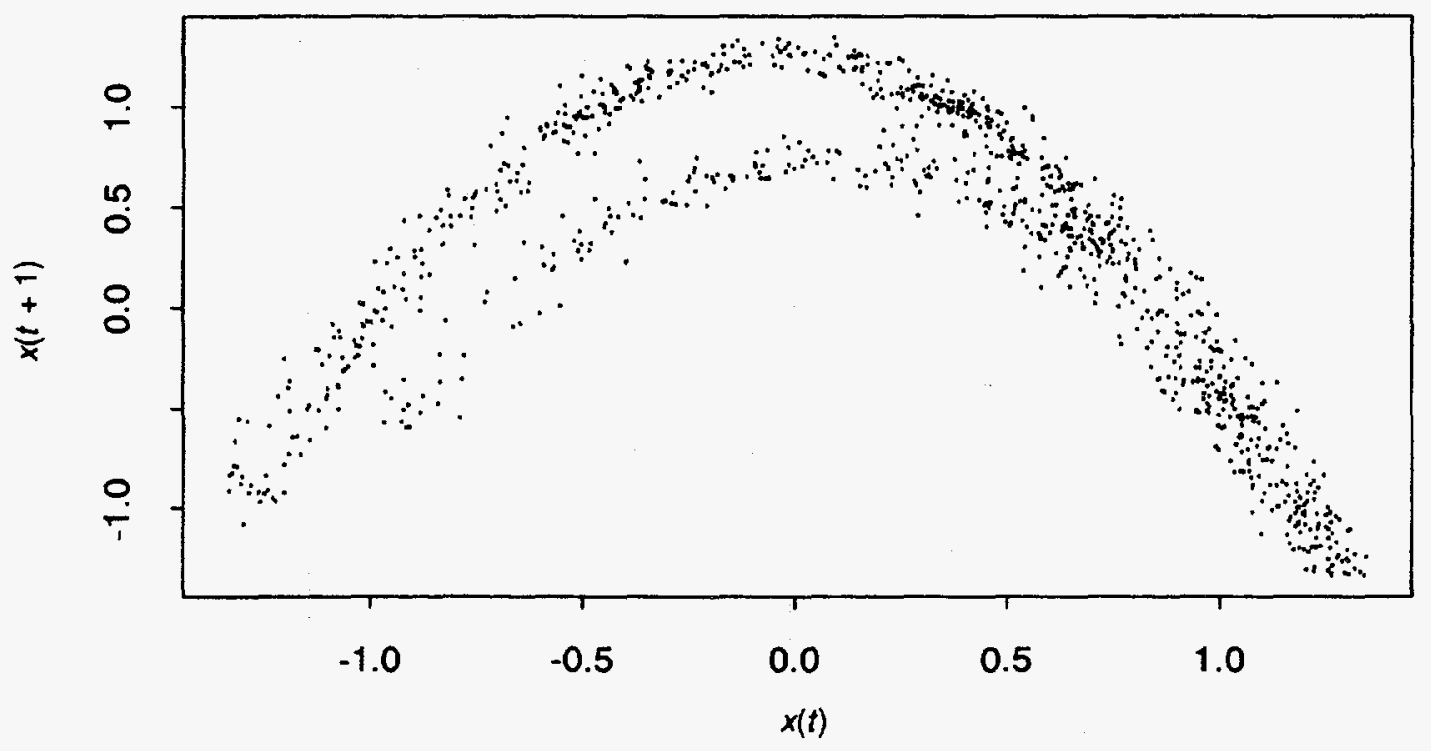

(b)

Fig. 3-18. The Henon map: (a) observed without error and (b) observed with error. 


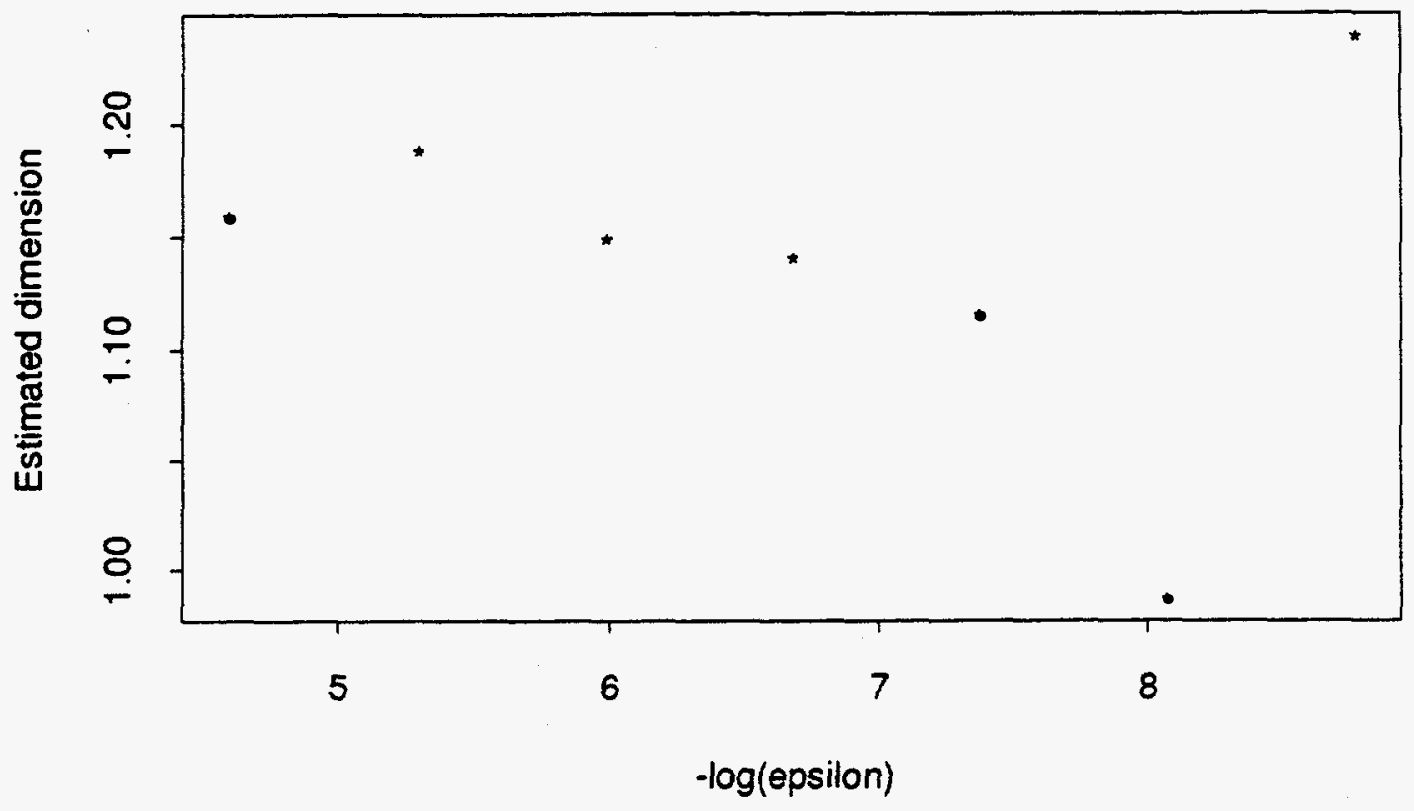

(a)

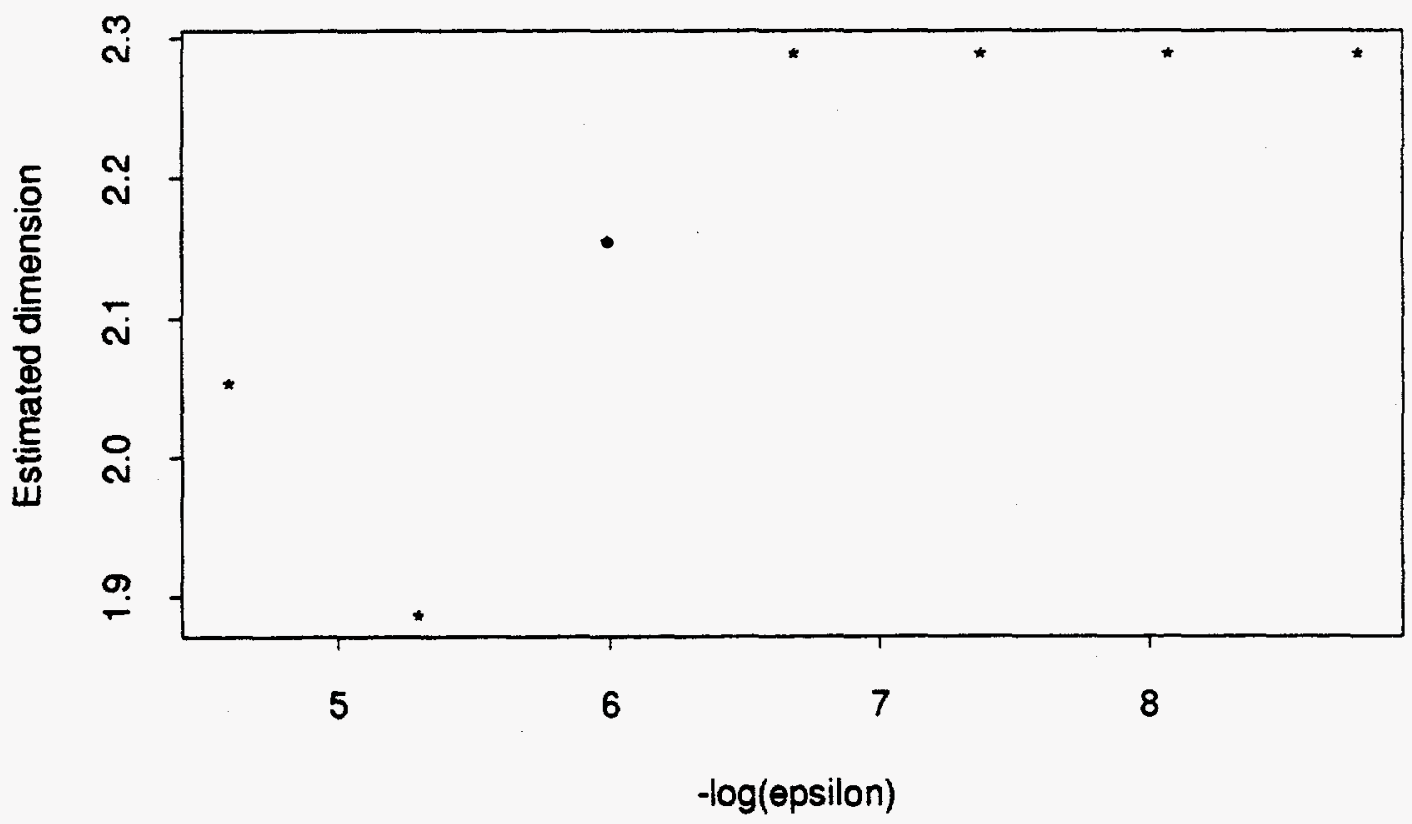

(b)

Fig. 3-19. Dimension estimates of Henon map: (a) observed without error and (b) observed with error. 
map observed both with and without error. We have not yet successfully implemented the procedure suggested in Ref. 3-8 for estimating dimension in the presence of noise. Instead, we applied the same estimation procedure whether the map was observed with noise or not. The true dimension of the Henon map is believed to be approximately 1.2 , so the procedure appears to be working reasonably well in Fig. 3-19a. In Fig. 3-19b, dimension estimates are closer to 2. We do not know what the correct dimension is in this case. We have had less success with estimator 2, so estimation results using estimator 2 are not included here.

To summarize concerning dimension estimates, we have experimented with estimating the dimension of a few maps both with and without noise. We presently believe that the notion of fractal dimension as applied to a time series will have little utility if the time series is observed with error. However, there may be some publications in the near future by Richard Smith that will give more guidance for dimension estimation in the presence of noise.

\section{B . Estimating the Lag $d$}

We mentioned in Sec. II that for the most part we have assumed that the lag is known. Having to estimate the lag complicates matters, but a reasonable method is available. The idea is that the conditional variance $\sigma_{d}^{2}$ of $X_{t}$ given $X_{t-1}, X_{t-2}, \ldots, X_{t-d}$ is nondecreasing in $d$. Loosely, we do no worse in estimating $X_{t}$ if we condition on variables so far back in the past that they do not affect $X_{t}$. The situation is similar to the phenomenon in regression, whereby the residual sum of squares can only decrease when new predictors are added to the model. Therefore, if we estimate $\sigma_{d}^{2}$, for $d=1,2, \ldots, n$, the estimates should decrease up to the true value of $d$ and then remain approximately constant. A cross validation method ${ }^{3-3}$ uses this idea and includes a penalty term for model complexity, which is the size of the proposed lag. We have a FORTRAN program to implement the procedure. Here again, the main difficulty involves the choice of smoothing parameter. We have tested the method on several linear and nonlinear maps and have had moderate success. Details are given in Sec. VI.

\section{A Forecasting Algorithm}

Currently, our best forecasting method is to use the estimated conditional mean (see Sec. IV) to estimate a future observation, given all prior observations. A second forecasting algorithm is described in Ref. 3-15. We have a FORTRAN program to implement the procedure. The idea is to use the $k$ nearest neighbors of each "delay" vector $\left.X_{t}=X_{t}, X_{t-1}, X_{t-d}\right)$ to fit a local linear model to be used to predict $X_{t+T}$, which is $T$ steps ahead, where $T=1,2, \ldots, n$. The first part of the observed time series is used as a fitting set to fit the local linear model. The second part of the time series is used to test by evaluating how well the predictions match the observations. The parameter $k$ is adjustable and if small values of $k$ give good predictions, then 
that is evidence that the time series is deterministic. Otherwise, the series includes stochastic components. We are primarily interested in making good forecasts that help provide a more sensitive test for loss of nuclear material. We mention the relation between the value of $k$ and the size of the stochastic component in the time series because the method was developed as a means to distinguish deterministic from stochastic models. The forecasting algorithm in Ref. $3-15$ is a variation of our conditional mean approach. However, it requires considerably more computation because it uses a weighted least squares fitting procedure for each delay vector in the fitting set. To date, we have not had good success with this method. We include forecasting results using both approaches in Sec. VI.

\section{COMPARISON OF NONLINEAR AND LINEAR PREDICTION RESULTS}

In this section we compare prediction results using nonlinear and linear methods in the following two settings.

1) Compare the mean squared error of prediction (MSEP) using one linear and two nonlinear methods. The MSEP is an indication of how well a procedure can detect loss. The procedure is as follows. Divide the time series vector into a testing set and a training set and assume no loss has occurred. Use the training set to estimate the conditional mean making either no assumption about the functional form of the conditional mean or assuming that the conditional mean is linear. Compare the MSEP evaluated in the test set in the two cases.

2) Compare loss detection sensitivity using one linear and two nonlinear methods. The procedure is similar to procedure (1), but we experiment with particular loss scenarios. The procedure is as follows. Divide the time series vector into a testing set and a training set and assume no loss has occurred in the training set. Use the linear and nonlinear methods to make predictions, and record the prediction error for each observation. Use the estimated error distribution to select thresholds for the individual tests for loss. For various losses in the testing set, compare the detection sensitivities of the linear and nonlinear methods for the three tests described below.

We can consider the simulated time series to be a sequence of $\mathrm{MBs}$, or in particular, a sequence of MUFs (material unaccounted for). We then can refer to the three tests for loss detection that we have experimented with as the ITMUF test, the CUMUF test, and Page's test applied to the ITMUF sequence. Here, ITMUF refers to independently transformed MUF, CUMUF is the cumulative sum of all past MUFs, and Page's test is the same Page's test used in many quality control applications. The ITMUF test is designed to detect a large loss on a 
single balance period. The CUMUF test is designed to detect protracted loss actoss many balance periods. Page's test is a compromise between the ITMUF and CUMUF tests and is designed to be reasonably good at detecting either abrupt loss or protracted loss. For a complete explanation of these three tests, see Ref. 3-7 and the references therein. We use the estimated error distribution to select thresholds for the individual ITMUF test, the CUMUF test, and Page's test, each applied to the residuals. For various losses in the testing set, we compare the detection sensitivity of the three tests for the linear and nonlinear methods.

\section{RESULTS:}

1) Regarding the MSEP, we have analyzed simulated data from the following six maps, each observed with error:

a) Henon map, $x_{t}=1-1.4 x_{t-1}^{2}+0.3 x_{t-2}$;

b) Logistic map, $x_{t}=4 x_{t-1}\left(1-x_{t-1}\right)$;

c) $\mathrm{MA}(2), X_{t}=a_{0}+b_{1} \varepsilon_{t-1}+b_{2} \varepsilon_{t-2}+\varepsilon_{t}$

d) $\operatorname{AR}(2), X_{t}=a_{0}+a_{1} X_{t-1}+a_{2} X_{t-2}+\varepsilon_{t}$;

e) $\mathrm{MA}(1), X_{t}=a_{0}+b_{1} \varepsilon_{t-1}+\varepsilon_{t}$; and

f) $\operatorname{AR}(1), X_{t}=a_{0}+a_{1} X_{t-1}+\varepsilon_{t}$.

We have used notation to describe the MA and AR models that is consistent with the notation in (3-1). Restrictions are assumed to hold on the magnitudes of the MA parameters to ensure invertibility and on the magnitudes of the AR parameters to ensure stationarity. Otherwise, the usual differencing techniques would be applied. We have used lower case for the two nonlinear maps, $a$ and $b$, because we generated the data deterministically and then added observational error. Specifically, we generated the $x_{t}$ 's and then added independent $N\left(0,0.2^{2}\right)$ random variables to represent observational errors.

We used training vectors with 1000 observations and testing vectors with 1000 observations. Of interest is the MSEP in each of the six cases.

The MSEPs for two methods of linear estimation and for three types of nonlinear estimation are shown in Table 3-I for the six cases. We include the sample variance $s^{2}$ for each case because $s^{2}$ would be the MSEP if we simply predicted the sample mean to be the value for each observation. In Table 3-I, the first linear method is denoted Linear1, the second linear method is denoted Linear2, and similarly for the three nonlinear methods. 
Table 3-I: Simulation Results for MSEP

\begin{tabular}{lccccc}
\hline & Linear1 & Linear2 & Nonlinear1 & Nonlinear2 & Nonlinear3 \\
\hline $\mathrm{a}\left(s^{2}=0.53\right)$ & 0.47 & 0.49 & 0.09 & 0.07 & 1.30 \\
$\mathrm{~b}\left(s^{2}=0.14\right)$ & 0.14 & 0.14 & 0.05 & 0.28 & 0.56 \\
$\mathrm{c}\left(s^{2}=1.49\right)$ & 1.08 & 1.25 & 1.47 & 0.88 & 1.85 \\
$\mathrm{~d}\left(s^{2}=1.72\right)$ & 1.17 & 1.06 & 1.11 & 0.49 & 0.77 \\
$\mathrm{e}\left(s^{2}=1.22\right)$ & 1.06 & 1.08 & 1.09 & 0.67 & 1.88 \\
$\mathrm{f}\left(s^{2}=1.38\right)$ & 1.07 & 1.07 & 1.08 & 0.18 & 0.58 \\
\hline \hline
\end{tabular}

The first linear method is to fit the best possible ARMA model to the observed sequence and use the fitted model to forecast. The second linear method fits a linear model to the regression of $X_{t}$ on $X_{t-1}$ or on $X_{t-1}$ and $X_{t-2}$. There is not much difference in MSEP between the two linear methods in cases a-f.

The first nonlinear method is our conditional mean estimator using a nonadaptive kernel and allowing only lag 1 . The second nonlinear method is the "final prediction error" method suggested in Ref. 3-3 in the context of estimating the lag. The best estimate of the lags for models (a) through (f) was: $d=2,2,2,2,2$, and 3, respectively. For an $\operatorname{MA}(p)$ or $\operatorname{AR}(p)$ model, the correct lag is $p$. Therefore, the final prediction error method gave incorrect results for the MA(1) and AR(1) models.

The third nonlinear method is the method described in item 3 of Sec. V. This is a computationally intensive method, which appears to be an inconsistent performer in our experiments to date. For cases a, b, c, and e, this method performed poorly.

In comparing the linear methods to the nonlinear methods, note that cases $a$ and $b$ are the only nonlinear maps. In cases $a$ and $b$ there is a clear advantage in using the first nonlinear method over the linear methods.

Case $\mathrm{c}$ is the MA(2) map, and the first nonlinear method performed poorly. Recall that this method uses only lag one. It might therefore seem surprising that this method did so well on the Henon map, which is lag two. However, the lag one term in the Henon map is the most important contributor to the conditional mean, so this might be expected. With the second nonlinear method, we experimented in all cases with one to five lags. Note that for cases c-f, which are the linear maps, this second nonlinear method performs better than either linear method. We are surprised by this because for truly linear maps we expect linear models to be optimum. We speculate that this performance by the second nonlinear method is because of the large (1000 observations) sample size used. If the sample size is large, there is little lost by not assuming a linear map when the true map is linear. 
2) Regarding loss detection, we have compared the loss detection sensitivity of linear and nonlinear estimation for data simulated according to the function

$$
X_{t}=-\sin \left(X_{t-1}+\varepsilon_{t-1}\right)+\varepsilon_{t}
$$

where $\varepsilon_{q} \sim(0,1)$. The true conditional mean is therefore nonlinear. We may regard Eq. (3-22) as describing how successive MBs are generated when there has been zero loss of material. The functional form in Eq. (3-22) was chosen for illustrative purposes. There was no attempt to model any particular reprocessing plant. We previously considered the map given by Eq. (322) in Figs. 3-5, 3-6, and 3-7, which were referred to in Sec. IV.

We used 10000 observations to estimate the conditional means, both for the linear and the nonlinear methods. We then used 1000 sets of 10 observations to determine the prediction errors, $\varepsilon_{i}=X_{i}-E\left(X_{i} \mid X_{i-1}\right)$. We used the 10 errors per set to select thresholds for alarms, allowing approximately 50 false alarms in the 1000 sets for approximately a type I error probability of 0.05 ; that is, $\alpha=0.05$ using typical statistical notation. For the nonlinear method, we selected the thresholds $h=4$ and $k=0.5$ for Page's test and thresholds of 2.85 and 2.5 for the standardized CUMUF and ITMUF, respectively. The standardized CUMUF is the observed CUMUF divided by its estimated standard deviation and similarly for the standardized ITMUF. For the linear method, we selected the thresholds $h=4.5$ and $k=0.5$ for Page's test and thresholds of 3.0 and 2.2 for the standardized ITMUF test and CUMUF test, respectively. Based on the values of these empirically chosen thresholds, we expect the nonlinear method to detect loss more efficiently than the linear method for Page's and the ITMUF test, and vice versa for the CUMUF test. This is because a Page's test having $k=0.5$ and $h=4$ is more sensitive to loss than a Page's test having $k=0.05$ and $h=4.5$ and similarly for the other two tests. The simulation results given below confirm our expectations.

Because the errors are independent under the assumption of no loss, another possible approach is to use the estimated error variance to calculate thresholds. For example, if the error variance were 1.5 and we performed a one-sided test, the alarm threshold would be $1.65 \times 1.5$ $=2.475$, which would mean that any $\varepsilon_{i}>2.475$ would signal an alarm at the $\alpha=0.05$ level. For 10 independent tests, each individual test would be conducted at the $\alpha=0.05 / 10$ level. In the present context, by type I error probability, we mean the probability of an alarm during the set of 10 observations when there has been no loss of material.

We do not require nearly as many as 10000 observations to apply either estimation technique. A few hundred observations should suffice, but we chose to use 10000 so that any observed performance differences in the two methods were not likely to be due to chance. Also 
we arbitrarily divided the observations into sets of 10 observations because it is current International Atomic Energy Agency (LAEA) practice to require approximately monthly inspections and annual plant cleanouts. Therefore, sequences of about 10 or $12 \mathrm{MBs}$ are typical.

We then generated another 1000 sets of 10 observations having four loss scenarios:

a) abrupt loss of 2.2 in balance period 5;

b) abrupt loss of 4.4 in balance period 5;

c) protracted loss of $2.2 / 3$ in balance periods 5,6 , and 7; and

d) protracted loss of $4.4 / 3$ in balance periods 5, 6, and 7 .

The abrupt loss of 2.2 is twice the estimated standard deviation of the errors from the nonlinear estimation method. Results are shown in Tables 3-II and 3-III. As expected from the size of the thresholds, for Page's test and the ITMUF test, the nonlinear method has a higher detection probability than the linear method and vice versa for the CUMUF test.

\begin{tabular}{lccccc}
\hline \hline Table 3-II. Simulation Results for the Two Abrupt Losses \\
\cline { 2 - 3 } \cline { 5 - 6 } & \multicolumn{2}{c}{ Loss a } & & \multicolumn{2}{c}{ Loss b } \\
\cline { 2 - 3 } \cline { 5 - 6 } & Nonlinear & Linear & & Nonlinear & Linear \\
\hline Page & 212 & 189 & & 736 & 702 \\
ITMUF & 323 & 279 & & 914 & 878 \\
CUMUF & 66 & 107 & & 49 & 112 \\
\hline \hline
\end{tabular}

\section{Table 3-III. Simulation Results for the Protracted Abrupt Losses}

\begin{tabular}{lccccc}
\hline & \multicolumn{2}{c}{ Loss c } & & \multicolumn{2}{c}{ Loss d } \\
\cline { 2 - 3 } \cline { 5 - 6 } & Nonlinear & Linear & & Nonlinear & Linear \\
\hline Page & 218 & 172 & & 679 & 510 \\
ITMUF & 153 & 113 & & 494 & 348 \\
CUMUF & 82116 & 107 & & 193 & \\
\hline \hline
\end{tabular}

We currently have no explanation why the CUMUF test performs better in this example using the linear method than using the nonlinear method. 


\section{SUMMARY AND PLANS FOR FUTURE RESEARCH}

This report has served as a literature review and data analysis.

There are at least two possible nuclear materials safeguards applications for some of the recent advances in nonlinear time series analysis. First, if we had a long sequence of observed MBs, which were known to have mean zero (no true loss), then we could estimate the mean and variance of a given $\mathrm{MB}$ conditional on all prior MBs. It is possible that knowledge of the approximate functional form of these conditional means and variances would improve the loss detection capability.

Second, under the assumption of no true loss, knowledge of the approximate functional form of these conditional means and variances would provide some information about the physical processes generating the observed sequences.

In the short (length 144) MB sequence that we have analyzed, there was no evidence of nonlinearity. One goal is to try some of the techniques that were described in Secs. IV and V on more time series, both real and simulated.

The literature on the analysis of time series is large and growing. We have presented some of the recent nonparametric approaches, with particular emphasis on developing the most sensitive test for loss of nuclear material. Along that line, we propose to compare three candidate methods of monitoring an MB time series. Here is a brief summary of the three methods.

1. The traditional method assumes that fundamental measurement errors can be estimated and then variance propagation can be used to estimate the covariance matrix of the observed MB sequence. A best test for loss of material is available in the sense of the Neyman-Pearson lemma only if the loss scenario is known. Of course, if the loss scenario is known, there is no need for a test. Therefore, for practical purposes, there is no best test available and the safeguards literature contains several simulation studies to compare the performances of various candidate tests under various loss scenarios. We have not reviewed those tests in this report, but propose to use some of the proposed tests for the sake of comparison.

2. A recent approach ${ }^{3-16}$ applied a standard ARMA model to shipper-receiver differences. If we assume a fixed lag, we could apply an ARMA (probably an MA) model to an MB sequence. The estimated ARMA parameters can be used for forecasting as we demonstrated in Sec. VI.

3. The recent advances in nonlinear time series modeling have been described in some detail and several new forecasting methods have been attempted. In Ref. 3-17 we provide additional technical background, as well as simulation results using our conditional mean estimator along with other nonparametric estimates, on both linear 
and nonlinear time series. We have encountered the typical technical difficulty associated with most nonparametric estimation attempts. That is, the choice of smoothing parameter can drastically affect the results. We have added an adaptive smoothing feature to our procedure to estimate the conditional mean. Adaptive smoothing attempts to vary the smoothing parameter according to the local density of the data. Our adaptive smoothing method has sometimes outperformed our non-adaptive smoothing method, but not always. The results in Table 3-I of Sec. VI were from our non-adaptive smoothing implementation of the conditional mean estimator. We have recently learned ${ }^{3-18}$ of bootstrapping methods to help choose the smoothing parameter and believe these methods should be investigated.

Our current emphasis is therefore on prediction, which should provide a sensitive test for loss of nuclear material. Consider a time series $X_{1}, X_{2}, \ldots, n$ and assume that the lag $d=1$. The $X_{t}$ 's might denote an MB sequence. The best estimate (in the sense of minimizing the average squared error) of $X_{t}$ is the conditional mean, $M(x)=\mathrm{E}\left(X_{t} \mid X_{t-1}=x\right)$. It is therefore a reasonable goal to estimate $M(x)$ using a nonparametric estimator such as the one described in Sec. IV. We also point out that the goal in nonlinear modeling might include not only prediction but, in addition, a better understanding of the process that is generating the data. It is therefore reasonable to consider nonparametric estimates of various properties (such as conditional mean and local Lyapunov exponents) of the function generating the data.

\section{REFERENCES:}

3-1. C. Chatfield, The Analysis of Time Series: An Introduction, Second edition (Chapman and Hall, London, 1980).

3-2. L. Mark Berliner, "Likelihood and Bayesian Prediction of Chaotic Systems," JASA 86(416), 938-952 (1991).

3-3. B. Cheng and $\mathrm{H}$. Tong, "On Consistent Nonparametric Order Determination and Chaos," JRSSB 54(2), 427-449 (1992).

3-4. Bjorn Auestad and Dag Tjostheirn, "Identification of nonlinear time series: First order characterization and order determination," Biometrika 77(4), 669-687 (1990).

3-5. Richard Smith, "Estimating Dimension in Noisy Chaotic Time Series," JRSSB 54(2), 329-351 (1992).

3-6. R. R. Picard and J. F. Hafer, "MAWST: Materials Accounting With Sequential Testing, Version 2.0," Los Alamos National Laboratory, Safeguards Systems Group report N-4/91633 (1991). 
3-7. R. R. Picard, "Sequential Analysis of Materials Balances," Nucl. Mater. Manage. XV(2), 38-42 (January 1987).

3-8. T. P. Speed and D. Culpin, "The Role of Statistics in Nuclear Materials Accounting: Issues and Problems," JRSSA A149, Part 4, 281-313 (1986).

3-9. E. Leitner, R. Weh, R. Avenhaus, M. Canty, "Sequential Test Procedures for NearReal-Time Accountancy," Nucl. Mater. Manage. XV(2), 29-34 (January 1987).

3-10. John Jaech, Statistical Methods in Nuclear Material Control, Technical Information Center, US Atomic Energy Commission, 1973.

3-11. Ian McKeague and Mei-Jie Zhang, "Identification of Nonlinear Time Series from First Order Cumulative Characteristics," Florida State University Technical Report No. M-853 (August 1991).

3-12. Rodney Wolff, "Local Lyapunov Exponents: Looking Closely at Chaos," JRSSB 54(2), 353-371 (1992).

3-13. H. Tong, Nonlinear Time Series: A Dynamical System Approach (Clarendon Press, Oxford, 1990).

3-14. J. Theiler, B. Galdrikian, A. Longtin, S. Eubank, and J. Farmer, "Using Surrogate Data to Detect Nonlinearity in Time Series," in Nonlinear Modeling and Forecasting, Martin Casdagli and Stephen Eubank, Ed., Proceedings Vol. XII, Santa Fe Institute Studies in the Sciences of Complexity (Addison-Wesley Publishing Co., 1992), pp. 163-188.

3-15. Martin Casdagli, "Chaos and Deterministic Versus Stochastic Nonlinear Modeling," JRSSB 54(2), 303-328 (1992).

3-16. G. Laszlo, and M. Yousir, "Time Series Analysis of Shipper Receiver Differences," Nucl. Mater. Manage. XXI, 259-263 (1992).

3-17 Tom L. Burr, "Predicting Linear and Nonlinear Time Series with Applications in Nuclear Safeguards and Nonproliferation," Los Alamos National Laboratory report LA12766-MS (April 1994).

3-18. Christian Leger, Dimitris Politis, and Joseph Romano, "Bootstrap Technology and Applications," Technometrics 34(4), 378-398 (1992). 